\title{
From individual spiking neurons to population behavior: Systematic elimination of short-wavelength spatial modes
}

\author{
Moira L. Steyn-Ross* and D. A. Steyn-Ross \\ School of Engineering, University of Waikato, Hamilton, New Zealand \\ (Received 9 August 2015; revised manuscript received 1 December 2015; published 1 February 2016)
}

\begin{abstract}
Mean-field models of the brain approximate spiking dynamics by assuming that each neuron responds to its neighbors via a naive spatial average that neglects local fluctuations and correlations in firing activity. In this paper we address this issue by introducing a rigorous formalism to enable spatial coarse-graining of spiking dynamics, scaling from the microscopic level of a single type 1 (integrator) neuron to a macroscopic assembly of spiking neurons that are interconnected by chemical synapses and nearest-neighbor gap junctions. Spiking behavior at the single-neuron scale $\ell \approx 10 \mu \mathrm{m}$ is described by Wilson's two-variable conductance-based equations [H. R. Wilson, J. Theor. Biol. 200, 375 (1999)], driven by fields of incoming neural activity from neighboring neurons. We map these equations to a coarser spatial resolution of grid length $B \ell$, with $B \gg 1$ being the blocking ratio linking micro and macro scales. Our method systematically eliminates high-frequency (short-wavelength) spatial modes $\vec{q}$ in favor of low-frequency spatial modes $\vec{Q}$ using an adiabatic elimination procedure that has been shown to be equivalent to the path-integral coarse graining applied to renormalization group theory of critical phenomena. This bottom-up neural regridding allows us to track the percolation of synaptic and ion-channel noise from the single neuron up to the scale of macroscopic population-average variables. Anticipated applications of neural regridding include extraction of the current-to-firing-rate transfer function, investigation of fluctuation criticality near phase-transition tipping points, determination of spatial scaling laws for avalanche events, and prediction of the spatial extent of self-organized macrocolumnar structures. As a first-order exemplar of the method, we recover nonlinear corrections for a coarse-grained Wilson spiking neuron embedded in a network of identical diffusively coupled neurons whose chemical synapses have been disabled. Intriguingly, we find that reblocking transforms the original type 1 Wilson integrator into a type 2 resonator whose spike-rate transfer function exhibits abrupt spiking onset with near-vertical takeoff and chaotic dynamics just above threshold.
\end{abstract}

DOI: 10.1103/PhysRevE.93.022402

\section{INTRODUCTION}

Effective modeling of brain dynamics requires assimilation of activity over multiple scales. At the cellular level, a resting neuron emits an action potential, or "spike," when its membrane voltage exceeds a threshold of about $-60 \mathrm{mV}$. The process of spike generation is governed by voltagedependent changes in $\mathrm{Na}^{+}$and $\mathrm{K}^{+}$ion-channel conductances that shape the growth, deceleration, and subsequent recovery of the membrane voltage. Hodgkin and Huxley [1] provided the foundation description for spiking dynamics, and many simplified variants of these deterministic equations (e.g., [2-5]) have been studied. Synaptic and ion-channel noise can have a significant impact on neuron dynamics, and this has stimulated investigation of stochastic adaptations of these model equations using both additive noise and Markov-chain approaches [6-11].

Information processing in the brain is presumed to involve the collective and coordinated activity of large groups of neurons [12]. For example, in the visual cortex, cortical columns containing 100 to 100000 neurons respond to specific orientations of bar-shaped visual stimuli [13]. Similarly, the

\footnotetext{
*msr@waikato.ac.nz
}

Published by the American Physical Society under the terms of the Creative Commons Attribution 3.0 License. Further distribution of this work must maintain attribution to the author(s) and the published article's title, journal citation, and DOI. electrical signal recorded from a $\sim 1 \mathrm{~cm}^{2}$ electroencephalogram (EEG) electrode represents the summed activity of many thousands of neurons. This motivates a pair of questions. How does one develop a model that encapsulates the summed network activity of large populations of spiking neurons? Also, how does noise - both synaptic and ion-channel - percolate up from the micro scale of the single neuron to the macro scale of the network?

One approach is to construct hierarchical networks of individually coupled spiking neurons. The Human Brain Project is attempting to scale up such networks to realistic neuron counts, but this is a massive and complex computational task requiring supercomputing facilities $[14,15]$.

At the other complexity extreme is the top-down mean-field approach that replaces individual neurons with a spatially averaged neural continuum which describes population activity in terms of spike rates, making no attempt to follow the detailed submillisecond dynamics of spike generation and propagation. In this approach, pioneered by Wilson and Cowan [16], the input to each neuron in the network is the weighted sum of input from excitatory and inhibitory neural populations whose activity is set by a sigmoidal function mapping population-average membrane voltage to firing rate. Mean-field models provide a useful depiction of bulk properties of large aggregates of neurons; however, their rate-based foundation imposes an ad hoc spatial and temporal averaging that neglects fluctuations and cannot track the percolation of channel and synaptic noise from the individual neuron up to the population. Despite these limitations, the mean-field formalism has been adopted by many authors to 
describe a range of brain rhythms and state transitions and remains a very active area of cortical modeling [17-22].

Recently, there has been significant interest in moving beyond mean-field theory to provide a more accurate mapping from single spiking neurons to populations [12,13,23], with several authors adopting a master equation formulation [24-28]. The master equation has a rich history in the depiction of jump processes associated with chemical reactions and other birth-death interactions [29]. In simple terms, the master equation follows the time development of the conditional probability distribution function $P\left\{A(t) \mid A^{\prime}(t-\Delta t)\right\}$, where $A(t)$ is the population activity at time $t$, and $A^{\prime}(t-\Delta t)$ is population activity at a slightly earlier time. Using a Markovian approximation, and assuming the system is quasistationary during small time interval $\Delta t$, the probability per unit time that a neuron will spike is governed by a transition operator, $W\left\{A(t) \mid A^{\prime}(t)\right\}$, whose spatial average yields a sigmoidal firing-rate transfer function for the neural population [26]. The master equation is then solved by deriving a set of moment hierarchy equations that are contingent on the properties of the transfer function. The fact that this method relies on a rather ad hoc spatial averaging means that it is not all clear how noise contributions from ion-channel and synaptic sources are to be aggregated all the way up to the scale of the population.

To address these issues, we present a scheme to scale systematically the dynamics of a single spiking neuron to the bulk environment of an assembly of such neurons. We proceed by regridding the cortical system using a spatial blocking (coarse-graining) technique similar to that employed in K. Wilson's renormalization group theory for critical phenomena [30]. In this landmark paper, Wilson transformed the $S^{4}$ model (a representation of the Ising ferromagnet) to momentum space and integrated over high-wave-number (small-spatial-scale) modes using generalized path integrals. The blocking formalism we adopt here utilizes the adiabatic elimination of spatial scales developed by Gardiner [29,31,32] and by Steyn-Ross and Gardiner [33]. In particular, Ref. [33] shows that by projecting out the stochastic variables associated with short wavelengths, one can reproduce Wilson's pathintegral blocking equations for the $S^{4}$ ferromagnet. Our rescaling is expressed in a Fokker-Planck framework, making use of boson annihilation-creation operators drawn from quantum mechanics. This methodology provides a transparent formalism which not only describe the aggregation of neuronal noise to the level of the population, but also elucidates how nonlinear interactions at the micro scale feed into the rescaled population equations.

We start with a two-dimensional (2D) patch of cortical tissue partitioned into a network of $(2 n+1)^{2}$ unit cells, each cell of area $\ell^{2}(\ell \approx 10 \mu \mathrm{m})$ and containing a pair of excitatory $(e)$ and inhibitory $(i)$ neurons; neurons are coupled via both chemical and electrical ( $i-i$ and $e-e$ gap-junction) synapses, and are assumed to be perturbed by synaptic and ion-channel white noises. We adopt the spiking-neuron model of H. R. Wilson [4,5], an elegant encapsulation of the essential dynamics of the standard conductance-based Hodgkin-Huxley (HH) equations. The compelling advantage of the H. R. Wilson model is its ability to reproduce biophysically plausible spiking wave forms for both type 1 (integrator) and type 2 (resonator) excitable tissue using only polynomial (rather than transcendental) nonlinearities in the voltage and recovery differential equations; this reduced model has been used by numerous authors $[8,23,34,35]$.

The 2D patch of cortex is then mapped to a coarser-grained network of $(2 N+1)^{2}$ compartments of side length $L=$ $B \ell$ with $L \approx 1 \mathrm{~mm}$ and where $B=(2 n+1) /(2 N+1) \gg 1$ defines our blocking ratio (see Fig. 4). Each of these larger compartments represents a neural macrocolumn, containing an interconnected population of $\sim 10^{5}$ neurons. In this way, we map the H. R. Wilson single-neuron equations to the areal extent of a macrocolumn, with rescaled equations describing stochastic variables that have been spatially averaged over the coarser grid. Extracting the correct coarse-grained scaling is the essential goal of mean-field neural theory.

As is the case for K. Wilson's path-integral approach, our method is inherently perturbative; in our case we require at least a minimal level of gap-junction diffusivity. This requirement is discussed further in Sec. IV and Appendix F.

In summary, this paper shows how spiking activity and neuronal noise can be systematically reblocked from singleneuron level to neural population. We anticipate that these reblocked equations could be used to (i) derive transfer functions relating membrane voltage to the averaged firing rate of the macrocolumn taking nonlinear neuron-neuron interactions into account; (ii) investigate changes in fluctuation spectra during approach to critical points; (iii) establish scaling laws relating blocking ratio to the temporal and spatial statistics of avalanches in neural activity; (iv) predict the spatial extent of emergent cortical-column structures.

The paper is structured as follows. In Sec. II we describe the embedding of the spiking-neuron model within a continuum neural field of spiking neurons. We detail the properties of the spiking neuron and the nature of the synaptic and diffusive inputs that drive its voltage and recovery system variables. We transform the equations of motion to stochastic differential form by introducing additive white noises representing synaptic and ion-channel stochastic perturbations. We map these Langevin equations to a Fokker-Planck formulation on a finegrained (micro-scale) cortical grid, then apply a systematic and rigorous adiabatic elimination of short-wavelength (high- $\vec{q}$ ) spatial modes to reach our end goal: a rescaled Fokker-Planck equation describing spiking dynamics on a coarse-grained (macro-scale) cortical continuum.

As a preliminary demonstration of our rescaling approach, in Sec. III we investigate the small-noise deterministic limit of a coarse-grained spiking neuron embedded in a network of diffusively coupled neurons whose chemical synapses have been disabled. We conclude in Sec. IV with a discussion of the method, implications, and possible applications. The six appendixes provide technical details describing reblocking corrections, creation and annihilation operators, evaluation of expectation values, Fourier inversion of drift and diffusion terms, wave-number integral definitions, and gap-junction connectivity.

\section{METHODS}

In broad outline, we start with a spiking-neuron model embedded within a continuum field of identical spiking neurons, then develop a systematic mechanism for aggregating 
(a)

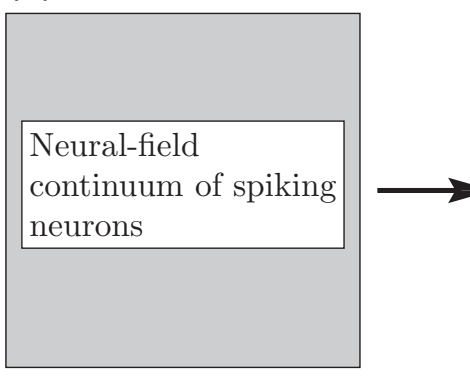

(d)

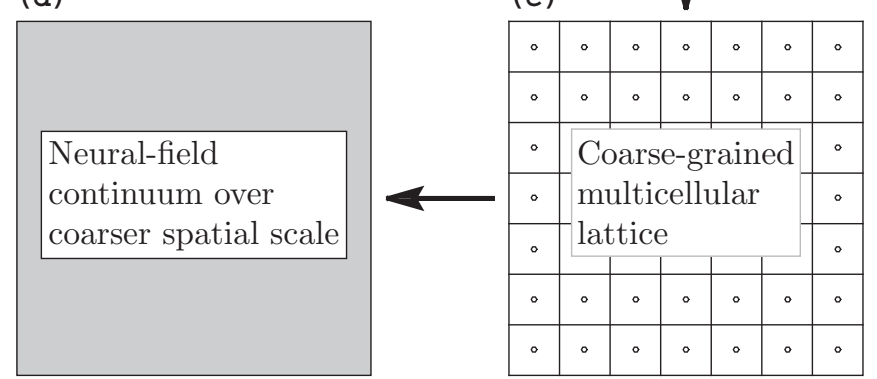

FIG. 1. Mapping of cortical field equations to a coarser spatial scale. (a) Continuum field equations for excitable cortical tissue are discretized to a fine-grained micro-scale lattice of spiking neurons (b) [see Fig. 4(a)], then reblocked onto a coarser spatial grid (c) [Fig. 4(b)] before inversion back to continuum form (d). The result is a set of spiking-neuron field equations describing spatiotemporal dynamics at the coarser spatial scale.

the nonlinear dynamics of a spiking neuron up to a spatial scale encompassing a large population of $\sim 10^{4}$ to $10^{5}$ such neurons coupled via chemical and electrical (gap-junction) synapses.

The major steps are diagramed in Fig. 1.

Itemizing the structure of this Methods section, we construct, then consolidate, the spiking-neuron and field equations in Secs. II A and II B; introduce noise and Langevin forms (Sec. II C); and transform these to a Fokker-Planck description for a fine-grained (micro-scale) lattice of unit cells (Sec. II D). We outline the projector-operator formalism used to eliminate high-wave-number (short-wavelength) spatial modes (Sec. II E); and detail the construction of a rescaled Fokker-Planck equation in which the short-wavelength $\vec{q}$ modes are eliminated in favor of long-wavelength $\vec{Q}$-modes on the coarse-grained (macro-scale) lattice (Sec. II F). This generates a set of rescaled Langevin equations describing spatially averaged spiking dynamics for a macrocolumn (Sec. II G).

\section{A. Model}

\section{Selection of spiking-neuron model}

We seek a simplified spiking-neuron model that is both physiologically meaningful and mathematically tractable. Following Hodgkin and Huxley [1], we ignore the spatially extended dendritic and axonal compartments of a real biophysical neuron and assume that the neuron can be collapsed to an isopotential point. Despite this vast simplification, the
$\mathrm{HH}$ equations are still too complicated for our purpose since they are defined in terms of transcendental (exponential and sigmoidal) voltage dependencies in the gating variables and their time constants, so they cannot be analyzed readily in a perturbative expansion. At the opposite extreme of mathematical complexity is the integrate-and-fire neuron [36], but this model cannot generate action potentials; instead the spike is manually "pasted in" when the membrane voltage crosses an externally specified threshold. Although the popular quadratic integrate-and-fire models, such as those due to Izhikevich [37], can spontaneously fire a spike via a regenerative feedback mechanism, they require a manual reset after firing; thus, their defining equations are inherently nonanalytic and cannot be used here.

For the present work we elect to use the H. R. Wilson twovariable spiking-neuron model [4,5]. Based on the Rinzel [38] simplification of the $\mathrm{HH}$ neuron, the Wilson model replaces transcendental nonlinearities with algebraic polynomial approximations to reproduce the essential action-potential dynamics. Like the FitzHugh-Nagumo (FHN) neuron, the Wilson equations are based on a cubic voltage nonlinearity, but, unlike FHN, emphasize biophysical relevance by retaining explicit reference to $\mathrm{Na}^{+}$and $\mathrm{K}^{+}$reversal potentials. The Wilson model is a reduction of the $\mathrm{HH}$ conductance-based model to a minimal form required for spike generation with fast positive feedback from $\mathrm{Na}^{+}$influx and slower negative feedback from $\mathrm{K}^{+}$outflux. The Wilson equations [5] are expressed in terms of membrane voltage $V$ and a dimensionless recovery variable $R$ representing the rate of $\mathrm{K}^{+}$channel activation,

$$
\begin{gathered}
C \frac{d V}{d t}=-m_{\infty}(V)\left(V-E_{\mathrm{Na}}\right)-g_{\mathrm{K}} R\left(V-E_{\mathrm{K}}\right)+I^{\mathrm{ext}}, \\
\tau \frac{d R}{d t}=-R+R_{\infty}(V) .
\end{gathered}
$$

The first equation describes changes in membrane potential $V$ in terms of membrane specific capacitance $C$, ionic currents due to sodium (first term) and potassium (second term), and external stimulating current $I^{\text {ext }} . E_{\mathrm{Na}}$ and $E_{\mathrm{K}}$ are the equilibrium potentials for $\mathrm{Na}^{+}$and $\mathrm{K}^{+}$, respectively; $m_{\infty}(V)$ is the activation function defining $\mathrm{Na}^{+}$conductance, while the product $g_{\mathrm{K}} R$ represents the recovery-dependent modulation of $\mathrm{K}^{+}$conductance $g_{\mathrm{K}}$. In the second equation, the rate change of recovery $R$ depends on its voltage-dependent steady state $R_{\infty}(V)$ and time constant $\tau$.

The $m_{\infty}(V)$ and $R_{\infty}\left(V_{b}\right)$ are quadratic functions of membrane voltage defined by

$$
\begin{gathered}
m_{\infty}(V) / C=a_{0}+a_{1} V+a_{2} V^{2}, \\
R_{\infty}(V)=b_{0}+b_{1} V+b_{2} V^{2} .
\end{gathered}
$$

There is no explicit representation of the chloride leakage current in Eq. (1). This is because the leakage current has been absorbed into the linear terms of the $m_{\infty}(V)$ and $R_{\infty}(V)$ polynomials [5]. Table I lists the coefficient values; note that we follow the SI-units conventions adopted by M. T. Wilson et al. [23] in their implementation of the H. R. Wilson neuron [5]. 
TABLE I. Model constants for Wilson spiking neuron.

\begin{tabular}{lcc}
\hline \hline Symbol & Value & Unit \\
\hline$C$ & 0.010 & $\mathrm{~F} \mathrm{~m}^{-2}$ \\
$\tau_{b}$ & $5.6 \times 10^{-3}$ & $\mathrm{~s}$ \\
$g_{R, b}$ & $26 \times 10^{3}$ & $\mathrm{~s}^{-1}$ \\
$E_{\mathrm{Na}}$ & $48 \times 10^{-3}$ & $\mathrm{~V}$ \\
$E_{\mathrm{K}}$ & $-95 \times 10^{-3}$ & $\mathrm{~V}$ \\
$a_{0}$ & $17.81 \times 10^{3}$ & $\mathrm{~s}^{-1}$ \\
$a_{1}$ & $475.8 \times 10^{3}$ & $\mathrm{~s}^{-1} \mathrm{~V}^{-1}$ \\
$a_{2}$ & $3.380 \times 10^{6}$ & $\mathrm{~s}^{-1} \mathrm{~V}^{-2}$ \\
$b_{0}$ & 1.26652 & - \\
$b_{1}$ & 37.98 & $\mathrm{~V}^{-1}$ \\
$b_{2}$ & 330 & $\mathrm{~V}^{-2}$ \\
\hline \hline
\end{tabular}

\section{Wilson spiking neuron with diffusion}

For notational convenience, we divide the displacement current density equation (1) by specific capacitance $C$ to give a voltage-update equation, with each term now carrying units of volts per second,

$$
\frac{d V_{b}}{d t}=-g\left(V_{b}\right)\left(V_{b}-E_{\mathrm{Na}}\right)-g_{R, b} R_{b}\left(V_{b}-E_{\mathrm{K}}\right)+I_{b}^{\mathrm{ext}} / C,
$$

where we have introduced subscript $b \in\{e, i\}$ to label the neuron as either excitatory $(e)$ or inhibitory $(i)$. Here $g\left(V_{b}\right) \equiv$ $m_{\infty}\left(V_{b}\right) / C$ and $g_{R, b} \equiv g_{\mathrm{K}} / C$ are the scaled conductances carrying units of inverse seconds (Table I).

We assume that neurons are coupled both diffusively (via direct gap-junction connections) and synaptically (via chemical synapses). Thus, the $I_{b}^{\text {ext }}$ externally sourced current density (amperes per square meter) in Eq. (5) is the sum of incoming diffusive and synaptic currents from other neurons, plus any experimentally imposed control current,

$$
I_{b}^{\mathrm{ext}} / C=\left(I_{b}^{\mathrm{diff}}+I_{b}^{\mathrm{syn}}+I_{b}^{\mathrm{dc}}\right) / C,
$$

which can be expressed as rates of voltage perturbation from diffusive, synaptic, and control sources,

$$
\dot{V}_{b}^{\text {ext }}=\dot{V}_{b}^{\text {diff }}+\dot{V}_{b}^{\text {syn }}+\frac{1}{C} I_{b}^{\mathrm{dc}} .
$$

Diffusion effects also enter the recovery equation (2) as an additive perturbation $R_{b}^{\text {diff }}$ (dimensionless),

$$
\tau_{b} \frac{d R_{b}}{d t}=-\left[R_{b}-R_{\infty}\left(V_{b}\right)\right]+R_{b}^{\mathrm{diff}},
$$

caused by gap-junction mediated changes in intracellular $\mathrm{K}^{+}$ concentration; we discuss the form and construction of this term in Sec. IIA5.

We now describe separately the synaptic and diffusive input terms.

\section{Synaptic input}

The chemical synapses of neuron $b$ (which can be either excitatory or inhibitory) receive an intermittent flux of pulsatile input from action potentials generated by synaptically coupled upstream neuron $a$ (also either excitatory or inhibitory). As illustrated in Fig. 2, we model the transformation from spike activity in presynaptic neuron $a$ to voltage response in the

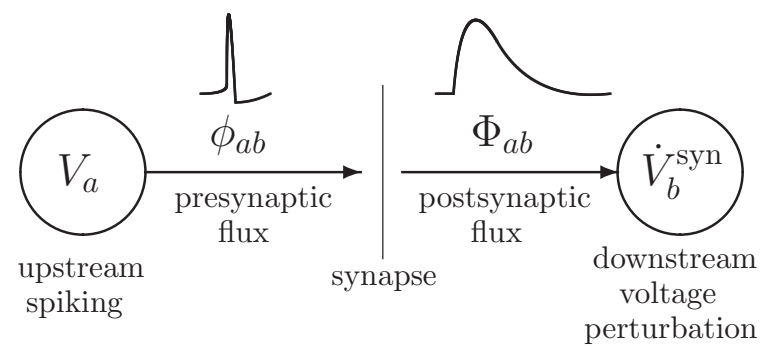

FIG. 2. Spiking activity in upstream neuron $a$ leads to perturbation in the soma voltage of downstream neuron $b$. The flow of activity is mediated by presynaptic flux $\phi_{a b}$ which transforms to postsynaptic flux $\Phi_{a b}$ on crossing the synaptic interface between transmitting axon and receiving dendrite.

soma of postsynaptic neuron $b$ as a three-step process that we summarize as follows.

(1) Voltage spikes generated by source neuron $a$ are conducted along its axon to the synaptic junction with neuron $b$ via a wave equation in $\phi_{a b}(\vec{r}, t)$, the spike flux from $a$ to $b ; \vec{r}$ is the 2D position vector for neuron $b$.

(2) Each spike arriving at neuron $b$ elicits a temporally smoothed and broadened dendritic response $\Phi_{a b}(\vec{r}, t)$ that we model here as biexponential with rise rate $\beta$ and decay rate $\alpha$; the magnitude of the dendritic response is controlled by the deviation of the moving-average soma voltage $\bar{V}_{b}$ from a synaptic reversal potential.

(3) The resulting rate of voltage perturbation at neuron $b$ from chemical-synaptic input is obtained by scaling the dendritic response $\Phi_{a b}$ by the synaptic gain $\rho_{a b}$ (with units volts per spike): $\dot{V}_{b}^{\mathrm{syn}}(\vec{r}, t)=\rho_{a b} \Phi_{a b}(\vec{r}, t)$.

We now elucidate these three stages in more detail.

(i) To represent the axonal propagation of spiking activity $\phi_{a b}$ across a 2D cortical continuum of gray matter, we adopt the wave equation of Robinson et al. [18], which describes damped waves propagating with phase velocity $v$,

$$
\begin{gathered}
{\left[\left(\frac{\partial}{\partial t}+v \Lambda_{a b}\right)^{2}-(v \vec{\nabla})^{2}\right] \phi_{a b}(\vec{r}, t)} \\
=\left(v \Lambda_{a b}\right)^{2} S_{a}\left[V_{a}(\vec{r}, t)-V_{a}^{\mathrm{rest}}\right] .
\end{gathered}
$$

As shown in [18], this equation owns a Macdonald (modified Bessel) connectivity kernel that decays smoothly from a logarithmic singularity at the origin to an asymptotically exponential decay at larger ranges. Thus, the synaptic connectivity pattern is radially symmetric with an approximately exponential axonal length scale of $1 / \Lambda_{a b}$. While we expect that reblocking will tend to filter out intensely local connectivity effects in favor of medium-range-but still local-connections, we make no attempt here to include the nonlocal point-to-point white-matter connections from, e.g., corpus callosum or other cortical structures.

In Eq. (9) we have followed Robinson and Kim [35] and Wilson et al. [23] by introducing a spike normalization $S_{a}$ equal to the inverse area of a prototypical action potential generated by the H. R. Wilson neuron of Eqs (1) and (2),

$$
S_{a}^{-1}=\int_{\text {spike }}\left[V_{a}(t)-V_{a}^{\mathrm{rest}}\right] d t,
$$


with $V_{a}^{\text {rest }}$ being the resting neuron voltage in the absence of external current, $I_{a}^{\text {ext }}=0$. The source term $\left(V_{a}-V_{a}^{\text {rest }}\right)$ in Eq. (9) is only significant while the neuron is spiking.

(ii) The incoming spike flux $\phi_{a b}$ impinges on neuron $b$ to generate a broadened postsynaptic flux $\Phi_{a b}$ that obeys the differential equation

$$
\begin{array}{r}
\left(\frac{d}{d t}+\alpha_{a b}\right)\left(\frac{d}{d t}+\beta_{a b}\right) \Phi_{a b}(\vec{r}, t) \\
=\alpha_{a b} \beta_{a b} \psi_{a b}\left(\bar{V}_{b}\right) N_{a b} \phi_{a b}(\vec{r}, t),
\end{array}
$$

where we have introduced $N_{a b}$, the mean number of synaptic connections from neurons of population $a$ onto each neuron of population $b$, since multiple neurons of population $a$ contribute the field $\phi_{a b}$ [35]. Thus, we may now treat the labels $\{a, b\} \in$ $\{e, i\}$ as identifying excitatory $(e)$ or inhibitory $(i)$ populations of neurons rather than identifying individual neurons.

The biexponential response implied by Eq. (11) approximates the lumped effect of slow ion-channel kinetics for AMPA ( $\alpha$-amino-3-hydroxy-5-methyl-4-isoxazolepropionic acid) or GABA ( $\gamma$-aminobutyric acid) receptors at the postsynaptic membrane [39], and ignores potential saturation effects at high flux rates.

The total incoming flux $N_{a b} \phi_{a b}$ is modulated by $\psi_{a b}$, the postsynaptic reversal-potential function,

$$
\psi_{a b}\left(\bar{V}_{b}\right) \equiv \frac{V_{a}^{\mathrm{rev}}-\bar{V}_{b}(t)}{V_{a}^{\mathrm{rev}}-V_{b}^{\mathrm{rest}}}
$$

which is normalized to unity when the average membrane voltage $\bar{V}_{b}$ matches the zero-current resting voltage [40]. The bar denotes an exponential moving average over a characteristic time scale $T$ that is long compared to the duration of a single spike,

$$
\bar{V}_{b}(t)=-\frac{1}{T} \int_{0}^{t} e^{-\left(t-t^{\prime}\right) / T} V_{b}\left(t^{\prime}\right) d t^{\prime},
$$

which is equivalent to the differential form [35],

$$
\frac{d \bar{V}_{b}(t)}{d t}=-\frac{1}{T}\left[\bar{V}_{b}(t)-V_{b}(t)\right] .
$$

We choose to work with the differential form in our model.

(iii) Incoming postsynaptic fluxes $\Phi_{e b}, \Phi_{i b}$ from Eq. (11) induce voltage perturbations per unit time in population $b$ at position $\vec{r}$ and time $t$ given by

$$
\dot{V}_{b}^{\mathrm{syn}}(\vec{r}, t)=\rho_{e b} \Phi_{e b}(\vec{r}, t)+\rho_{i b} \Phi_{i b}(\vec{r}, t), \quad b \in\{e, i\},
$$

where $\rho_{e b}, \rho_{i b}$ are the synaptic gains with $\rho_{e b}>0$ (excitation) and $\rho_{i b}<0$ (inhibition). The voltage perturbation in downstream population $b$ (which can be either excitatory or inhibitory) is determined by the signed summation of the incoming flux from upstream pools of (excitatory or inhibitory) neurons.

In summary, the firing activity in presynaptic neural population $a$ produces a downstream voltage perturbation in postsynaptic population $b$ via pre- and postsynaptic flux fields $\phi_{a b}$ and $\Phi_{a b}$. This is represented in the flux flow diagram of Fig. 2.

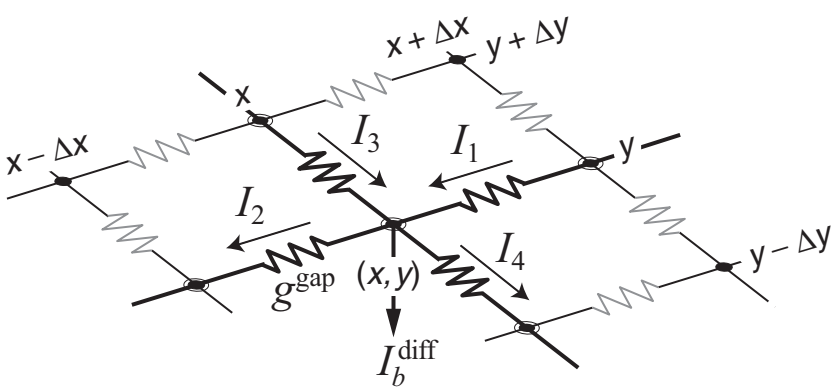

FIG. 3. Cytoplasmic potassium-ion currents flow between cell interiors via open gap-junction connections whose conductance is $g^{\text {gap }} . \mathrm{K}^{+}$will accumulate at the $b$ th neuron at $(x, y)$ if the incoming ionic flux $\left(I_{1}+I_{3}\right)$ exceeds the outgoing flux $\left(I_{2}+I_{4}\right)$, leading to a local increase in potassium concentration, boosting recovery $R_{b}$ by raising transmembrane $\mathrm{K}^{+}$-channel conductance and hence $\mathrm{K}^{+}$efflux into the extracellular space during spiking. Spatial variability in $I_{b}^{\text {diff }}$ will drive diffusion in the recovery variable, i.e., $R_{b}^{\text {diff }} \sim \nabla^{2} I_{b}^{\text {diff }}$.

\section{Diffusive input for membrane voltage}

In addition to pulsatile synaptic flux entering the right-hand side of the $V_{b}$ voltage equation (1), there is also a continuous diffusive current exchange with gap-junction-connected nearest neighbors resulting in perturbation $\dot{V}_{b}^{\text {diff }}$ in the rate change of $V_{b}$ soma voltage, and a corresponding perturbation $R_{b}^{\text {diff }}$ in the recovery equation (2). (The latter is discussed in the next section.)

The presence of such diffusive terms in the voltage and recovery variables is essential for the spatial coarse-graining procedure we adopt in this paper. This is because, as shown in Sec. II E, we eliminate the short-wavelength (large wave number $\vec{q}$ ) modes using a perturbative method based on projection operators which can only converge with appropriate choices for the spatial dependence of the system variables.

Following Steyn-Ross et al. [41], we represent the diffusively coupled network of neurons as a $2 \mathrm{D}$ square lattice of cells, each cell joined to its four nearest neighbors via a gap-junction areal conductance $g^{\text {gap }}$ (Fig. 3). The membrane voltage at node $(x, y)$ is $V_{x, y}$. The total diffusive current per unit area entering the neuron at the $(x, y)$ node is given by summing the $x$ - and $y$-branch residuals, $I^{\text {diff }}=\Delta I_{x}+\Delta I_{y}$, and applying Ohm's law $I=g V$,

$\Delta I_{x}=I_{1}-I_{2}=g^{\mathrm{gap}}\left(\left[V_{x+\Delta x, y}-V_{x, y}\right]-\left[V_{x, y}-V_{x-\Delta x, y}\right]\right)$,

giving

$$
\begin{aligned}
\Delta I_{x} & =g^{\text {gap }}(\Delta x)^{2}\left[\frac{V_{x+\Delta x, y}-2 V_{x, y}+V_{x-\Delta x, y}}{(\Delta x)^{2}}\right] \\
& \approx g^{\text {gap }}(\Delta x)^{2} \frac{\partial^{2} V}{\partial x^{2}} .
\end{aligned}
$$

Similarly, the $y$-branch residual current into node $(x, y)$ is

$$
\begin{aligned}
\Delta I_{y} & =I_{3}-I_{4} \\
& =g^{\text {gap }}(\Delta y)^{2}\left[\frac{V_{x, y+\Delta y}-2 V_{x, y}+V_{x, y-\Delta y}}{(\Delta y)^{2}}\right] \\
& \approx g^{\text {gap }}(\Delta y)^{2} \frac{\partial^{2} V}{\partial y^{2}} .
\end{aligned}
$$


Summing Eqs (16a) and (16b) gives the total mesh current density entering neuron $b$ at $(x, y)$,

$$
I^{\text {diff }} \approx g^{\text {gap }} \ell^{2}\left[\frac{\partial^{2} V}{\partial x^{2}}+\frac{\partial^{2} V}{\partial y^{2}}\right] \equiv g^{\text {gap }} \ell^{2} \nabla^{2} V,
$$

where $\nabla^{2} \equiv \frac{\partial^{2}}{\partial x^{2}}+\frac{\partial^{2}}{\partial y^{2}}$ is the Laplacian operator in twodimensional Cartesian space and where we have written $(\Delta x)^{2}=(\Delta y)^{2}=\ell^{2}$, the area of a square lattice cell of side length $\ell$. Dividing through by the areal capacitance $C$, and assigning label $b$ and position $\vec{r}$ gives us an expression for the diffusion-induced voltage perturbation per unit time,

$$
\dot{V}_{b}^{\operatorname{diff}}(\vec{r}, t)=\frac{g^{\text {gap }} \ell^{2}}{C} \nabla^{2} V_{b}(\vec{r}, t)=D_{b} \nabla^{2} V_{b}(\vec{r}, t),
$$

which defines the voltage-diffusion coefficient $D_{b}$ with SI units $\mathrm{m}^{2} \mathrm{~s}^{-1}$. This is the companion equation to (15) and completes the definitions of the synaptic and diffusive terms on the righthand side (RHS) of (5).

\section{Diffusive input for recovery}

We now invoke a plausibility argument to establish the form of the $R_{b}^{\text {diff }}$ recovery-diffusion term on the RHS of Eq. (2).

The recovery variable $R_{b}$ encapsulates the dynamics associated with delayed opening of potassium ion channels (and inactivation of sodium ion channels) during the middle phases of action-potential generation. The net effect of $\mathrm{K}^{+}$ release (and $\mathrm{Na}^{+}$restriction) is to limit, and then terminate, the action-potential spike $[4,38]$. We focus exclusively on the potassium contribution to the recovery dynamics, since the strongly dominant intracellular ionic species within biological cells is $\mathrm{K}^{+}$(being an order of magnitude more abundant than $\mathrm{Na}^{+}$for mammalian neurons; e.g., see Table 2.4 of [42]); thus, intracellular ionic flows are principally composed of $\mathrm{K}^{+}$ions.

Patch-clamp electrophysiological studies have demonstrated that the single-channel conductance of-and consequent outward current flow through-potassium-ion channels increases with increasing concentrations of intracellular $\mathrm{K}^{+}[43,44]$. Very recently, Andreucci et al. [45] were able to reproduce the current-voltage curves for the $\mathrm{K}^{+}$channel using a theoretical diffusive lattice model, confirming that the level of intracellular $\mathrm{K}^{+}$plays a crucial role in controlling outward current in the $\mathrm{K}^{+}$channel.

Referring to Fig. 3, suppose that for neuron $b$, the gapjunction mediated influx of $\mathrm{K}^{+}$(from neighboring neurons) exceeds the diffusive outflux (to neighboring neurons), resulting in a net inward diffusion $I_{b}^{\text {diff }}$. This will raise the intracellular $\mathrm{K}^{+}$concentration at neuron $b$, enhancing the intensity of the subsequent recovery (voltage-triggered $\mathrm{K}^{+}$release) during the late stages of the next spike event. We argue that $R_{b}^{\text {diff }}$, the diffusive-recovery component of Eq. (2), will be proportional to the two-dimensional spatial variability of $I_{b}^{\text {diff }}$, i.e.,

$$
R_{b}^{\text {diff }} \propto \nabla^{2} I_{b}^{\text {diff }},
$$

which, from Eq. (17), implies a biharmonic (Laplaciansquared) dependence on membrane voltage,

$$
R_{b}^{\text {diff }} \propto \nabla^{2}\left(\nabla^{2} V_{b}\right)
$$

leading to our final form for the diffusive-recovery term as

$$
R_{b}^{\text {diff }}=D_{R} \nabla^{4} V_{b},
$$

where we have introduced a recovery diffusion coefficient $D_{R}$ with SI units $\mathrm{m}^{4} \mathrm{~V}^{-1}$.

\section{B. Consolidated model equations}

For convenient reference we now gather the five differential equations describing a spiking neuron embedded within its neural field of diffusive and synaptic input fluxes. Combining Eqs. (5), (7), (15), and (18) gives the instantaneous membrane voltage,

$$
\begin{aligned}
\frac{\partial V_{b}}{\partial t}= & -g\left(V_{b}\right)\left(V_{b}-E_{\mathrm{Na}}\right)-g_{R, b} R_{b}\left(V_{b}-E_{\mathrm{K}}\right) \\
& +D_{b} \nabla^{2} V_{b}+\sum_{a} \rho_{a b} \Phi_{a b}+I_{b}^{\mathrm{dc}} / C .
\end{aligned}
$$

Meanwhile, incorporating the diffusive-recovery term (19) into recovery equation (2) gives

$$
\frac{\partial R_{b}}{\partial t}=-\frac{1}{\tau_{b}}\left[R_{b}-R_{\infty}\left(V_{b}\right)\right]+\frac{D_{R} \nabla^{4} V_{b}}{\tau_{b}} .
$$

Equation (11) defines the postsynaptic dendritic response $\Phi_{a b}$ arising from an incoming flux $\phi_{a b}$ of presynaptic spiking events,

$$
\left(\frac{d}{d t}+\alpha_{a b}\right)\left(\frac{d}{d t}+\beta_{a b}\right) \Phi_{a b}=\alpha_{a b} \beta_{a b} \psi_{a b}\left(\bar{V}_{b}\right) N_{a b} \phi_{a b},
$$

where $\psi_{a b}$ is the synaptic reversal-potential function (12), operating on $\bar{V}_{b}$, the temporally smoothed membrane voltage from Eq. (14),

$$
\frac{d \bar{V}_{b}(t)}{d t}=-\frac{1}{T}\left[\bar{V}_{b}(t)-V_{b}(t)\right]
$$

and where spike flux $\phi_{a b}$ propagates via the damped wave equation (9),

$$
\left[\left(\frac{\partial}{\partial t}+v \Lambda_{a b}\right)^{2}-(v \vec{\nabla})^{2}\right] \phi_{a b}=\left(v \Lambda_{a b}\right)^{2} S_{a}\left(V_{a}-V_{a}^{\text {rest }}\right),
$$

with spike area normalization $S_{a}$ defined in Eq. (10).

Equations (20)-(24) summarize our construction of a continuum of deterministic spiking neurons.

\section{Stochastic differential equations}

We now introduce low-level white noises $\sqrt{\Gamma} \xi(\vec{r}, t)$ into the voltage, recovery, and flux equations to approximate the biological reality of noisiness in membrane voltage, ion-channel conductance, and synaptic response, respectively. These spatiotemporal white noises have zero mean, are $\delta$ correlated in both time and space,

$$
\begin{aligned}
\langle\xi(\vec{r}, t)\rangle & =0, \\
\left\langle\xi(\vec{r}, t) \xi\left(\vec{r}^{\prime}, t^{\prime}\right)\right\rangle & =\delta\left(\vec{r}-\vec{r}^{\prime}\right) \delta\left(t-t^{\prime}\right),
\end{aligned}
$$

and have intensity $\Gamma$.

After inserting noisy perturbations into the $\left(V_{b}, R_{b}, \Phi_{a b}\right.$, $\left.\phi_{a b}\right)$ system variables, the latter two via their ancillaries $Z_{a b}$ 
and $\Pi_{a b}$, the stochastic versions of Eqs. (20)-(24) read as follows:

$$
\begin{aligned}
\frac{\partial V_{b}}{\partial t}= & \operatorname{RHS}(20)+\sqrt{\Gamma_{b}} \xi_{b}(\vec{r}, t), \\
\frac{\partial R_{b}}{\partial t}= & \operatorname{RHS}(21)+\sqrt{\Gamma_{R_{b}}} \xi_{R_{b}}(\vec{r}, t), \\
\frac{d \Phi_{a b}}{d t}= & Z_{a b}, \\
\frac{d Z_{a b}}{d t}= & -\left(\alpha_{a b}+\beta_{a b}\right) Z_{a b}-\alpha_{a b} \beta_{a b} \Phi_{a b} \\
& +\alpha_{a b} \beta_{a b} N_{a b} \psi_{a b}\left(\bar{V}_{b}\right) \phi_{a b}+\sqrt{\Gamma_{Z_{a b}}} \xi_{Z_{a b}}(\vec{r}, t), \\
\frac{d \bar{V}_{b}}{d t}= & \operatorname{RHS}(23), \\
\frac{d \phi_{a b}}{d t}= & \Pi_{a b}, \\
\frac{\partial \Pi_{a b}}{\partial t}= & -2 v \Lambda_{a b} \Pi_{a b}-\left[\left(v \Lambda_{a b}\right)^{2}-(v \nabla)^{2}\right] \phi_{a b} \\
& +\left(v \Lambda_{a b}\right)^{2} S_{a}\left(V_{a}-V_{a}^{\mathrm{rest}}\right)+\sqrt{\Gamma_{\Pi_{a b}}} \xi_{\Pi_{a b}}(\vec{r}, t),
\end{aligned}
$$

where we have rewritten the second-order post- and presynaptic flux equations (22) and (24) as pairs of coupled first-order differential equations in $\left(\Phi_{a b}, Z_{a b}\right)$ and $\left(\phi_{a b}, \Pi_{a b}\right)$, respectively. Note that we do not insert any additional noise into the slow-soma voltage $\bar{V}_{b}$, so system equation (29) remains deterministic.

The $\xi_{b}(\vec{r}, t)$ additive spatiotemporal white noise in $V_{b}$ [in Eq. (25)] will indirectly induce $\mathrm{Na}^{+}$ion-channel fluctuations [via conductance $g_{b}\left(V_{b}\right)$ ] and gap-junction diffusive fluctuations (via the $\nabla^{2} V_{b}$ Laplacian), while recovery noise $\xi_{R_{b}}(\vec{r}, t)$ in (26) will generate fluctuations in $\mathrm{K}^{+}$ion-channel currents. Although ion-channel noise is usually modeled using discrete
Markov-chain state transitions [9], provided the number of ion channels is sufficiently large, channel-fluctuation statistics can be accurately simulated using appropriately scaled additive white noises [11]. The $\xi_{Z_{a b}}(\vec{r}, t)$ and $\xi_{\Pi_{a b}}(\vec{r}, t)$ noises in the flux (28) and wave (31) equations are a representation of synaptic noise resulting from the continuous background bombardment of activity resulting from the random quantal release of neurotransmitters into the synaptic cleft, evoking miniature postsynaptic currents in the absence of presynaptic spikes [46].

We note that because the regridding and elimination of high- $\vec{q}$ modes is done on the time scale of the fast-soma spiking events, the slow-soma temporal-average voltage $\bar{V}_{b}$ can be treated as a constant, so it does not contribute to the dynamics of the Fokker-Planck equation described below.

\section{Fokker-Planck equation: Unit-cell model}

We consider a square patch of cortical tissue of area $L_{0} \times L_{0}$, with $L_{0} \sim 1 \mathrm{~cm}$ corresponding to the approximate extent of a scalp electrode. As shown in Fig. 4(a), we divide the cortical patch into $(2 n+1)^{2}$ identical square "microcells," each of area $\ell^{2}$, with $\ell \sim 10 \mu \mathrm{m}$. (These microcells will subsequently be aggregated into "macrocells" of linear extent $L \sim 1 \mathrm{~mm}$, corresponding to the approximate scale of a cortical macrocolumn.)

Each microcell is assumed to contain one excitatory and one inhibitory neuron. To compensate for the fact that excitatory neurons are about four times more abundant than inhibitory neurons, an appropriate downscaling of inhibitory chemicalsynaptic connections would be applied.

This simple linear adjustment is expected to be valid for low to moderate levels of neural activity, but is likely to fail at extreme activity levels when nonlinear effects (e.g., saturation, depolarization block) become significant.

In this cellular formalism, the stochastic DEs (25) to (31) are equivalent to the Fokker-Planck equation,

$$
\begin{aligned}
& \frac{\partial P}{\partial t}=\left[\sum _ { b } \left\{-\sum_{\vec{j}, \vec{k}} \frac{\partial}{\partial V_{b, \vec{j}}} D_{b}^{\vec{j} \vec{k}} V_{b, \vec{k}}+\sum_{\vec{j}} \frac{\partial}{\partial V_{b, \vec{j}}}\left[g\left(V_{b, \vec{j}}\right)\left(V_{b, \vec{j}}-E_{\mathrm{Na}}\right)-I_{b}^{\mathrm{dc}} / C+R_{b, \vec{j}} g_{R, b}\left(V_{b, \vec{j}}-E_{\mathrm{K}}\right)-\sum_{a} \rho_{a} \Phi_{a b, \vec{j}}\right]\right.\right. \\
& \left.+\frac{\Gamma_{b}}{2 \ell^{2}} \sum_{\vec{j}} \frac{\partial^{2}}{\partial V_{b, \vec{j}}^{2}}\right\}+\sum_{b} \sum_{\vec{j}}\left\{\frac{1}{\tau_{b}} \frac{\partial}{\partial R_{b, \vec{j}}}\left[R_{b, \vec{j}}-R_{\infty}\left(V_{b, \vec{j}}\right)\right]-\sum_{\vec{k}} \frac{\partial}{\partial R_{b, \vec{j}}} D_{R}^{\overrightarrow{j k}} V_{b, \vec{k}}+\frac{\Gamma_{R}}{2 \ell^{2}} \frac{\partial^{2}}{\partial R_{b, \vec{j}}^{2}}\right\} \\
& +\sum_{a, b} \sum_{\vec{j}}\left\{-\frac{\partial}{\partial \Phi_{a b, \vec{j}}} Z_{a b, \vec{j}}+\frac{\partial}{\partial Z_{a b, \vec{j}}}\left[\left(\alpha_{a b}+\beta_{a b}\right) Z_{a b, \vec{j}}+\alpha_{a b} \beta_{a b} \Phi_{a b, \vec{j}}-\alpha_{a b} \beta_{a b} N_{a b} \psi_{a b, \vec{j}} \phi_{a b, \vec{j}}\right]+\frac{\Gamma_{Z_{a b}}}{2 \ell^{2}} \frac{\partial^{2}}{\partial Z_{a b, \vec{j}}^{2}}\right\} \\
& +\sum_{a, b} \sum_{\vec{j}}\left\{-\frac{\partial}{\partial \phi_{a b, \vec{j}}} \Pi_{a b, \vec{j}}+\frac{\partial}{\partial \Pi_{a b, \vec{j}}}\left[2 v \Lambda_{a b} \Pi_{a b, \vec{j}}+\left(v \Lambda_{a b}\right)^{2} \phi_{a b, \vec{j}}-\left(v \Lambda_{a b}\right)^{2} S_{a}\left(V_{a, \vec{j}}-V_{a}^{\mathrm{rest}}\right)\right]\right. \\
& \left.\left.-\sum_{\vec{k}} \frac{\partial}{\partial \Pi_{a b, \vec{j}}} D_{\phi_{b}}^{\overrightarrow{j k}} \phi_{a b, \vec{k}}+\frac{\Gamma_{\Pi_{a b}}}{2 \ell^{2}} \frac{\partial^{2}}{\partial \Pi_{a b, \vec{j}}^{2}}\right\}\right] P
\end{aligned}
$$

where $P$ is the probability distribution,

$$
P \equiv P(\chi, t), \quad \chi \in\left\{V_{b, \vec{j}}, R_{b, \vec{j}}, \Phi_{a b, \vec{j}}, Z_{a b, \vec{j}}, \phi_{a b, \vec{j}}, \Pi_{a b, \vec{j}}\right\},
$$

with $\chi$ standing for any one of the 20 state variables [12 system variables $\left(V_{b, \vec{j}}, R_{b, \vec{j}}, \Phi_{a b, \vec{j}}, \phi_{a b, \vec{j}}\right)$ plus eight ancillaries 

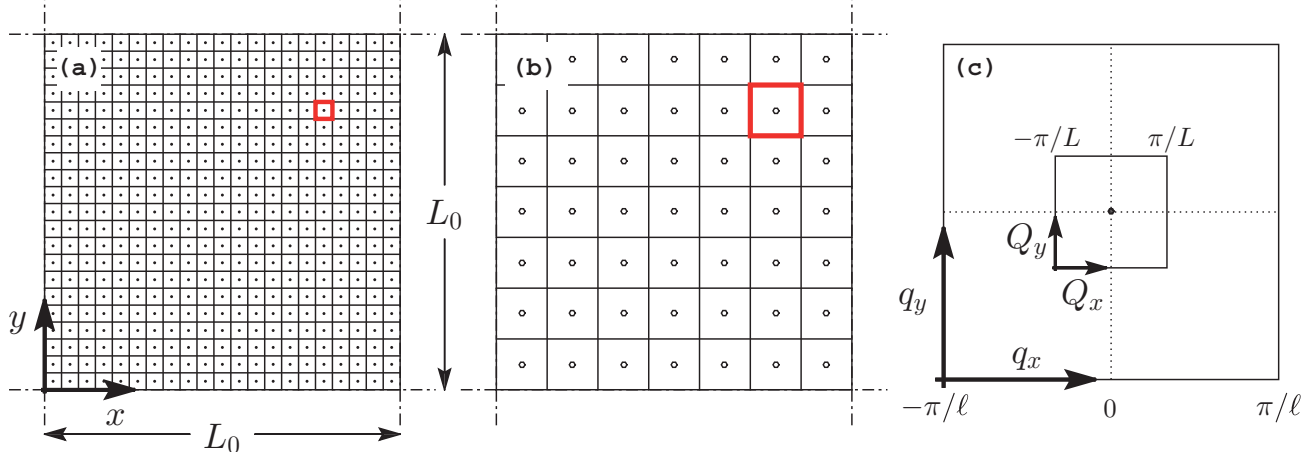

FIG. 4. Spatial and Fourier representations of a gridded cortex. (a) Microcell lattice: A square patch of cortex of dimension $L_{0} \times L_{0}$, assumed periodic, is partitioned into a lattice of $(2 n+1) \times(2 n+1)$ "microcells," each of side length $\ell$ (small red square) such that $L_{0}=(2 n+1) \ell$. (b) Macrocell lattice: The cortical patch is reblocked into $(2 N+1) \times(2 N+1)$ "macrocells," each of side length $L=B \ell$ (larger red square) so that $L_{0}=(2 N+1) L$, with $B=(2 n+1) /(2 N+1) \gg 1$. (For illustrative purposes, we have set $B=3$ in this figure.) (c) Fourier lattice: In Fourier space, these griddings correspond to wave-number domains $\left|q_{x}\right|,\left|q_{y}\right| \leqslant \pi / \ell$ for the finely spaced microgrid, and the much reduced domain $\left|Q_{x}\right|,\left|Q_{y}\right| \leqslant \pi / L$ for the coarse macrogrid.

$\left.\left(Z_{a b, \vec{j}}, \Pi_{a b, \vec{j}}\right)\right]$ whose subscripts $\{a, b\}$ label neural populations $\{e, i\}$. For example, $V_{b, \vec{j}}$ is the voltage for population $b$ of grid cell $\vec{j}$, with $\vec{j}=\left(j_{x}, j_{y}\right)$ being a two-dimensional position index.

$D_{r}^{\vec{j} k}$ is the discretized Laplacian operator given by

$$
\begin{aligned}
D_{r}^{\vec{j} \vec{k}}=\frac{D_{r}}{\ell^{2}}\{ & {\left[\delta_{j_{x}, k_{x}+1}+\delta_{j_{x}, k_{x}-1}-2 \delta_{j_{x}, k_{x}}\right] \delta_{j_{y}, k_{y}} } \\
+ & {\left.\left[\delta_{j_{y}, k_{y}+1}+\delta_{j_{y}, k_{y}-1}-2 \delta_{j_{y}, k_{y}}\right] \delta_{j_{x}, k_{x}}\right\}, }
\end{aligned}
$$

with label $r=e$ and $i$ for $e$-to- $e$ and $i$-to- $i$ diffusion via electrical synapses (gap junctions) and $r=\phi_{b}$ for diffusion via wave-equation activity mediated by chemical synapses.

$D_{R}^{\overrightarrow{j k}}$ is the discretized biharmonic operator,

$$
D_{R}^{\vec{j} k}=\frac{D_{R}}{\ell^{4} \tau_{b}}\left[D_{R, x^{4}}^{\overrightarrow{j k}}+D_{R, x^{2} y^{2}}^{\vec{j} \vec{j}}+D_{R, y^{4}}^{\vec{j} \vec{k}}\right]
$$

where

$$
\begin{aligned}
& D_{R, x^{4}}^{\overrightarrow{j \vec{k}}}=\left[\delta_{j_{x}, k_{x}+2}+\delta_{j_{x}, k_{x}-2}-4\left(\delta_{j_{x}, k_{x}+1}+\delta_{j_{x}, k_{x}-1}\right)\right. \\
& \left.+6 \delta_{j_{x}, k_{x}}\right] \delta_{j_{y}, k_{y}} \text {, } \\
& D_{R, x^{2} y^{2}}^{\vec{j} \vec{k}}=2\left[\delta_{j_{x}, k_{x}+1}+\delta_{j_{x}, k_{x}-1}-2 \delta_{j_{x}, k_{x}}\right] \\
& \times\left[\delta_{j_{y}, k_{y}+1}+\delta_{j_{y}, k_{y}-1}-2 \delta_{j_{y}, k_{y}}\right], \\
& D_{R, y^{4}}^{\overrightarrow{j \vec{k}}}=\left[\delta_{j_{y}, k_{y}+2}+\delta_{j_{y}, k_{y}-2}-4\left(\delta_{j_{x}, k_{y}+1}+\delta_{j_{y}, k_{y}-1}\right)\right. \\
& \left.+6 \delta_{j_{y}, k_{y}}\right] \delta_{j_{x}, k_{x}},
\end{aligned}
$$

with recovery subscript $R$ indexing over both the excitatory and inhibitory neural populations, $R \in\left\{R_{e}, R_{i}\right\}$.

Coarse spatial graining is done rigorously by eliminating the high-wave-number $(\vec{q})$ modes, so the first step is to Fourier transform Eq. (32) to momentum space. Following Steyn-Ross and Gardiner [33] (hereafter abbreviated as SR\&G), we define eigenfunctions and eigenvalues of the discretized Laplacian and biharmonic operators. For the Laplacian, we have

$$
\sum_{\vec{k}} D_{r}^{\vec{j} \vec{k}} f_{\vec{k}}(\vec{q})=-\lambda_{r}(\vec{q}) f_{\vec{j}}(\vec{q})
$$

where the $f_{\vec{k}}$ are the eigenfunctions given by

$$
f_{\vec{k}}(\vec{q})=\frac{1}{(2 n+1)} e^{i \ell \vec{k} \cdot \vec{q}},
$$

with indexing,

$$
k_{x}, k_{y} \in\{-n,-n+1, \ldots, n\} ;
$$

discrete wave-number components,

$$
q_{x}, q_{y} \in \mathscr{R}(n)=\frac{2 \pi}{(2 n+1) \ell}[-n,-n+1, \ldots, n] ;
$$

and $\lambda_{r}$ eigenvalues,

$$
\lambda_{r}(\vec{q})=\frac{4 D_{r}}{\ell^{2}}\left[\sin ^{2}\left(q_{x} \ell / 2\right)+\sin ^{2}\left(q_{y} \ell / 2\right)\right] .
$$

For the biharmonic operator we have

$$
\sum_{\vec{k}} D_{R}^{\overrightarrow{j k}} f_{\vec{k}}(\vec{q})=\lambda_{R}(\vec{q}) f_{\vec{j}}(\vec{q}),
$$

where the $f_{\vec{k}}$ eigenfunctions are defined in Eq. (34b); the corresponding eigenvalues are given by

$$
\lambda_{R}(\vec{q})=\frac{16 D_{R}}{\ell^{4} \tau_{b}}\left[\sin ^{2}\left(q_{x} \ell / 2\right)+\sin ^{2}\left(q_{y} \ell / 2\right)\right]^{2} .
$$

The eigenfunctions obey the orthogonality relations

$$
\begin{gathered}
\sum_{\vec{j}} f_{\vec{j}}^{*}(\vec{q}) f_{\vec{j}}\left(\vec{q}^{\prime}\right)=\delta_{\vec{q}, \vec{q}^{\prime}}, \\
\sum_{\vec{q}} f_{\vec{j}}^{*}(\vec{q}) f_{\vec{k}}(\vec{q})=\delta_{\vec{j}, \vec{k}},
\end{gathered}
$$

with symbol $\left(^{*}\right)$ denoting complex conjugate. We then transform to Fourier $(\vec{q})$ space via the expansions:

$$
\chi_{\vec{j}}=\sum_{\vec{q}} f_{\vec{j}}(\vec{q}) \chi(\vec{q}), \quad \chi(\vec{q})=\sum_{\vec{j}} f_{\vec{j}}^{*}(\vec{q}) \chi_{\vec{j}} .
$$

The aim of this paper is to eliminate the high spatial frequency modes; therefore, we separate momentum scales 
explicitly into low-frequency (long-wavelength) $\vec{Q}$ modes and high-frequency (small-wavelength) $\vec{q}$-mode components,

$$
\sum_{\vec{q}} \chi(\vec{q}) \longrightarrow \sum_{\vec{Q} \in \mathscr{R}(N)} \chi(\vec{Q})+\sum_{\vec{q} \notin \mathscr{R}(N)} \chi(\vec{q}),
$$

where

$$
Q_{i} \in \mathscr{R}(N)=\frac{2 \pi}{(2 n+1) \ell}[-N,-N+1, \ldots, N] .
$$

As illustrated in Figs. 4(b) and 4(c), the blocking procedure regrids the $L_{0} \times L_{0}$ cortical patch into $(2 N+1)^{2}$ macrocolumn cells ("macrocells"), each of area $L^{2}$, where $L=B \ell \sim 1 \mathrm{~mm}$; $B$ is the blocking ratio,

$$
B=\frac{2 n+1}{2 N+1} \gg 1 .
$$

Using Eqs. (34)-(35), Eq. (32) becomes

$$
\frac{\partial}{\partial t} P(\chi, t)=\left(L_{1}+L_{2}+L_{3}\right) P(\chi, t),
$$

where the three $L_{j}$ terms have been chosen to allow implementation of the elimination procedure, as detailed in the sections that follow. In particular,

$$
\begin{aligned}
L_{1}= & \sum_{b}\left\{\sum_{\vec{q}} \frac{\partial}{\partial V_{b}(\vec{q})}\left[\lambda_{b}(\vec{q}) V_{b}(\vec{q})+a_{0, b} V_{b}(\vec{q})-g_{R, b} E_{\mathrm{K}} R_{b}(\vec{q})\right]+\sum_{\vec{q}} \frac{\partial}{\partial R_{b}(\vec{q})}\left[-\lambda_{R}(\vec{q}) V_{b}(\vec{q})-\frac{b_{1, b}}{\tau_{b}} V_{b}(\vec{q})\right]\right. \\
& \left.+\frac{\Gamma_{b}}{2 \ell^{2}} \sum_{\vec{q}} \frac{\partial^{2}}{\partial V_{b}(\vec{q}) V_{b}(-\vec{q})}\right\}+\sum_{a, b}\left\{-\sum_{\vec{q}} \frac{\partial}{\partial \Phi_{a b}(\vec{q})} Z_{a b}(\vec{q})+\sum_{\vec{q}} \frac{\partial}{\partial Z_{a b}(\vec{q})}\left[\left(\alpha_{a b}+\beta_{a b}\right) Z_{a b}(\vec{q})+\alpha_{a b} \beta_{a b} \Phi_{a b}(\vec{q})\right]\right. \\
& \left.+\frac{\Gamma_{Z}}{2 \ell^{2}} \sum_{\vec{q}} \frac{\partial^{2}}{\partial Z_{a b}(\vec{q}) Z_{a b}(-\vec{q})}\right\}+\sum_{a, b}\left\{-\sum_{\vec{q}} \frac{\partial}{\partial \phi_{a b}(\vec{q})} \Pi_{a b}(\vec{q})+\sum_{\vec{q}} \frac{\partial}{\partial \Pi_{a b}(\vec{q})}\left[2 v \Lambda_{a b} \Pi_{a b}(\vec{q})\right.\right. \\
& \left.\left.+\left(\left(v \Lambda_{a b}\right)^{2}+\lambda_{\phi}(\vec{q})\right) \phi_{a b}(\vec{q})\right]+\frac{\Gamma_{\Pi}}{2 \ell^{2}} \sum_{\vec{q}} \frac{\partial^{2}}{\partial \Pi_{a b}(\vec{q}) \Pi_{a b}(-\vec{q})}\right\}
\end{aligned}
$$

has been constructed to define the stationary distribution function as the sum of a set of 2D Ornstein-Uhlenbeck (or Kramer) solutions; this construction is the cornerstone of the projection operator that eliminates the high- $q$ spatial modes,

$$
\begin{aligned}
& L_{2}=\sum_{b}\left\{\sum _ { [ \vec { Q } , \vec { q } ] } \frac { \partial } { \partial V _ { b } ( \vec { q } ) } \left(-\sum_{a} \rho_{a} \Phi_{a b}(\vec{q})+\frac{a_{3, b}}{(2 n+1)}\left[V_{b}\left(\vec{q}_{1}\right) V_{b}\left(\vec{q}_{2}\right) \delta_{\vec{q}, \vec{q}_{1}+\vec{q}_{2}}+2 V_{b}\left(\vec{q}_{1}\right) V_{b}\left(\vec{Q}_{2}\right) \delta_{\vec{q}, \vec{q}_{1}+\vec{Q}_{2}}\right.\right.\right. \\
& \left.+V_{b}\left(\vec{Q}_{1}\right) V_{b}\left(\vec{Q}_{2}\right) \delta_{\vec{q}, \vec{Q}_{1}+\vec{Q}_{2}}\right]-a_{1, b} E_{\mathrm{Na}} V_{b}(\vec{q})+\frac{a_{2, b}}{(2 n+1)^{2}}\left[V_{b}\left(\vec{q}_{1}\right) V_{b}\left(\vec{q}_{2}\right) V_{b}\left(\vec{q}_{3}\right) \delta_{\vec{q}, \vec{q}_{1}+\vec{q}_{2}+\vec{q}_{3}}\right.
\end{aligned}
$$

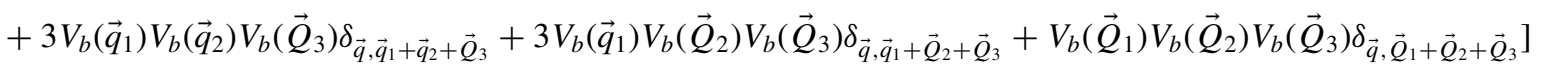

$$
\begin{aligned}
& +\frac{g_{R, b}}{(2 n+1)}\left[R_{b}\left(\vec{q}_{1}\right) V_{b}\left(\vec{q}_{2}\right) \delta_{\vec{q}, \vec{q}_{1}+\vec{q}_{2}}+R_{b}\left(\vec{Q}_{1}\right) V_{b}\left(\vec{q}_{2}\right) \delta_{\vec{q}, \vec{Q}_{1}+\vec{q}_{2}}+R_{b}\left(\vec{q}_{1}\right) V_{b}\left(\vec{Q}_{2}\right) \delta_{\vec{q}, \vec{q}_{1}+\vec{Q}_{2}}+R_{b}\left(\vec{Q}_{1}\right) V_{b}\left(\vec{Q}_{2}\right) \delta_{\left.\left.\vec{q}, \vec{Q}_{1}+\vec{Q}_{2}\right]\right)}\right. \\
& +\sum_{[\vec{Q}, \vec{q}]} \frac{\partial}{\partial R_{b}(\vec{q})}\left(\frac{1}{\tau_{b}} R_{b}(\vec{q})-\frac{b_{2, b}}{\tau_{b}(2 n+1)}\left[V_{b}\left(\vec{q}_{1}\right) V_{b}\left(\vec{q}_{2}\right) \delta_{\vec{q}, \vec{q}_{1}+\vec{q}_{2}}+2 V_{b}\left(\vec{q}_{1}\right) V_{b}\left(\vec{Q}_{2}\right) \delta_{\vec{q}_{,} \vec{q}_{1}+\vec{Q}_{2}}+V_{b}\left(\vec{Q}_{1}\right) V_{b}\left(\vec{Q}_{2}\right) \delta_{\left.\left.\vec{q}, \vec{Q}_{1}+\vec{Q}_{2}\right]\right)}\right.\right. \\
& \left.+\frac{\Gamma_{R}}{2 \ell^{2}} \sum_{\vec{q}} \frac{\partial^{2}}{\partial R_{b}(\vec{q}) \partial R_{b}(-\vec{q})}\right\}+\sum_{a, b}\left\{-\sum_{\vec{q}} \frac{\partial}{\partial \Pi_{a b}(\vec{q})} S_{a}\left(v \Lambda_{a b}\right)^{2} V_{a}(\vec{q})+\sum_{\vec{q}} \frac{\partial}{\partial Z_{a b}(\vec{q})}\left(-b_{a b}\left(V_{a}^{\mathrm{rev}}-\bar{V}_{b}\right) \phi_{a b}(\vec{q})\right)\right\},
\end{aligned}
$$

with constants $a_{3, b}$ and $b_{a b}$ defined by

$$
\begin{gathered}
a_{3, b}=a_{1, b}-a_{2, b} E_{\mathrm{Na}}, \\
b_{a b}=\frac{\alpha_{a b} \beta_{a b} N_{a b}}{V_{a}^{\text {rev }}-V_{b}^{\text {rest }} .}
\end{gathered}
$$

We note that operator $L_{2}$ has been constructed so that it only contains terms of the form $\partial / \partial \chi(\vec{q})$; this form is also essential for the elimination of the high-frequency modes (see Sec. IIE).

$L_{3}$ is expressed as the sum of a mixed-mode operator $\mathscr{L}_{3}(\vec{q}, \vec{Q})$ plus a slow-mode operator $L_{3}(\vec{Q})$,

$$
L_{3}=\mathscr{L}_{3}(\vec{q}, \vec{Q})+L_{3}(\vec{Q})
$$


where the slow-mode operator is defined,

$$
\begin{aligned}
& L_{3}(\vec{Q})=\sum_{b}\left\{\sum_{\vec{Q}} \frac{\partial}{\partial V_{b}(\vec{Q})} \lambda_{b}(\vec{Q}) V(\vec{Q})+\sum_{\vec{Q}} \frac{\partial}{\partial V_{b}(\vec{Q})}\left(-a_{0, b} E_{\mathrm{Na}}(2 n+1) \delta_{Q, 0}+\left(a_{0, b}-a_{1, b} E_{\mathrm{Na}}\right) V_{b}(\vec{Q})-(2 n+1) \delta_{Q, 0} I_{b}^{\mathrm{dc}} / C\right.\right. \\
& -g_{R, b} E_{\mathrm{K}} R_{b}(\vec{Q})-\sum_{a} \rho_{a} \Phi_{a b}(\vec{Q})+\frac{a_{3, b}}{(2 n+1)} V_{b}\left(\vec{Q}_{1}\right) V_{b}\left(\vec{Q}_{2}\right) \delta_{\vec{Q}, \vec{Q}_{1}+\vec{Q}_{2}}+\frac{a_{2, b}}{(2 n+1)^{2}} V_{b}\left(\vec{Q}_{1}\right) V_{b}\left(\vec{Q}_{2}\right) V_{b}\left(\vec{Q}_{3}\right) \delta_{\vec{Q}, \vec{Q}_{1}+\vec{Q}_{2}+\vec{Q}_{3}} \\
& \left.+\frac{g_{R, b}}{(2 n+1)} R_{b}\left(\vec{Q}_{1}\right) V_{b}\left(\vec{Q}_{2}\right) \delta_{\vec{Q}, \vec{Q}_{1}+\vec{Q}_{2}}\right)+\frac{\Gamma_{b}}{2 \ell^{2}} \sum_{\vec{Q}} \frac{\partial^{2}}{\partial V_{b}(\vec{Q}) V_{b}(-\vec{Q})}+\sum_{\vec{Q}} \frac{\partial}{\partial R_{b}(\vec{Q})}\left(\frac{1}{\tau_{b}} R_{b}(\vec{Q})-\frac{b_{0, b}}{\tau_{b}}(2 n+1) \delta_{\vec{Q}, 0}\right. \\
& \left.\left.-\frac{b_{1, b}}{\tau_{b}} V_{b}(\vec{Q})-\lambda_{R}(\vec{Q}) V_{b}(\vec{Q})-\frac{b_{2, b}}{\tau_{b}(2 n+1)} V_{b}\left(\vec{Q}_{1}\right) V_{b}\left(\vec{Q}_{2}\right) \delta_{\vec{Q}, \vec{Q}_{1}+\vec{Q}_{2}}\right)+\frac{\Gamma_{R}}{2 \ell^{2}} \sum_{\vec{Q}} \frac{\partial^{2}}{\partial R_{b}(\vec{Q}) R_{b}(-\vec{Q})}\right\} \\
& +\sum_{a, b}\left\{-\sum_{\vec{Q}} \frac{\partial}{\partial \Phi_{a b}(\vec{Q})} Z_{a b}(\vec{Q})+\sum_{\vec{Q}} \frac{\partial}{\partial Z_{a b}(\vec{Q})}\left(\left(\alpha_{a b}+\beta_{a b}\right) Z_{a b}(\vec{Q})+\alpha_{a b} \beta_{a b} \Phi_{a b}(\vec{Q})-b_{a b}\left(V_{a}^{\mathrm{rev}}-\bar{V}_{b}\right) \phi_{a b}(\vec{Q})\right)\right. \\
& +\frac{\Gamma_{Z}}{2 \ell^{2}} \sum_{\vec{Q}} \frac{\partial^{2}}{\partial Z_{a b}(\vec{Q}) Z_{a b}(-\vec{Q})}-\sum_{\vec{Q}} \frac{\partial}{\partial \phi_{a b}(\vec{Q})} \Pi_{a b}(\vec{Q})+\sum_{\vec{Q}} \frac{\partial}{\partial \Pi_{a b}(\vec{Q})}\left(2 v \Lambda_{a b} \Pi_{a b}(\vec{Q})+\left(v \Lambda_{a b}\right)^{2} \phi_{a b}(\vec{Q})\right. \\
& \left.\left.-S_{a} \cdot\left(v \Lambda_{a b}\right)^{2}\left(V_{a}(\vec{Q})-V_{a}^{\mathrm{rest}}(2 n+1) \delta_{\vec{Q}, 0}\right)\right)+\sum_{\vec{Q}} \frac{\partial}{\partial \Pi_{a b}(\vec{Q})} \lambda_{\phi}(\vec{Q}) \phi_{a b}(\vec{Q})+\frac{\Gamma_{\Pi}}{2 \ell^{2}} \sum_{\vec{Q}} \frac{\partial^{2}}{\partial \Pi_{a b}(\vec{Q}) \Pi_{a b}(-\vec{Q})}\right\} .
\end{aligned}
$$

Meanwhile, the mixed-mode component of $L_{3}$ is given by

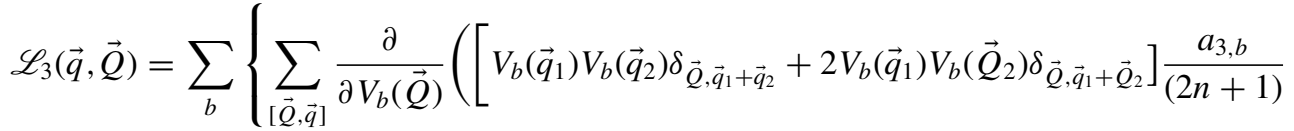

$$
\begin{aligned}
& +\frac{a_{2, b}}{(2 n+1)^{2}}\left[V_{b}\left(\vec{q}_{1}\right) V_{b}\left(\vec{q}_{2}\right) V_{b}\left(\vec{q}_{3}\right) \delta_{\vec{Q}, \vec{q}_{1}+\vec{q}_{2}+\vec{q}_{3}}+3 V_{b}\left(\vec{q}_{1}\right) V_{b}\left(\vec{q}_{2}\right) V_{b}\left(\vec{Q}_{3}\right) \delta_{\vec{Q}_{,}, \vec{q}_{1}+\vec{q}_{2}+\vec{Q}_{3}}\right. \\
& \left.+3 V_{b}\left(\vec{q}_{1}\right) V_{b}\left(\vec{Q}_{2}\right) V_{b}\left(\vec{Q}_{3}\right) \delta_{\vec{Q}_{,} \vec{q}_{1}+\vec{Q}_{2}+\vec{Q}_{3}}\right]+\frac{g_{R, b}}{(2 n+1)}\left[R_{b}\left(\vec{q}_{1}\right) V_{b}\left(\vec{q}_{2}\right) \delta_{\vec{Q}, \vec{q}_{1}+\vec{q}_{2}}+R_{b}\left(\vec{Q}_{1}\right) V_{b}\left(\vec{q}_{2}\right) \delta_{\vec{Q}, \vec{Q}_{1}+\vec{q}_{2}}\right. \\
& \left.\left.\left.+R_{b}\left(\vec{q}_{1}\right) V_{b}\left(\vec{Q}_{2}\right) \delta_{\vec{Q}, \vec{q}_{1}+\vec{Q}_{2}}\right]\right)-\frac{b_{2, b}}{\tau_{b}(2 n+1)} \sum_{\vec{Q}} \frac{\partial}{\partial R_{b}(\vec{Q})}\left(V_{b}\left(\vec{q}_{1}\right) V_{b}\left(\vec{q}_{2}\right) \delta_{\vec{Q}, \vec{q}_{1}+\vec{q}_{2}}+2 V_{b}\left(\vec{q}_{1}\right) V_{b}\left(\vec{Q}_{2}\right) \delta_{\vec{Q}, \vec{q}_{1}+\vec{Q}_{2}}\right)\right\} .
\end{aligned}
$$

We note that $L_{3}(\vec{Q})$ is the Fourier representation of the original FPE on the coarser spatial scale, and $\mathscr{L}_{3}(\vec{Q})$ contains all remaining terms.

\section{E. Adiabatic elimination of high $-\vec{q}$ modes}

We follow the method of projection-operator elimination described by Gardiner [32] and SR\&G [33]. The method proceeds by first constructing a projection operator via the stationary distribution $P_{s}$ of the variables to be eliminated. The procedure is summarized here; refer to [33] for details.

We define a projection operator $\mathscr{P}$,

$$
\mathscr{P} u(\vec{q}, \vec{Q}) \equiv P_{s} \int u(\vec{q}, \vec{Q}) d \chi(\vec{q}),
$$

where $u(\vec{q}, \vec{Q})$ is an arbitrary function and $P_{s}$ is the solution of

$$
L_{1} P_{s}=0,
$$

so that,

$$
L_{1} \mathscr{P}=\mathscr{P} L_{1}=0,
$$

and also from Eq. (40) we have that

$$
\mathscr{P} L_{2} \mathscr{P}=0, \quad \mathscr{P} L_{2}=0,
$$

which follows since $L_{2}$ will produce only boundary terms, which, by definition, will vanish (see [33]).

We define

$$
\begin{aligned}
v(t) & \equiv \mathscr{P} P(\chi, t), \quad \text { and } \\
w(t) & \equiv(1-\mathscr{P}) P(\chi, t) ;
\end{aligned}
$$

then, applying the projection operator $\mathscr{P}$ to the full FokkerPlanck equation (38), we find

$$
\mathscr{P} \frac{\partial}{\partial t} P(\chi, t)=\mathscr{P} L_{3} P(\chi, t),
$$

so that

$$
\frac{\partial}{\partial t} \mathscr{P} P(\chi, t)=\mathscr{P} L_{3}[w(t)+v(t)]
$$


which is equivalent to

$$
\frac{\partial}{\partial t} v(t)=\mathscr{P} L_{3}[w(t)+v(t)]
$$

Similarly,

$$
\begin{aligned}
(1 & -\mathscr{P}) \frac{\partial}{\partial t} P(\chi, t) \\
& =(1-\mathscr{P})\left(L_{1} P+L_{2} P+L_{3} P\right) \\
& =L_{1}(w+v)+L_{2}(w+v)+(1-\mathscr{P}) L_{3}(w+v) \\
& =L_{1} w+L_{2} w+(1-\mathscr{P}) L_{3} w+(1-\mathscr{P}) L_{3} v+L_{2} v
\end{aligned}
$$

(since $L_{1} v=L_{1} \mathscr{P} P=0$ ), implying that

$$
\frac{\partial}{\partial t} w(t)=\left[L_{1}+L_{2}+(1-\mathscr{P}) L_{3}\right] w+(1-\mathscr{P}) L_{3} v+L_{2} v .
$$

Defining Laplace-transformed variables

$$
v(s)=\widehat{\mathrm{L}}[v(t)] \equiv \int_{0}^{\infty} e^{-s t} v(t) d t
$$

and similarly $w(s)=\widehat{\mathrm{L}}[w(t)]$, the Laplace transforms of Eqs (49a) and (49b) give

$$
\begin{aligned}
s v(s)-v(0)= & \mathscr{P} L_{3} w(s)+\mathscr{P} L_{3} v(s) \\
s w(s)= & {\left[L_{1}+L_{2}+(1-\mathscr{P}) L_{3}\right] w(s) } \\
& +\left[L_{2}+(1-\mathscr{P}) L_{3}\right] v(s),
\end{aligned}
$$

where $v(0)$ denotes $v(t)$ at $t=0$, and $w(0)=0$. Solving Eq. (50b) for $w(s)$,

$$
\begin{aligned}
w(s)= & {\left[s-L_{1}-L_{2}-(1-\mathscr{P}) L_{3}\right]^{-1} } \\
& \times\left[L_{2}+(1-\mathscr{P}) L_{3}\right] v(s),
\end{aligned}
$$

and substituting for $w(s)$ in Eq. (50a),

$$
\begin{aligned}
s v(s)-v(0)= & \mathscr{P} L_{3}\left[s-L_{1}-L_{2}-(1-\mathscr{P}) L_{3}\right]^{-1} \\
& \times\left[L_{2}+(1-\mathscr{P}) L_{3}\right] v(s)+\mathscr{P} L_{3} v(s) .
\end{aligned}
$$

Following SR\&G [33], we assume that the elementary cell length $\ell$ is small, that the blocking ratio $B=(2 n+$ $1) /(2 N+1)$ is large, and that there exists a nonzero diffusive gap-junction coupling between nearest neighbors. (See Appendix $\mathrm{F}$ for an estimate of the minimum level of gap-junction connectivity required for validity of the blocking algorithm assumptions.)

Under these conditions, $L_{1}$ dominates the denominator of Eq. (50d), and it simplifies to

$s v(s)-v(0)=-\mathscr{P} L_{3} L_{1}^{-1}\left[L_{2}+(1-\mathscr{P}) L_{3}\right] v(s)+\mathscr{P} L_{3} v(s)$,

whose inverse Laplace transform gives the Fokker-Planck equation on the coarse-grained grid,

$$
\frac{\partial}{\partial t} v=-\mathscr{P} L_{3} L_{1}^{-1}\left[L_{2}+(1-\mathscr{P}) L_{3}\right] v(t)+\mathscr{P} L_{3} v(t) .
$$

\section{F. Rescaled Fokker-Planck equation}

Equation (51) is a regridded Fokker-Planck equation describing a system in which short-wavelength (high-spatial frequency) $q$ modes have been eliminated in favor of longwavelength $Q$ modes. It describes the time evolution of $v(t)$,

$$
\begin{aligned}
v(t) & =\mathscr{P} P(\chi, t) \\
& =P_{s} \int P(\chi, t) d \chi(\vec{q})=P_{s} \widehat{P}(\chi(\vec{Q}, t)),
\end{aligned}
$$

where $\widehat{P}(\chi(\vec{Q}, t))$ is the $\vec{Q}$-space probability distribution for the spatially averaged system. We invert $v(t)$ from $\vec{Q}$ space back to $\vec{r}$ space by evaluating the RHS of Eq. (51) in terms of expectation values in the "vacuum" state of the cortex. This involves a painstaking and rather technical process that is detailed in the Appendixes. We present a brief overview of the method here.

We construct the projection operator $\mathscr{P}$ of Eq. (46) from $P_{s}$, the stationary solution of the $L_{1}$ operator of Eq. (39),

$$
L_{1} P_{s}=0 \text {. }
$$

To do this, we write $L_{1}$ as

$$
L_{1}=L_{1}(V, R)+L_{1}(Z, \Phi)+L_{1}(\Pi, \phi),
$$

so that separation of variables allows us to express the stationary solution as

$$
P_{s}=P_{s}(V, R) P_{s}(Z, \Phi) P_{s}(\Pi, \phi) .
$$

Each term on the RHS of (53) has the form of Kramer's equation. This particular two-variable Ornstein-Uhlenbeck form has been extensively studied $[29,47]$; it is known that each of the $P_{s}(\cdot)$ components on the RHS of (54) can be expressed as a sum of Hermite polynomials; thus, each of the $L_{1}(\cdot)$ terms of (53) represents a process formally equivalent to the harmonic oscillator. This means that we can adopt the elegant boson-operator algebra used to solve the quantum mechanical harmonic oscillator, with all variables being expressible in terms of boson creation and annihilation operators $\left(A^{\dagger}, A\right)$; see Appendix B for details.

Because it can be shown that

$$
A P_{s}=0 \text {, }
$$

it immediately follows that $P_{S}$ is precisely equivalent to the vacuum, or ground state $|0\rangle$, defined as

$$
\begin{aligned}
P_{s} & =|0\rangle \\
& =|0\rangle_{V, R}|0\rangle_{Z, \Phi}|0\rangle_{\Pi, \phi} .
\end{aligned}
$$

Thus, $|0\rangle$ can be interpreted as the ground state in a Fock space equivalent to our cortical system. The terms on the RHS of Eq. (51) can then be expressed as vacuum-state expectation values of operators.

We thus arrive at the coarse-grained Fokker-Planck equation,

$$
\frac{\partial \widehat{P}}{\partial t}=\left.\frac{\partial \widehat{P}}{\partial t}\right|_{(1)}-\left.\frac{\partial \widehat{P}}{\partial t}\right|_{(2 \text { :drift })}-\left.\frac{\partial \widehat{P}}{\partial t}\right|_{(2 \text { :diffusion })},
$$

where the first term on the RHS is the spatially rescaled version of Eq. (32), and the drift and diffusion terms represent corrections due to nonlinear interactions between spatial 
modes. The first term on the right of Eq. (57) is given by

$$
\begin{aligned}
\left.\frac{\partial \widehat{P}}{\partial t}\right|_{(1)}= & {\left[\left\{-\sum_{\vec{J}, \vec{K}} \frac{\partial}{\partial \widetilde{V}_{b, \vec{J}}} D_{b}^{\vec{J} \vec{K}} \widetilde{V}_{b, \vec{K}}+\sum_{\vec{J}} \frac{\partial}{\partial \widetilde{V}_{b, \vec{J}}}\left[g\left(\widetilde{V}_{b, \vec{J}}\right)\left(\widetilde{V}_{b, \vec{J}}-E_{\mathrm{Na}}\right)-I_{b}^{\mathrm{dc}} / C+\widetilde{R}_{b, \vec{J}} g_{R, b}\left(\widetilde{V}_{b, \vec{J}}-E_{\mathrm{K}}\right)-\sum_{a} \rho_{a} \widetilde{\Phi}_{a b, \vec{J}}\right]\right.\right.} \\
& \left.+\frac{\Gamma_{b}}{2(B \ell)^{2}} \sum_{\vec{J}} \frac{\partial^{2}}{\partial \widetilde{V}_{b, \vec{j}}^{2}}\right\}+\sum_{b} \sum_{\vec{J}}\left\{\frac{1}{\tau_{b}} \frac{\partial}{\partial \widetilde{R}_{b, \vec{J}}}\left[\widetilde{R}_{b, \vec{J}}-R_{\infty}\left(\widetilde{V}_{b, \vec{J}}\right)\right]-\sum_{\vec{K}} \frac{\partial}{\partial \widetilde{R}_{b, \vec{J}}} D_{R}^{\vec{J} \vec{K}} \widetilde{V}_{b, \vec{K}}+\frac{\Gamma_{R}}{2(B \ell)^{2}} \frac{\partial^{2}}{\partial \widetilde{R}_{b, \vec{J}}^{2}}\right\} \\
& +\sum_{a, b} \sum_{\vec{J}}\left\{-\frac{\partial}{\partial \widetilde{\Phi}_{a b, \vec{J}}} \widetilde{Z}_{a b, \vec{J}}+\frac{\partial}{\partial \widetilde{Z}_{a b, \vec{J}}}\left[\left(\alpha_{a b}+\beta_{a b}\right) \widetilde{Z}_{a b, \vec{J}}+\alpha_{a b} \beta_{a b} \widetilde{\Phi}_{a b, \vec{J}}-\alpha_{a b} \beta_{a b} N_{a b} \widetilde{\psi}_{a b, \vec{J}} \widetilde{\phi}_{a b, \vec{J}}\right]+\frac{\Gamma_{Z_{a b}}}{2(B \ell)^{2}} \frac{\partial^{2}}{\partial \widetilde{Z}_{a b, \vec{J}}^{2}}\right\} \\
& +\sum_{a, b} \sum_{\vec{J}}\left\{-\frac{\partial}{\partial \widetilde{\phi}_{a b, \vec{J}}} \widetilde{\Pi}_{a b, \vec{J}}+\frac{\partial}{\partial \widetilde{\Pi}_{a b, \vec{J}}}\left[2 v \Lambda_{a b} \widetilde{\Pi}_{a b, \vec{J}}+\left(v \Lambda_{a b}\right)^{2} \widetilde{\phi}_{a b, \vec{J}}-\left(v \Lambda_{a b}\right)^{2} S_{a} \cdot\left(\widetilde{V}_{a, \vec{J}}-V_{a}^{\mathrm{rest}}\right)\right]\right. \\
& \left.\left.-\sum_{\vec{K}} \frac{\partial}{\partial \widetilde{\Pi}_{a b, \vec{J}}} D_{\phi_{b} \vec{K} \vec{\phi}} \widetilde{\phi}_{a b, \vec{K}}+\frac{\Gamma_{\Pi_{a b}}}{2(B \ell)^{2}} \frac{\partial^{2}}{\partial \widetilde{\Pi}_{a b, \vec{J}}^{2}}\right\}\right] \widehat{P}_{(58)}
\end{aligned}
$$

where the tilde variables denote the spatially rescaled system variables of Eq. (32), i.e.,

$$
[V, R, Z, \Phi, \Pi, \phi] \longrightarrow[\widetilde{V}, \widetilde{R}, \widetilde{Z}, \widetilde{\Phi}, \widetilde{\Pi}, \widetilde{\phi}]
$$

corresponding to an upscaling of the area of the unit cell from $\ell^{2}$ in Eq. (32) to $(B \ell)^{2}$ here; recall that $B$ is the side-length blocking ratio defined in Eq. (37). We note that the constant-intensity noise contributions from Eq. (32) become downscaled in Eq. (58) by the area ratio; thus,

$$
\Gamma_{[b, R, Z, \Pi]} / \ell^{2} \longrightarrow \Gamma_{[b, R, Z, \Pi]} /(B \ell)^{2} .
$$

The nonlinear drift corrections of $\partial \widehat{P} / \partial t$ in Eq. (57) are given by,

$$
\begin{aligned}
& \left.\frac{\partial \widehat{P}}{\partial t}\right|_{(2: \text { drift })} \\
& =\sum_{b, \vec{J}}\left\{\frac { \partial } { \partial \widetilde { V } _ { b , \vec { J } } } \left[d_{0}+d_{1} \widetilde{V}_{b, \vec{J}}+d_{2} \widetilde{V}_{b, \vec{J}}^{2}+d_{3} \widetilde{V}_{b, \vec{J}}^{3}\right.\right. \\
& \left.\quad+d_{4} \widetilde{V}_{b, \vec{J}}^{4}+d_{5} \widetilde{V}_{b, \vec{J}} \widetilde{R}_{b, \vec{J}}+d_{6} \widetilde{V}_{b, \vec{J}}^{2} \widetilde{R}_{b, \vec{J}}+d_{7} \widetilde{R}_{b, \vec{J}}\right] \\
& \quad+\frac{\partial}{\partial \widetilde{R}_{b, \vec{J}}}\left[d_{8}+d_{9} \widetilde{R}_{b, \vec{J}}+d_{10} \widetilde{V}_{b, \vec{J}}\right. \\
& \left.\left.\quad+d_{11} \widetilde{V}_{b, \vec{J}}^{2}+d_{12} \widetilde{V}_{b, \vec{J}}^{3}\right]\right\} \widehat{P} \\
& \equiv \sum_{b, \vec{J}}\left\{\frac{\partial}{\partial \widetilde{V}_{b, \vec{J}}} G_{1}+\frac{\partial}{\partial \widetilde{R}_{b, \vec{J}}} G_{2}\right\} \widehat{P},
\end{aligned}
$$

where the $G_{1}, G_{2}$ encapsulate their respective squarebracketed antecedents. The $d_{0}, \ldots, d_{12}$ drift coefficients [along with their SI units] are listed below,

$$
\begin{aligned}
d_{0}= & \frac{\Gamma_{b}}{2(2 \pi)^{2}}\left[-a_{3, b} \bar{c}_{0}+a_{1, b} a_{3, b} \bar{c}_{1} E_{\mathrm{Na}}\right. \\
& -a_{2, b} a_{3, b} \Gamma_{b}\left(\frac{\bar{c}_{2}}{[(2 n+1) \ell]^{2}}+\frac{\bar{c}_{3}}{2(2 \pi)^{2}}\right)
\end{aligned}
$$

$$
\begin{aligned}
& +\frac{a_{2, b} \Gamma_{b}}{2(2 \pi)^{2}}\left(-\sqrt{\frac{g_{R, b}}{\left|E_{\mathrm{K}}\right|}} \bar{c}_{6 a}+\frac{b_{2, b} \bar{c}_{6 b} \sqrt{g_{R, b}\left|E_{\mathrm{K}}\right|}}{\tau_{b}}\right) \\
& -\frac{a_{3, b} \bar{c}_{7}}{\tau_{b}}+\frac{a_{3, b} \bar{c}_{8} g_{R, b}\left|E_{\mathrm{K}}\right| \Gamma_{R}}{\Gamma_{b}}-\sqrt{\frac{g_{R, b}}{\left|E_{\mathrm{K}}\right|}} \frac{\bar{c}_{9}}{\tau_{b}} \\
& \left.+\frac{\sqrt{g_{R, b}^{3}\left|E_{\mathrm{K}}\right|} \bar{c}_{10} \Gamma_{R}}{\Gamma_{b}}-\frac{3 a_{2, b} a_{3, b} \bar{c}_{11} \Gamma_{b}}{2(B \ell)^{2}}\right]
\end{aligned}
$$

$$
d_{1}=\frac{\Gamma_{b}}{2(2 \pi)^{2}}\left[-3 a_{2, b} \bar{c}_{0}-\left(2 a_{3, b}^{2}-3 a_{1, b} a_{2, b} E_{\mathrm{Na}}\right) \bar{c}_{1}\right.
$$$$
-3 a_{2, b}^{2} \Gamma_{b}\left(\frac{\bar{c}_{2}}{[(2 n+1) \ell]^{2}}+\frac{\bar{c}_{3}}{2(2 \pi)^{2}}\right)
$$$$
-\frac{3 a_{2, b} \bar{c}_{7}}{\tau_{b}}+\frac{3 a_{2, b} \bar{c}_{8} g_{R, b}\left|E_{\mathrm{K}}\right| \Gamma_{R}}{\Gamma_{b}}
$$$$
\left.-\frac{9 a_{2, b}^{2} \bar{c}_{11} \Gamma_{b}}{2(B \ell)^{2}}\right]+\frac{S_{1}^{2} \Gamma_{b}}{2(B \ell)^{2}\left|E_{\mathrm{K}}\right|^{2}}
$$

$$
d_{2}=\frac{3 a_{2, b} \Gamma_{b}}{2(2 \pi)^{2}}\left[-3 a_{3, b} \bar{c}_{1}\right.
$$$$
\left.+\frac{(B \ell)^{2}}{(2 \pi)^{2}\left|E_{\mathrm{K}}\right|}\left(-\bar{c}_{15} S_{2}+\frac{\bar{c}_{16} S_{1}(B \ell)^{2}}{2 \pi}\right)\right]
$$

$$
d_{3}=\frac{a_{3, b} S_{1}^{2}}{\left|E_{\mathrm{K}}\right|}-\frac{9 a_{2, b}^{2} \bar{c}_{1} \Gamma_{b}}{2(2 \pi)^{2}}
$$

$$
+\left[2 a_{3, b} \bar{c}_{4}-\frac{\bar{c}_{5}}{\left|E_{\mathrm{K}}\right|}\right] \frac{b_{2, b} S_{1}(B \ell)^{4}}{(2 \pi)^{4} \tau_{b}}
$$

$$
\begin{aligned}
& d_{4}=a_{2, b} S_{2}\left[\frac{S_{1}}{\left|E_{\mathrm{K}}\right|}+\frac{3 b_{2, b} \bar{c}_{4}(B \ell)^{4}}{(2 \pi)^{4} \tau_{b}}\right] \\
& d_{5}=\frac{-3 a_{2, b} \bar{c}_{1} g_{R, b} \Gamma_{b}}{2(2 \pi)^{2}} \\
& d_{6}=g_{R, b} S_{1}\left[\frac{S_{1}}{\left|E_{\mathrm{K}}\right|}+\frac{b_{2, b} \bar{c}_{4}(B \ell)^{4}}{(2 \pi)^{4} \tau_{b}}\right]
\end{aligned}
$$




$$
\begin{aligned}
d_{7}= & \frac{g_{R, b} \Gamma_{b}}{2(2 \pi)^{2}}\left[-a_{3, b} \bar{c}_{1}+\frac{\bar{c}_{15} S_{1}(B \ell)^{2}}{(2 \pi)^{2}\left|E_{\mathrm{K}}\right|}\right] \\
d_{8}= & \frac{b_{2, b} \Gamma_{b}}{2(2 \pi)^{2} \tau_{b}}\left[\bar{c}_{0}-a_{1, b} \bar{c}_{1} E_{\mathrm{Na}}+\frac{a_{2, b} \bar{c}_{2} \Gamma_{b}}{[(2 n+1) \ell]^{2}}\right. \\
& \left.+\frac{\bar{c}_{7}}{\tau_{b}}-\frac{\bar{c}_{8} g_{R, b}\left|E_{\mathrm{K}}\right| \Gamma_{R}}{\Gamma_{b}}\right] \\
d_{9}= & \frac{b_{2, b} \bar{c}_{1} g_{R, b} \Gamma_{b}}{2(2 \pi)^{2} \tau_{b}} \\
d_{10}= & \frac{b_{2, b} \Gamma_{b}}{(2 \pi)^{2} \tau_{b}}\left[a_{3, b} \bar{c}_{1}+\frac{\bar{c}_{15} S_{1}(B \ell)^{2}}{(2 \pi)^{2}\left|E_{\mathrm{K}}\right|}\right] \\
d_{11}= & \frac{3 a_{2, b} b_{2, b} \bar{c}_{1} \Gamma_{b}}{2(2 \pi)^{2} \tau_{b}} \\
d_{12}= & \frac{-2 b_{2, b}^{2} \bar{c}_{4} S_{1}(B \ell)^{4}}{(2 \pi)^{4} \tau_{b}^{2}}
\end{aligned}
$$

where $S_{1}, S_{2}$ are dimensionless fractions given in Appendix C and the $\bar{c}_{i}$ symbols denote particular wave-number integrals defined in Appendix E.

Finally, the nonlinear diffusion corrections for the spatially rescaled Fokker-Planck equation (57) are defined by

$$
\begin{aligned}
& \left.\frac{\partial \widehat{P}}{\partial t}\right|_{(2: \text { diffusion })} \\
& \quad=\sum_{b, \vec{J}}\left\{\frac { \partial ^ { 2 } } { \partial \widetilde { V } _ { b , \vec { J } } ^ { 2 } } \left[g_{0}+g_{1} \widetilde{V}_{b, \vec{J}}+g_{2} \widetilde{V}_{b, \vec{J}}^{2}+g_{3} \widetilde{V}_{b, \vec{J}}^{3}\right.\right. \\
& \left.\quad+g_{4} \widetilde{V}_{b, \vec{J}} \widetilde{R}_{b, \vec{J}}\right]+\frac{\partial^{2}}{\partial \widetilde{R}_{b, \vec{J}}^{2}} g_{5} \\
& \left.\quad+\frac{\partial^{2}}{\partial \widetilde{V}_{b, \vec{J}} \partial \widetilde{R}_{b, \vec{J}}}\left[g_{6}+g_{7} \widetilde{V}_{b, \vec{J}}\right]\right\} \widehat{P} \\
& \equiv \sum_{b, \vec{J}}\left\{\frac{\partial^{2}}{\left.\partial \widetilde{V}_{b, \vec{J}}^{2} G_{3}+\frac{\partial^{2}}{\partial \widetilde{R}_{b, \vec{J}}^{2}} g_{5}+\frac{\partial^{2}}{\partial \widetilde{V}_{b, \vec{J}} \partial \widetilde{R}_{b, \vec{J}}} G_{4}\right\} \widehat{P},}\right.
\end{aligned}
$$

with the seven $g_{i}$ coefficients [and SI units] listed below:

$$
\begin{aligned}
& g_{0}=\frac{\Gamma_{b}^{2}}{4(2 \pi)^{2}(B \ell)^{2}}\left[a_{3, b}^{2} \bar{c}_{11}+\frac{1}{2} a_{2, b}^{2} \bar{c}_{12} \Gamma_{b}\right] \quad\left[\mathrm{V}^{2} \mathrm{~s}^{-1}\right], \\
& g_{1}=\frac{3 a_{2, b} a_{3, b} \bar{c}_{11} \Gamma_{b}^{2}}{2(2 \pi)^{2}(B \ell)^{2}} \quad\left[\mathrm{~V} \mathrm{~s}^{-1}\right] \text {, } \\
& g_{2}=\frac{\Gamma_{b}}{2(B \ell)^{2}}\left[\frac{9 a_{2, b}^{2} \bar{c}_{11} \Gamma_{b}}{2(2 \pi)^{2}}-\frac{S_{1}^{2}}{\left|E_{\mathrm{K}}\right|^{2}}\right] \quad\left[\mathrm{s}^{-1}\right], \\
& g_{3}=\frac{3 a_{2, b}(B \ell)^{2} \Gamma_{b}}{2(2 \pi)^{4}\left|E_{\mathrm{K}}\right|}\left[\bar{c}_{15} S_{2}-\frac{\bar{c}_{16}(B \ell)^{2} S_{1}}{(2 \pi)^{2}}\right] \quad\left[\mathrm{V}^{-1} \mathrm{~s}^{-1}\right] \text {, } \\
& g_{4}=\frac{\bar{c}_{15} g_{R, b}(B \ell)^{2} \Gamma_{b} S_{1}}{2(2 \pi)^{4}\left|E_{\mathrm{K}}\right|} \quad\left[\mathrm{V} \mathrm{s}^{-1}\right] \text {, }
\end{aligned}
$$

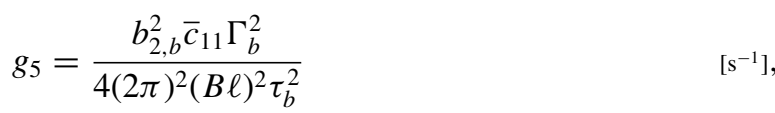

$$
\begin{array}{rlr}
g_{6} & =\frac{-a_{3, b} b_{2, b} \bar{c}_{11} \Gamma_{b}^{2}}{2(2 \pi)^{2}(B \ell)^{2} \tau_{b}} & {\left[\mathrm{~V} \mathrm{~s}^{-1}\right]} \\
g_{7} & =\frac{-3 a_{2, b} b_{2, b} \bar{c}_{11} \Gamma_{b}^{2}}{2(2 \pi)^{2}(B \ell)^{2} \tau_{b}} & {\left[\mathrm{~s}^{-1}\right] .}
\end{array}
$$

In Eqs. (57)-(60), $\widehat{P}$ is the probability distribution function for the macroscopic state variables,

$$
\widetilde{\chi}_{\vec{J}} \in\left\{\widetilde{V}_{b, \vec{J}}, \widetilde{R}_{b, \vec{J}}, \widetilde{Z}_{b, \vec{J}}, \widetilde{\Phi}_{b, \vec{J}}, \widetilde{\Pi}_{b, \vec{J}}, \widetilde{\phi}_{b, \vec{J}}\right\},
$$

where, for example,

$$
\widetilde{V}_{b, \vec{J}}=\frac{2 N+1}{2 n+1} V_{b, \vec{J}}=B^{-1} V_{b, \vec{J}}
$$

gives the voltage for macroscopic cell $\vec{J}$ (whose side length is $L$ ) corresponding to $V_{b, \vec{j}}$, the voltage for microscopic cell $\vec{j}$ (side length $\ell$ ). See Appendices $\mathrm{C}$ and $\mathrm{D}$ for further details.

In the rescaled system, $D_{b}^{\vec{J}} \vec{K}$ and $D_{R}^{\vec{J}} \vec{K}$ represent the discrete Laplacian and biharmonic operators defined on the macroscopic grid in terms of low-frequency spatial modes $\vec{Q}$,

$$
\begin{aligned}
& \sum_{\vec{K}} D_{b}^{\vec{J}} \vec{K} \widetilde{f}_{\vec{K}}(\vec{Q})=-\lambda_{b}(\vec{Q}) \widetilde{f}_{\vec{J}}(\vec{Q}), \\
& \sum_{\vec{K}} D_{R}^{\vec{J} \vec{K}} \widetilde{f}_{\vec{K}}(\vec{Q})=\lambda_{R}(\vec{Q}) \widetilde{f}_{\vec{J}}(\vec{Q}),
\end{aligned}
$$

with eigenfunctions

$$
\widetilde{f}_{\vec{K}}(\vec{Q})=(2 N+1)^{-1} \exp (i L \vec{K} \cdot \vec{Q})
$$

for $\vec{Q} \in \mathscr{R}(N)$, and $L=B \ell$. We also note the Fourier mappings,

$$
\begin{aligned}
& \chi_{\vec{J}}=\sum_{\vec{Q}} \widetilde{f}_{\vec{J}}(\vec{Q}) \chi(\vec{Q}), \\
& \chi(\vec{Q})=\sum_{\vec{J}} \tilde{f}_{\vec{J}}^{*}(\vec{Q}) \chi_{\vec{J}} .
\end{aligned}
$$

Equation (57) encapsulates the essential dynamics of a neural system that has been rescaled from the microscopic level of single spiking neurons to the macroscopic continuum of populations of such neurons. In particular, this formulation demonstrates how intrinsic synaptic and ion-channel noise feeds back into deterministic and stochastic terms at the macroscopic scale.

\section{G. Rescaled stochastic differential equations}

As a final step, we extract the family of macro-scale stochastic DEs (SDEs) implicit in the rescaled Fokker-Planck Eq. (57). These new SDEs are the coarse-scale companions of the fine-scale Langevin equations (25)-(31) listed earlier and are directly applicable to simulation studies of the H. R. Wilson mammalian neuron aggregated to the macro scale.

To establish our indexing convention, we define the spatially rescaled neural state as the 20-element column vector

$$
\begin{aligned}
\widetilde{\vec{\chi}}= & {\left[\widetilde{V}_{e}, \widetilde{V}_{i}, \widetilde{R}_{e}, \widetilde{R}_{i}, \widetilde{\Phi}_{e e}, \widetilde{Z}_{e e}, \widetilde{\Phi}_{e i}, \widetilde{Z}_{e i}, \widetilde{\Phi}_{i e}, \widetilde{Z}_{i e}, \widetilde{\Phi}_{i i}, \widetilde{Z}_{i i},\right.} \\
& \left.\widetilde{\phi}_{e e}, \widetilde{\Pi}_{e e}, \widetilde{\phi}_{e i}, \widetilde{\Pi}_{e i}, \widetilde{\phi}_{i e}, \widetilde{\Pi}_{i e}, \widetilde{\phi}_{i i}, \widetilde{\Pi}_{i i}\right]^{\mathrm{T}} .
\end{aligned}
$$


The reblocked Langevin equations take the general form

$$
\frac{\partial \tilde{\chi}_{j}}{\partial t}=A_{j}+\sum_{k=1}^{20} C_{j k} \xi_{k}(\vec{r}, t),
$$

where $A_{j}$ is the deterministic part and $C_{j k}$ denotes the $(j, k)$ th element of a $20 \times 20$ noise-amplitude matrix $\mathbf{C}$ obeying $\mathbf{C C}^{\mathrm{T}}=\mathbf{B}$. The $\xi_{k}$ (with $\left.k=1: 4,6: 2: 20\right)$ are 12 independent white-noise sources that are $\delta$-correlated in time and 2D space. The nonzero elements of the noise-intensity matrix $\mathbf{B}$ are given by

$$
\begin{aligned}
B_{11} & =-2(B \ell)^{2} G_{3, e}+\Gamma_{e}, & & B_{22}=-2(B \ell)^{2} G_{3, i}+\Gamma_{i}, \\
B_{33} & =-2(B \ell)^{2} g_{5, e}+\Gamma_{R_{e}}, & & B_{44}=-2(B \ell)^{2} g_{5, i}+\Gamma_{R_{i}}, \\
B_{13} & =B_{31}=-(B \ell)^{2} G_{4, e}, & & B_{24}=B_{42}=-(B \ell)^{2} G_{4, i}, \\
B_{66} & =\Gamma_{Z_{e e}}, & & B_{88}=\Gamma_{Z_{e i}}, \\
B_{10,10} & =\Gamma_{Z_{i e}}, & & B_{12,12}=\Gamma_{Z_{i i}}, \\
B_{14,14} & =\Gamma_{\Pi_{e e}}, & & B_{16,16}=\Gamma_{\Pi_{e i}}, \\
B_{18,18} & =\Gamma_{\Pi_{i e}}, & B_{20,20} & =\Gamma_{\Pi_{i i}},
\end{aligned}
$$

where $B$ (italic, no subscripts) is the geometric blocking ratio of Eq. (37), while subscripted $B_{j k}$ denotes the $(j, k)$ th element of intensity matrix $\mathbf{B}$.

Thus, the coarse-grained SDEs can be written

$$
\begin{aligned}
\frac{\partial \widetilde{\chi}_{1}}{\partial t} \equiv & \frac{\partial \widetilde{V}_{e}}{\partial t}=\widetilde{\operatorname{RHS}}(20)_{e}+G_{1, e}+\sum_{k=1}^{20} C_{1 k} \xi_{k}, \\
\frac{\partial \widetilde{\chi}_{2}}{\partial t} \equiv & \frac{\partial \widetilde{V}_{i}}{\partial t}=\widetilde{\operatorname{RHS}}(20)_{i}+G_{1, i}+\sum_{k} C_{2 k} \xi_{k}, \\
\frac{\partial \widetilde{\chi}_{3}}{\partial t} \equiv & \frac{\partial \widetilde{R}_{e}}{\partial t}=\widetilde{\operatorname{RHS}}(21)_{e}+G_{2, e}+\sum_{k} C_{3 k} \xi_{k}, \\
\frac{\partial \widetilde{\chi}_{4}}{\partial t} \equiv & \frac{\partial \widetilde{R}_{i}}{\partial t}=\widetilde{\operatorname{RHS}}(21)_{i}+G_{2, i}+\sum_{k} C_{4 k} \xi_{k}, \\
\frac{\partial}{\partial t} \widetilde{\chi}_{j} \equiv & \frac{d \widetilde{\Phi}_{a b}}{d t}=\widetilde{Z}_{a b}, \\
\frac{\partial}{\partial t} \widetilde{\chi}_{j+1} \equiv & \frac{d \widetilde{Z}_{a b}}{d t} \\
= & -\left(\alpha_{a b}+\beta_{a b}\right) \widetilde{Z}_{a b}-\alpha_{a b} \beta_{a b} \widetilde{\Phi}_{a b} \\
& +\alpha_{a b} \beta_{a b} N_{a b} \psi_{a b}\left(\widetilde{V}_{b}\right) \widetilde{\phi}_{a b}+\sum_{k} C_{j+1, k} \xi_{k}, \\
\frac{\partial}{\partial t} \widetilde{\chi}_{j+8} \equiv & \frac{d \widetilde{\phi}_{a b}}{d t}=\widetilde{\Pi}_{a b}, \\
\widetilde{\chi}_{j+9} \equiv & \frac{\partial \widetilde{\Pi}_{a b}}{\partial t} \\
= & -2 v \Lambda_{a b} \widetilde{\Pi}_{a b}-\left[\left(v \Lambda_{a b}\right)^{2}-(v \vec{\nabla})^{2}\right] \widetilde{\phi}_{a b} S_{a}\left(\widetilde{V}_{a}-V_{a}^{\mathrm{rest}}\right)+\sum_{k} C_{j+9, k} \xi_{k}, \\
& +(v)
\end{aligned}
$$

where label $a b$ cycles through $\{e e, e i, i e, i i\}$ as index $j$ steps through $\{5,7,9,11\}$ in Eqs (68)-(71).

The slow-soma voltage $\widetilde{\widetilde{V}}_{b}$ appearing in Eq. (69) is computed from the moving-average differential equation (23), but expressed at the reblocked scale,

$$
\frac{d \widetilde{V}_{b}}{d t}=-\frac{1}{T}\left[\widetilde{\widetilde{V}}_{b}(t)-\widetilde{V}_{b}(t)\right]
$$

\section{DEMONSTRATION OF METHOD: REBLOCKED WILSON SPIKING NEURON}

Although the major aim of this paper is to introduce a systematic algorithm for spatial rescaling for a cortical network, as a first-order demonstration of the method it is useful to examine the spiking properties of the reblocked H. R. Wilson neuron for the highly simplified limiting case of negligible noise and fully suppressed chemical synapses. In this deterministic limit, the neuron is embedded in a network of identical spiking neurons that are coupled by gap junctions only.

For this simplified test case, the 20 stochastic partial DEs (64)-(71) reduce to a single pair of deterministic partial DEs for $\left(\widetilde{V}_{b}, \widetilde{R}_{b}\right) \rightarrow(\widetilde{V}, \widetilde{R})$, the reblocked voltage and recovery variables within a single population: Dropping the noise terms in Eqs (64) and (66) and setting $\Phi_{a b}=0$ (i.e., no synaptic inputs) in Eq. (20) gives

$$
\begin{aligned}
\frac{\partial \widetilde{V}}{\partial t}= & -g(\widetilde{V})\left(\widetilde{V}-E_{\mathrm{Na}}\right)-g_{R} \widetilde{R} \cdot\left(\widetilde{V}-E_{\mathrm{K}}\right)+I^{\mathrm{dc}} / C \\
& +D_{b} \nabla^{2} \widetilde{V}+G_{1}(\widetilde{V}, \widetilde{R})
\end{aligned}
$$

$$
\frac{\partial \widetilde{R}}{\partial t}=-\frac{1}{\tau_{b}}\left[\widetilde{R}-R_{\infty}(\widetilde{V})\right]+\frac{D_{R} \nabla^{4} \widetilde{V}}{\tau_{b}}+G_{2}(\widetilde{V}, \widetilde{R}),
$$

where $G_{1}, G_{2}$ are the reblocking nonlinear drift corrections from Eq. (59) defined in terms of its $12 d_{j}$ coefficients. Since we are considering the small-noise limit (i.e., $\Gamma_{b}=\Gamma_{R} \approx 0$ ), most of these coefficients become zero, and the $G_{1}, G_{2}$ corrections reduce to

$$
\begin{aligned}
& G_{1}=d_{3} \widetilde{V}^{3}+d_{4} \widetilde{V}^{4}+d_{6} \widetilde{V}^{2} \widetilde{R}, \\
& G_{2}=d_{12} \widetilde{V}^{3},
\end{aligned}
$$

with

$$
\begin{aligned}
d_{3} & =\frac{a_{3} S_{1}^{2}}{\left|E_{\mathrm{K}}\right|}+\left[2 a_{3} \bar{c}_{4}-\frac{\bar{c}_{5}}{\left|E_{\mathrm{K}}\right|}\right] \frac{b_{2} S_{1}(B \ell)^{4}}{(2 \pi)^{4} \tau_{b}}, \\
d_{4} & =a_{2} S_{2}\left[\frac{S_{1}}{\left|E_{\mathrm{K}}\right|}+\frac{3 b_{2} \bar{c}_{4}(B \ell)^{4}}{(2 \pi)^{4} \tau_{b}}\right], \\
d_{6} & =g_{R} S_{1}\left[\frac{S_{1}}{\left|E_{\mathrm{K}}\right|}+\frac{b_{2} \bar{c}_{4}(B \ell)^{4}}{(2 \pi)^{4} \tau_{b}}\right], \\
d_{12} & =\frac{-2 b_{2}^{2} \bar{c}_{4} S_{1}(B \ell)^{4}}{(2 \pi)^{4} \tau_{b}^{2}} .
\end{aligned}
$$

For our demonstration, we set the microcell dimension at $\ell=10 \mu \mathrm{m}$ and the blocking ratio at $B=100$. The $\bar{c}_{4}$ and $\bar{c}_{5}$ elements appearing in the four $d$ coefficients above are the small- $\vec{Q}$ wave-number integrals (see Appendix E)

$$
\begin{aligned}
\bar{c}_{4} & =\iint \frac{d \vec{Q}_{1} d \vec{Q}_{2}}{\Lambda_{R}\left(\vec{Q}_{1}+\vec{Q}_{2}\right)}, \\
\bar{c}_{5} & =\iint \frac{\Lambda_{b}\left(\vec{Q}_{1}+\vec{Q}_{2}\right) d \vec{Q}_{1} d \vec{Q}_{2}}{\Lambda_{R}\left(\vec{Q}_{1}+\vec{Q}_{2}\right)},
\end{aligned}
$$


which are to be evaluated subject to the restriction $\left(\vec{Q}_{1}+\right.$ $\left.\vec{Q}_{2}\right)=\vec{q}$. The required $\Lambda_{b}$ and $\Lambda_{R}$ definitions are given in (B2b) and (B2c), respectively,

$$
\Lambda_{b}(\vec{q})=\lambda_{b}(\vec{q})+a_{0}, \quad \Lambda_{R}(\vec{q})=\lambda_{R}(\vec{q})+b_{1} / \tau_{b},
$$

with eigenvalues $\lambda_{b}(\vec{q}), \lambda_{R}(\vec{q})$ defined previously in Eqs. (34),

$$
\begin{gathered}
\lambda_{b}(\vec{q})=\frac{4 D_{b}}{\ell^{2}}\left[\sin ^{2}\left(q_{x} \ell / 2\right)+\sin ^{2}\left(q_{y} \ell / 2\right)\right], \\
\lambda_{R}(\vec{q})=\frac{16 D_{R}}{\ell^{4} \tau_{b}}\left[\sin ^{2}\left(q_{x} \ell / 2\right)+\sin ^{2}\left(q_{y} \ell / 2\right)\right]^{2} .
\end{gathered}
$$

While the voltage-diffusion coefficient $D_{b}$ can be estimated from the measured properties and abundance of electrical gap junctions [see Eq. (18)], the value of the diffusive-recovery coefficient $D_{R}$ is much more uncertain. However, by requiring that the projection operators of Appendix B (specifically the auxiliary operators $b_{1_{+}}, b_{1_{-}}, b_{2_{+}}, b_{2_{-}}$) remain purely real, we can write down an expression for the upper bound on $D_{R}$. Examining (B11b) and (B2a), in order for $\delta(\vec{q})$ to be real we require

$$
\Lambda_{b}^{2}(\vec{q}) \geqslant 4 g_{R}\left|E_{\mathrm{K}}\right| \Lambda_{R}(\vec{q}) .
$$

Substituting Eqs. (75) and (76) and isolating $D_{R}$ leads to the inequality

$$
D_{R} \leqslant \frac{\ell^{4} \tau_{b}}{64 \sin ^{4}(q \ell / 2)}\left\{\frac{\left[\left(8 D_{b} / \ell^{2}\right) \sin ^{2}(q \ell / 2)+a_{0}\right]^{2}}{4 g_{R}\left|E_{\mathrm{K}}\right|}-b_{1} / \tau_{b}\right\},
$$

where we have set $q_{x}=q_{y}=q$.

Defining $D_{R}^{\max }$ to be the upper bound of inequality (77), we plot $D_{R}^{\max }$ as a function of gap-junction diffusivity $D_{b}$ in Fig. 5 . The dashed and solid curves plotted there correspond to the two alternative $D_{R}$ upper bounds implied by the inner (small wave number) and outer (large wave number) Fourier limits

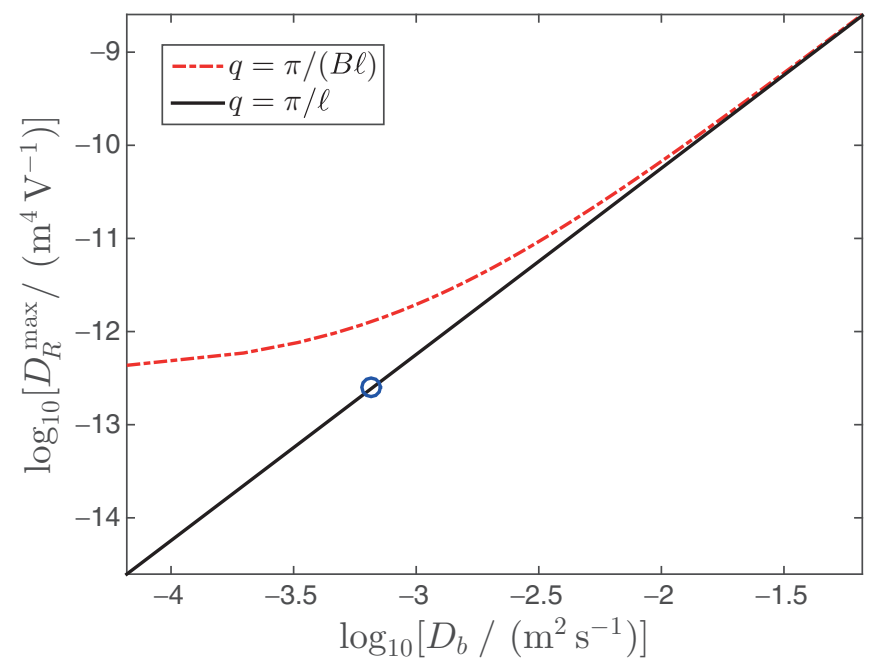

FIG. 5. Upper limits for recovery diffusivity $D_{R}$ as a function of voltage diffusion coefficient $D_{b}$. The dashed curve corresponds to the small wave-number limit $q=Q^{\max }=\pi /(B \ell)$, where $B=100$ is the blocking ratio, while the solid line applies the more conservative limit $q=q^{\max }=\pi / \ell$. The circled point shows the selected operating point $D_{b}=6.60 \times 10^{-4} \mathrm{~m}^{2} / \mathrm{s}, D_{R}=2.47 \times 10^{-13} \mathrm{~m}^{4} / \mathrm{V}$. in Fig. 4(c): the inner boundary at $q=Q^{\max }=\pi / L$ and the outer boundary at $q=q^{\max }=\pi / \ell$. The latter sets the more conservative (lower) limit on $D_{R}^{\max }$ since it ensures that the auxiliary operators remain real over the entire wave-number domain of interest. In order to constrain the model (and in the absence of any experimental knowledge of $D_{R}$ ), we estimate $D_{b}$, then set the recovery diffusivity at its maximum allowable value $D_{R}=D_{R}^{\max }$ as determined from the solid curve in Fig. 5 .

We estimate voltage diffusivity $D_{b}$ by linking the present spiking model with an earlier mean-field cortical model for gap-junction connectivity [41]. Working from measurements by Fukuda et al. [48] of gap-junction connectivity for inhibitory neurons in cat visual cortex, Steyn-Ross et al. [41] established a gap-junction coupling strength $D_{2}$ defined as the areal region of influence of a so-called "Fukuda cell"; values for $D_{2}$ fell in the range $0.1 \leqslant D_{2} \leqslant 0.6 \mathrm{~cm}^{2}$. The ratio $D_{2} / T$, where $T=40 \mathrm{~ms}$ is the soma time constant, defined a meanfield coefficient of voltage diffusion which covers the range

$$
2.5 \times 10^{-4} \leqslant D_{2} / T \leqslant 15 \times 10^{-4} \mathrm{~m}^{2} / \mathrm{s} .
$$

We set $D_{b}=6.60 \times 10^{-4} \mathrm{~m}^{2} / \mathrm{s}$ (towards the bottom end of the $D_{2} / T$ range), and $D_{R}=2.47 \times 10^{-13} \mathrm{~m}^{4} / \mathrm{V}$ [corresponding to the $D_{R}^{\max }$ upper bound from inequality (77); see circled point in Fig. 5], having established (via numerical simulations, not shown here) that lower $\left(D_{b}, D_{R}\right)$ diffusivity values do not support spiking dynamics in the reblocked Wilson neuron.

From the Eq. (18) definition of voltage diffusivity, the assumed value for $D_{b}$ implies an effective gap-junction (GJ) conductance of $g^{\text {gap }}=D_{b} C / \ell^{2}=6.60 \times 10^{4} \mathrm{~S} / \mathrm{m}^{2}$. This value is comparable with-albeit larger than-quoted values in the GJ literature. In their numerical modeling studies, Hand and Griffith [49] take normal GJ conductance of cardiac tissue to be $6660 \mathrm{~S} / \mathrm{m}^{2}$; meanwhile, Martinez-Wittinghan et al. [50] measured the connexon-50 (Cx50) GJ conductance of healthy lens tissue to be of order $10^{4} \mathrm{~S} / \mathrm{m}^{2}$. We consider that using a somewhat higher value for neuron-to-neuron conductance here is not unreasonable given the expected boost from parallel conductance paths via the surrounding neuroglial scaffolding that contacts and services the neurons [51].

Having set the $\left(D_{b}, D_{R}\right)$ diffusivities, we evaluate the $\bar{c}_{4}$ and $\bar{c}_{5}$ wave-number integrals using Monte Carlo integration $\left(2.5 \times 10^{6}\right.$ samples $)$, obtaining

$$
\begin{aligned}
& \bar{c}_{4}=(6.337 \pm 0.003) \times 10^{10} \mathrm{~V} \mathrm{~s} / \mathrm{m}^{4}, \\
& \bar{c}_{5}=(1.916 \pm 0.001) \times 10^{15} \mathrm{~V} / \mathrm{m}^{4},
\end{aligned}
$$

and finally compute the $G_{1}, G_{2}$ blocking correction terms of Eqs. (73) and (74). We then locate the steady states, and their linear stability, for the homogeneous reblocked Wilson neuron, and track how stability changes as a function of $I^{\mathrm{dc}}$ current drive. Onset of spiking is predicted at the point at which the dominant eigenvalue has a real part that changes sign from negative (stable steady state) to positive (unstable). For the original H. R. Wilson neuron, this bifurcation occurs via a saddle-node annihilation (dominant eigenvalue is real), but for the reblocked neuron, we find that the dominant eigenvalue is complex, with loss of stability occurring via a Hopf transition. Unexpectedly, the blocking procedure has transformed a type 1 integrator neuron into type 2 resonator whose firing-rate curve shows an abrupt ("discontinuous") 


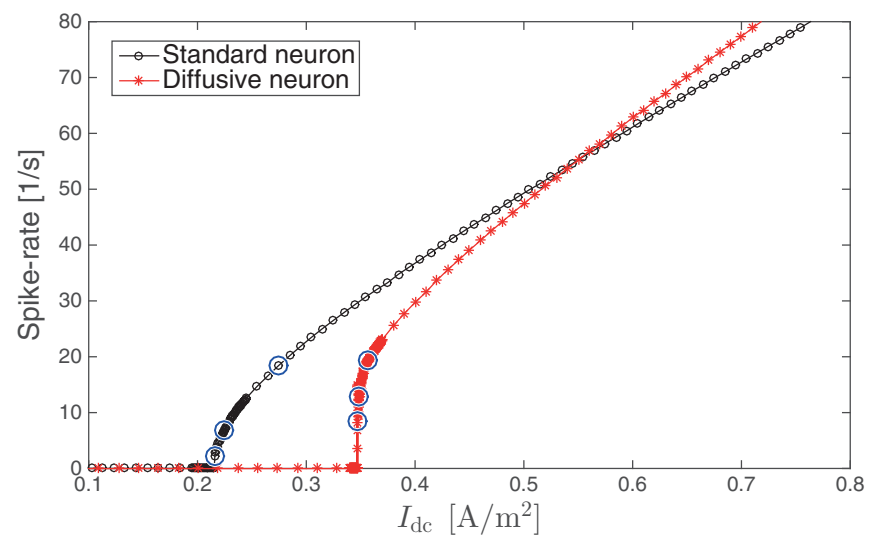

FIG. 6. Current-to-firing rate transfer functions for (a) standard Wilson integrator neuron [Eqs. $(1,2)]$ (small black circles) and (b) reblocked diffusive Wilson neuron [Eqs. (73) and (74)] (red asterisks). The transfer function for the standard neuron increases smoothly from zero, whereas the reblocked neuron shows a discontinuity with near-vertical takeoff above threshold. The threshold currents are $I_{\mathrm{dc}}^{\text {crit }} /\left(\mathrm{A} \mathrm{m}^{-2}\right) \approx 0.21475$ and 0.34652 for the standard and reblocked neurons, respectively. The large blue circles mark the $I_{\mathrm{dc}}$ drive-current values selected for the six time series displayed in Fig. 7.

spiking onset. The comparison between the standard and reblocked transfer functions is illustrated in Fig. 6.

The firing patterns just above threshold shown in Fig. 7 clearly show the change in dynamics wrought by the reblocking: Whereas the standard neuron can fire arbitrarily slowly, the diffusive neuron has a preferred firing rate, but just the above critical current, the firings are chaotic and intermittent, being interspersed with variable intervals of subthreshold ringing. We emphasize that this intermittency is entirely deterministic: No noise has been included in the simulations.

\section{DISCUSSION}

In this paper we have addressed a long-standing unresolved issue in mean-field modeling of the cortex: How does one construct a spatial mapping that permits a consistent scaling from micro-level single-neuron dynamics to a macro-level description for a neural population? We have presented a stringent coarse-graining algorithm that derives equations for "specific" neural activity, that is, the behavior of a neuron sampled at random from the population, yet subject to noisy neural inputs from other members of that population. This regridding approach stands in marked contrast to standard mean-field averages which necessarily neglect correlations in neuronal fluctuations. We believe that our coarse-grained equations are a closer depiction of the actual population-level "true field."

The coarse-graining procedure applies an adiabatic elimination of short-wavelength spatial scales [29,31,32], which was shown by SR\&G [33] to be equivalent to the generalized path (functional) integration performed by $\mathrm{K}$. Wilson in his renormalization group solution to the Ising ferromagnet [30]. This equivalence gives us confidence that the coarse-graining formalism provides a mathematically systematic algorithm for the spatial regridding of the cortex.

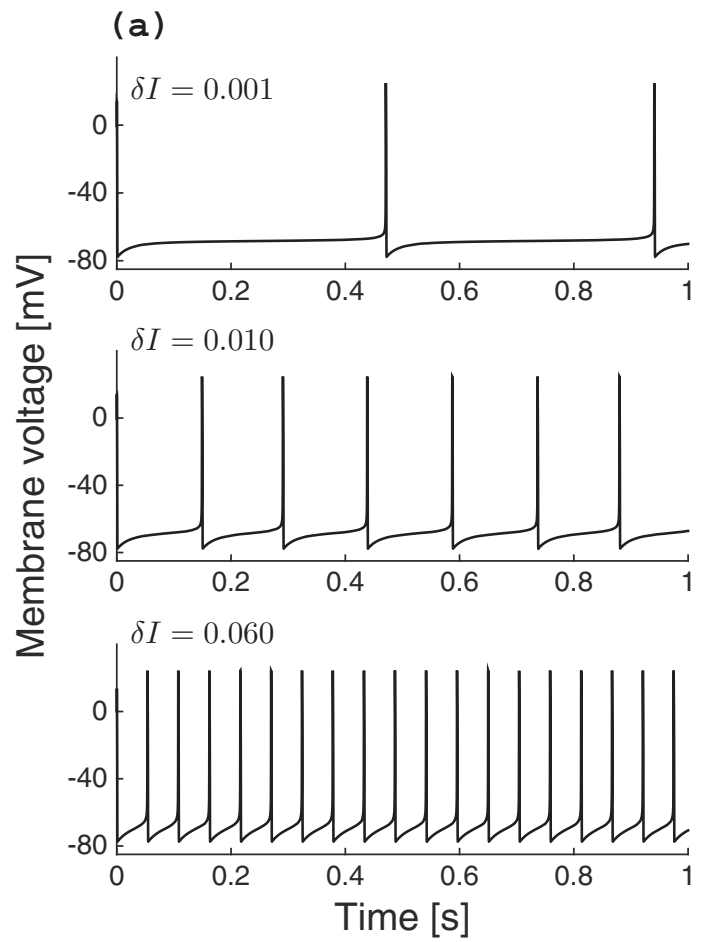

\section{(b)}
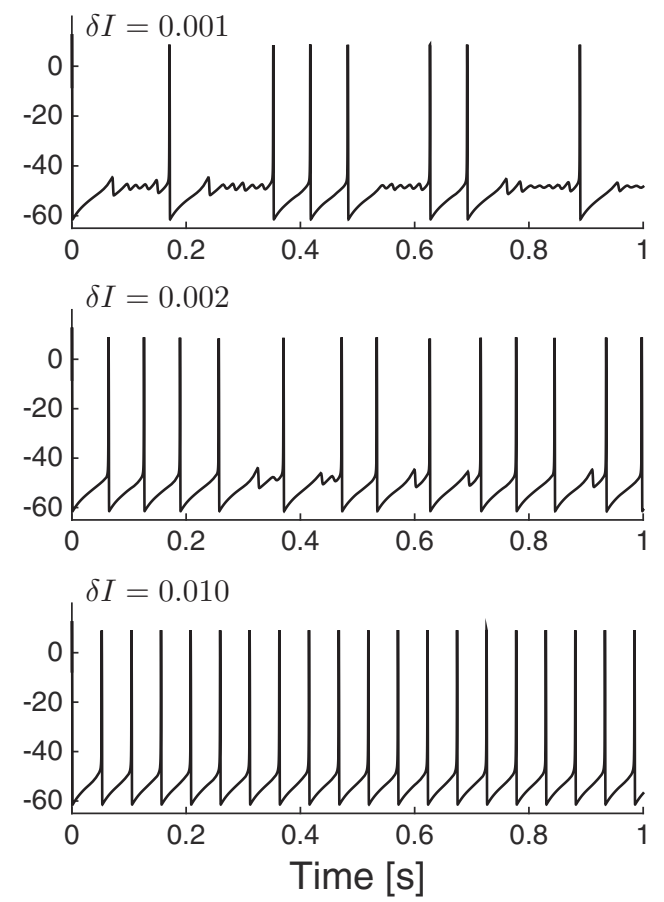

FIG. 7. Comparison between firing patterns for (a) standard Wilson integrator neuron (left-hand panels) and (b) reblocked diffusive Wilson neuron (right-hand panels) for three settings of $I_{\mathrm{dc}}$ drive current just above threshold: $I_{\mathrm{dc}}=I_{\mathrm{dc}}^{\text {crit }}+\delta I$. For the reblocked neuron in (b), firing onset is abrupt and chaotic, and exhibits damped nonlinear oscillations between firing events, whereas for the the standard integrator, firing onset is smooth and graduated. Evidently, reblocking has transformed the type 1 integrator into a type 2 resonator neuron. 
However, we need to acknowledge a significant simplification we have made in our cortical model: We have assumed that all synaptic interactions-both GJ mediated and chemical-are purely local. This is manifestly not the case in a real cortex with its millions of long-range whitematter anatomical connections joining distant cortical areas. Despite this limitation, the reblocking technique provides a way forward for deriving coarse-grained approximations of neuronal dynamics on a structureless homogeneous cortex.

Our spiking-neuron model is based on H. R Wilson's two-variable $(V, R)$ voltage-recovery reduction of the standard four-variable $\mathrm{HH}$ equations [4,5]. An enhancement to this model is the introduction of an explicit biharmonic spatial dependence for the recovery variable $R$. This step was central to our method for both biophysical and technical reasons.

(i) $R$ is a key component of the spiking model since it represents the activation of $\mathrm{K}^{+}$outflow (and simultaneous inactivation of $\mathrm{Na}^{+}$inflow), leading to the quelling of the action-potential spike and subsequent recovery of membrane voltage towards its resting value. Since $R$ can be regarded as a proxy for the probability of K-channel opening, the spatial dependence of this "probability" variable is likely to be important, but its spatial characteristics have yet to be established. Nevertheless, in Sec. II A we developed a plausibility argument justifying our inclusion of a $\nabla^{4} V_{b}$ term in the $\partial R / \partial t$ equation of motion.

(ii) The elimination of short-wavelength (high- $\vec{q}$ ) modes can only succeed if the perturbative expansion of Sec. IIE converges. This mandates a careful choice for the $L_{1}$ projection operator. We are motivated to choose an $L_{1}$ ansatz which is based on the classic Ornstein-Uhlenbeck (OU) form since this is arguably the most thoroughly studied stochastic system, it has a well-known stationary solution, and was used by SR\&G [33] in their adiabatic treatment of the LandauGinzburg model.

Here we need to consider the coupling between variables $V$ and $R$, so we adopt the two-variable OU form, referred to as Kramer's equation. After careful consideration we determined that the biharmonic form for $R$ is the simplest representation that provides adiabatic closure within the context of the Kramer ansatz.

The elimination procedure generates a multiplicity of technically challenging calculations. To systematize the calculations, we made extensive use of boson creation-annihilation operators. This mathematical convenience is permitted because of a formal equivalence between the solutions of Kramer's equation and the solutions of the harmonic oscillator. In Appendix B we demonstrate that this allows us to identify a "vacuum state" for the cortex, but the potential biophysical significance of such a ground state is unclear.

As was the case with K. Wilson's treatment of the Ising ferromagnet, our method is predicated on the convergence of a perturbative expansion via nearest-neighbor diffusive coupling.

In our case, this requires a sufficient GJ diffusion $D_{b}>0$ such that the Laplacian terms of $L_{1}$ dominate the denominator of Eq. (50d). In Appendix F, we demonstrate that $L_{1}$ dominance can be achieved provided that the voltage-ratio coupling coefficient between GJ coupled pairs of neurons exceeds $\sim 1 \%$. While GJ connections between excitatory neurons seem to be very rare, electrical connections between pairs of inhibitory neurons are ubiquitous throughout the cortex [52,53], yet their coupling tends to be rather weak. Wang et al. [53] report coupling coefficients for inhibitory-inhibitory pairings ranging from $6 \%$ to $17 \%$, well above the $1 \%$ minimum we require for convergence of our perturbative theory for spatial reblocking.

Gap-junction diffusion might provide a natural mechanism for the formation of "cortical columns." First proposed by Mountcastle [54] as "an elementary unit of organization in the somatic cortex," neurons within these functional units are activated by the same stimulus and express the same collective firing properties. The existence of such columns has been supported by many [55-57]; however, their purpose has also been questioned [58] because of the high level of variability of these clusters within the same species. We hypothesize that within the constraints imposed by the limits of convergence for spatial coarse graining, "columns" of functionally connected neurons could emerge spontaneously from a homogeneous grid of spiking neurons devoid of any directed connections. The adiabatic reblocking approach described in the present paper might provide a way to test this hypothesis both analytically and numerically.

The development of the micro-scale model of a diffusively coupled network of spiking neurons - as detailed in Sec. II A-will trigger a full investigation of the ungridded fine-grain dynamics to inform future research.

We close by listing some immediate and potential neuralfield applications of the adiabatic H. R. Wilson "true-field" equations.

(a) In Sec. III we extracted the macrocolumn-scale currentto-firing-rate transfer function for a population of diffusively coupled identical type 1 (integrator) cortical neurons in the rather artificial limit of negligible noise and disabled chemical synapses. As immediate next steps, we should relax these restrictions in stages by (i) introducing distinct excitatory and inhibitory neural types; (ii) enabling synaptic and ionchannel noises; (iii) enabling the wave-equation fluxes of spike activity in both deterministic and fully stochastic limits. In addition, it would be a straightforward to generalize the true-field approach to populations of diffusively coupled type 2 (resonator) neurons by replacing the quadratic $R_{\infty}$ polynomial in Eq. (2) (describing a type 1 membrane) with a linear form [4].

(b) Mean-field models have already been been applied with some success to a range of neurotransmitter-modulated phase transitions such as natural sleep [21,22,59-61], anesthesia [19,62-72], and seizure [73-78]; for example, anesthetic induction is modeled by increasing the area of the inhibitory postsynaptic potential (PSP) in a drug-dependent manner. Similar rate-constant modulations of PSP could be applied to the true-field spiking equations to investigate changes in spiking rhythms near phase-transition tipping points.

(c) Because the true-field equations are explicitly dependent on the blocking ratio $B$, one could analyze how fluctuation spectra and spiking avalanche statistics vary with area scaling. As a final conjecture, we suggest that it might be possible to establish renormalization-group-like scaling laws - and their associated critical exponents-for close approach to cortical transition points separating distinct states of vigilance. 


\section{ACKNOWLEDGMENT}

We thank Professor Hugh Wilson for providing background details about the foundation and construction of his spikingneuron model.

\section{APPENDIX A: REBLOCKING CORRECTIONS EXPRESSED AS VACUUM EXPECTATIONS}

Here we show how Eq. (51) can be expressed in terms of expectation values. Inserting definition $L_{3}=\mathscr{L}_{3}(\vec{q}, \vec{Q})+$ $L_{3}(\vec{Q})$ of Eq. (43) into (51) gives

$$
\begin{aligned}
\frac{\partial v}{\partial t}= & \mathscr{P}\left[\mathscr{L}_{3}+L_{3}(\vec{Q})\right] v(t) \\
& -\mathscr{P}\left[\mathscr{L}_{3}+L_{3}(\vec{Q})\right] L_{1}^{-1}\left\{L_{2}+(1-\mathscr{P})\right. \\
& \left.\times\left[\mathscr{L}_{3}+L_{3}(\vec{Q})\right]\right\} v(t) .
\end{aligned}
$$

Recalling from Eq. (52) that

$$
v(t)=\mathscr{P} P(\chi, t)=P_{s} \widehat{P}(\chi(\vec{Q}, t)),
$$

with projection operator $\mathscr{P}$ defined in (46), the first term on the RHS of (A1) becomes

$$
P_{s} \int\left[\mathscr{L}_{3}+L_{3}(\vec{Q})\right] P_{s} d \chi(\vec{q}) \widehat{P}=P_{s}\left[\left\langle\mathscr{L}_{3}\right\rangle+\left\langle L_{3}(\vec{Q})\right\rangle\right] \widehat{P},
$$

where $\langle\cdot\rangle$ denotes the stationary expectation over $\vec{q}$ space; e.g., for arbitrary function $f[\chi(\vec{q})]$,

$$
\langle f[\chi(\vec{q})]\rangle \equiv \int f[\chi(\vec{q})] P_{s}(\chi(\vec{q})) d \chi(\vec{q}) .
$$

In the second term on the RHS of (A1), we note that operator $\mathscr{P}$ commutes with both $L_{3}(\vec{Q})$ and $L_{1}^{-1}$; thus,

$$
\begin{aligned}
& \mathscr{P} L_{3}(\vec{Q}) L_{1}^{-1}\left\{L_{2}+(1-\mathscr{P})\left[\mathscr{L}_{3}+L_{3}(\vec{Q})\right]\right\} v(t) \\
& \quad=L_{3}(\vec{Q}) L_{1}^{-1}\left\{\mathscr{P} L_{2}+\mathscr{P}(1-\mathscr{P})\left[\mathscr{L}_{3}+L_{3}(\vec{Q})\right]\right\} v(t), \\
& \quad=0,
\end{aligned}
$$

which follows because $\mathscr{P} L_{2}=0$ [from Eq. (47c)]; and $\mathscr{P}(1-$ $\mathscr{P})=\mathscr{P}-\mathscr{P}^{2}=0$ because the $\mathscr{P}$ projection operator is idempotent. Furthermore,

$$
\begin{aligned}
(1-\mathscr{P}) L_{3}(\vec{Q}) v(t) & =(1-\mathscr{P}) L_{3}(\vec{Q}) \mathscr{P} P \\
& =(1-\mathscr{P}) \mathscr{P} L_{3}(\vec{Q}) P \\
& =0,
\end{aligned}
$$

so the second term on the RHS of (A1) simplifies to

$$
\begin{aligned}
\mathscr{P} \mathscr{L}_{3} L_{1}^{-1}\left[L_{2}+(1-\mathscr{P}) \mathscr{L}_{3}\right] v(t) \\
=P_{s}\left\langle\mathscr{L}_{3} L_{1}^{-1}\left[L_{2}+(1-\mathscr{P}) \mathscr{L}_{3}\right]\right\rangle \widehat{P} .
\end{aligned}
$$

Thus, (A1) becomes

$$
\begin{aligned}
\frac{d v}{d t}= & P_{s} \frac{\partial \widehat{P}}{\partial t} \\
= & P_{s}\left\{\left\langle\mathscr{L}_{3}\right\rangle+\left\langle L_{3}(\vec{Q})\right\rangle\right. \\
& \left.-\left\langle\mathscr{L}_{3} L_{1}^{-1}\left[L_{2}+(1-\mathscr{P}) \mathscr{L}_{3}\right]\right\rangle\right\} \widehat{P}
\end{aligned}
$$

and we obtain the rescaled Fokker-Planck equation in $\vec{Q}$ space,

$$
\begin{aligned}
\frac{\partial \widehat{P}}{\partial t}= & \left\{\left\langle L_{3}(\vec{Q})\right\rangle+\left\langle\mathscr{L}_{3}\right\rangle-\left\langle\mathscr{L}_{3} L_{1}^{-1} L_{2}\right\rangle\right. \\
& \left.-\left\langle\mathscr{L}_{3} L_{1}^{-1}(1-\mathscr{P}) \mathscr{L}_{3}\right\rangle\right\} \widehat{P} .
\end{aligned}
$$

The leading $\left\langle L_{3}(\vec{Q})\right\rangle$ term represents an exact version of the original Fokker-Planck equation in $\vec{Q}$ space. The remaining terms are corrections arising from nonlinear interactions. Explicit evaluation of the expectation values in (A3) follows most directly via the operator formalism described below.

\section{APPENDIX B: OPERATOR FORMALISM}

Elimination of high- $\vec{q}$ modes proceeds by applying the projection operator $\mathscr{P}$ of Eq. (46) constructed from the stationary solution of $L_{1}$,

$$
L_{1} P_{s}=0 .
$$

Working from Eq. (39), we express $L_{1}$ as

$$
L_{1}=L_{1}(V, R)+L_{1}(Z, \Phi)+L_{1}(\Pi, \phi),
$$

where

$$
\begin{gathered}
L_{1}(V, R)=\sum_{b} \sum_{\vec{q}}\left\{\frac{\partial}{\partial V_{b}(\vec{q})} \Lambda_{b}(\vec{q}) V_{b}(\vec{q})+\frac{\partial}{\partial V_{b}(\vec{q})} g_{0, R} R_{b}(\vec{q})\right. \\
\left.-\frac{\partial}{\partial R_{b}(\vec{q})} \Lambda_{R}(\vec{q}) V_{b}(\vec{q})+\frac{\Gamma_{b}}{2 \ell^{2}} \frac{\partial^{2}}{\partial V_{b}(\vec{q}) V_{b}(-\vec{q})}\right\},
\end{gathered}
$$

with

$$
g_{0, R}=g_{R, b}\left|E_{\mathrm{K}}\right|,
$$

$$
\Lambda_{b}(\vec{q})=\lambda_{b}(\vec{q})+a_{0, b},
$$

$$
\Lambda_{R}(\vec{q})=\lambda_{R}(\vec{q})+b_{1, b} / \tau_{b},
$$

and

$$
\begin{aligned}
L_{1}(Z, \Phi)= & \sum_{a, b} \sum_{\vec{q}}\left\{-\frac{\partial}{\partial \Phi_{a b}(\vec{q})} Z_{a b}(\vec{q})\right. \\
& +\frac{\partial}{\partial Z_{a b}(\vec{q})}\left[\bar{a}_{2} Z_{a b}(\vec{q})+\bar{a}_{3} \Phi_{a b}(\vec{q})\right] \\
& \left.+\frac{\Gamma_{Z}}{2 \ell^{2}} \frac{\partial^{2}}{\partial Z_{a b}(\vec{q}) Z_{a b}(-\vec{q})}\right\},
\end{aligned}
$$

with

$$
\bar{a}_{2}=\alpha_{a b}+\beta_{a b}, \quad \bar{a}_{3}=\alpha_{a b} \beta_{a b},
$$

and

$$
\begin{aligned}
L_{1}(\Pi, \phi)= & \sum_{a, b} \sum_{\vec{q}}\left\{-\frac{\partial}{\partial \phi_{a b}(\vec{q})} \Pi_{a b}(\vec{q})\right. \\
& +\frac{\partial}{\partial \Pi_{a b}(\vec{q})}\left[2 v \Lambda_{a b} \Pi_{a b}(\vec{q})+\bar{a}_{4} \phi_{a b}(\vec{q})\right] \\
& \left.+\frac{\Gamma_{\Pi}}{2 \ell^{2}} \frac{\partial^{2}}{\partial \Pi_{a b}(\vec{q}) \Pi_{a b}(-\vec{q})}\right\},
\end{aligned}
$$


with

$$
\bar{a}_{4}=+D_{\phi} \lambda_{\phi}(\vec{q})+\left(v \Lambda_{a b}\right)^{2} .
$$

When expressed in this form, $L_{1}$ satisfies

$$
L_{1} P_{s}=0 \text {, }
$$

where the stationary probability distribution is expressed as a product,

$$
P_{s}=P_{s}(V, R) P_{s}(Z, \Phi) P_{s}(\Pi, \phi) .
$$

We demonstrate assertion (B5) by first considering $L_{1}(V, R)$. We make the following identifications:

$$
\begin{aligned}
V_{b}(\vec{q}) & =\sqrt{\frac{\Gamma_{b}}{\Lambda_{b}(\vec{q})} \frac{y_{b}(\vec{q})}{\ell},} \\
R_{b}(\vec{q}) & =\sqrt{\frac{\Gamma_{b} \Lambda_{R}(\vec{q})}{g_{0, R} \Lambda_{b}(\vec{q})}} \frac{x_{b}(\vec{q})}{\ell}, \\
\frac{\partial}{\partial V_{b}(\vec{q})} & =\ell \sqrt{\frac{\Lambda_{b}(\vec{q})}{\Gamma_{b}} \frac{\partial}{\partial y_{b}(\vec{q})},} \\
\frac{\partial}{\partial R_{b}(\vec{q})} & =\ell \sqrt{\frac{g_{0, R} \Lambda_{b}(\vec{q})}{\Gamma_{b} \Lambda_{R}(\vec{q})}} \frac{\partial}{\partial x_{b}(\vec{q})} .
\end{aligned}
$$

Then (B1) can be rewritten as

$$
\begin{aligned}
\sum_{b} \sum_{\vec{q}} & \left\{\Lambda_{b}(\vec{q})\left[\frac{\partial}{\partial y_{b}(\vec{q})} y_{b}(\vec{q})+\frac{1}{2} \frac{\partial^{2}}{\partial y_{b}(\vec{q}) y_{b}(-\vec{q})}\right]\right. \\
& +\sqrt{g_{0, R} \Lambda_{R}(\vec{q})} \frac{\partial}{\partial y_{b}(\vec{q})} x_{b}(\vec{q}) \\
& \left.-\sqrt{g_{0, R} \Lambda_{R}(\vec{q})} \frac{\partial}{\partial x_{b}(\vec{q})} y_{b}(\vec{q})\right\}=L_{1}(V, R) .
\end{aligned}
$$

This matches the form of Kramer's equation, which satisfies

$$
L_{1}(V, R) P_{s}(V, R)=0,
$$

where

$$
\begin{aligned}
P_{s}(V, R)= & \mathscr{N} \exp \left[-2 y_{b}(\vec{q}) y_{b}(-\vec{q})\right] \\
& \times \exp \left[-2 x_{b}(\vec{q}) x_{b}(-\vec{q})\right] \equiv|0\rangle_{V, R},
\end{aligned}
$$

with $\mathscr{N}$ being an appropriate normalization for the stationary probability distribution.

The $y$ and $\frac{\partial}{\partial y}$ components of $L_{1}(V, R)$ define a conjugate pair for membrane voltage $V$ that are analogous to position and momentum coordinates for a quantum harmonic oscillator; similarly, $x$ and $\frac{\partial}{\partial x}$ are the corresponding conjugate pair for membrane recovery $R$. We now introduce bosonic creation operators

$$
A_{b}^{\dagger}(\vec{q}), \quad A_{R}^{\dagger}(\vec{q})
$$

and annihilation operators

$$
A_{b}(\vec{q}), \quad A_{R}(\vec{q}),
$$

where $A_{R} \in\left\{A_{R_{e}}, A_{R_{i}}\right\}$, and

$$
\begin{aligned}
y_{b}(\vec{q}) & =\left[A_{b}^{\dagger}(\vec{q})+A_{b}(-\vec{q})\right] / \sqrt{2}, \\
x_{b}(\vec{q}) & =\left[A_{R}^{\dagger}(\vec{q})+A_{R}(-\vec{q})\right] / \sqrt{2}, \\
\frac{\partial}{\partial y_{b}(\vec{q})} & =-\sqrt{2} A_{b}^{\dagger}(-\vec{q}), \\
\frac{\partial}{\partial x_{b}(\vec{q})} & =-\sqrt{2} A_{R}^{\dagger}(-\vec{q}) .
\end{aligned}
$$

Hence, (B7a) can be re-expressed as

$$
\begin{aligned}
L_{1}(V, R)= & \sum_{b} \sum_{\vec{q}}\left\{-\Lambda_{b}(\vec{q}) A_{b}^{\dagger}(\vec{q}) A_{b}(\vec{q})\right. \\
& \left.+\sqrt{g_{0, R} \Lambda_{R}(\vec{q})}\left[A_{R}^{\dagger}(\vec{q}) A_{b}(\vec{q})-A_{b}^{\dagger}(\vec{q}) A_{R}(\vec{q})\right]\right\} .
\end{aligned}
$$

Defining the inverse transformations,

$$
\begin{aligned}
& A_{b}^{\dagger}(\vec{q})=-\frac{1}{\sqrt{2}} \frac{\partial}{\partial y_{b}(-\vec{q})}, \\
& A_{b}(\vec{q})=\sqrt{2} y_{b}(-\vec{q})+\frac{1}{\sqrt{2}} \frac{\partial}{\partial y_{b}(\vec{q})},
\end{aligned}
$$

one may show that

$$
\begin{aligned}
{\left[A_{i}(\vec{q}), A_{j}^{\dagger}\left(\vec{q}^{\prime}\right)\right] } & =\delta_{i, j} \delta_{\vec{q}, \vec{q}^{\prime}}, \quad \text { and } \\
A_{b}(\vec{q}) P_{s}(V, R) & =A_{R}(\vec{q}) P_{s}(V, R) \\
& =0 .
\end{aligned}
$$

Equation (B9d) indicates that the $P_{S}$ steady-state probability distribution is formally equivalent to the vacuum state $|0\rangle_{V, R}$ for these operators. Thus, $A_{b}^{\dagger}$ and $A_{R}^{\dagger}$ will generate eigenfunctions in the same fashion as for the quantum mechanical harmonic oscillator [47].

Thus, the expectation values appearing in (A3) will be of the form

$$
\begin{aligned}
\langle f[\chi(\vec{q})]\rangle & =\int A\left(\vec{q}_{1}\right) A^{\dagger}\left(\vec{q}_{2}\right) A\left(\vec{q}_{3}\right) A^{\dagger}\left(\vec{q}_{4}\right) P_{s} d \chi(\vec{q}) \\
& ={ }_{V, R}\left\langle 0\left|A\left(\vec{q}_{1}\right) A^{\dagger}\left(\vec{q}_{2}\right) A\left(\vec{q}_{3}\right) A^{\dagger}\left(\vec{q}_{4}\right)\right| 0\right\rangle_{V, R}
\end{aligned}
$$

and can be routinely calculated. For these calculations it is convenient to write (B8) in terms of the auxiliary operators

$$
b_{1_{+}}(\vec{q}), \quad b_{1_{-}}(\vec{q}), \quad b_{2_{+}}(\vec{q}), \quad b_{2_{-}}(\vec{q}),
$$

where, following Risken [47],

$$
\begin{gathered}
b_{1_{+}}(\vec{q})=\delta^{-\frac{1}{2}}\left[\sqrt{\lambda_{1}(\vec{q})} A_{b}^{\dagger}(\vec{q})-\sqrt{\lambda_{2}(\vec{q})} A_{R}^{\dagger}(\vec{q})\right], \\
b_{1_{-}}(\vec{q})=\delta^{-\frac{1}{2}}\left[\sqrt{\lambda_{1}(\vec{q})} A_{b}(\vec{q})+\sqrt{\lambda_{2}(\vec{q})} A_{R}(\vec{q})\right], \\
b_{2_{+}}(\vec{q})=\delta^{-\frac{1}{2}}\left[-\sqrt{\lambda_{2}(\vec{q})} A_{b}^{\dagger}(\vec{q})+\sqrt{\lambda_{1}(\vec{q})} A_{R}^{\dagger}(\vec{q})\right], \\
b_{2_{-}}(\vec{q})=\delta^{-\frac{1}{2}}\left[\sqrt{\lambda_{2}(\vec{q})} A_{b}(\vec{q})+\sqrt{\lambda_{1}(\vec{q})} A_{R}(\vec{q})\right],
\end{gathered}
$$
with

$$
\begin{aligned}
\lambda_{1}(\vec{q}) & =\left[\Lambda_{b}(\vec{q}) \pm \delta(\vec{q})\right] / 2, \\
\delta(\vec{q}) & =\left[\Lambda_{b}^{2}(\vec{q})-4 \omega_{0}^{2}(\vec{q})\right]^{\frac{1}{2}}, \\
\omega_{0}^{2}(\vec{q}) & =g_{0, R} \Lambda_{R}(\vec{q}) .
\end{aligned}
$$


Invoking these auxiliary operators for (B8) gives

$$
\begin{aligned}
L_{1}(V, R)= & \sum_{\vec{q}}\left[\lambda_{1}(\vec{q}) b_{1_{+}}(\vec{q}) b_{1_{-}}(\vec{q})\right. \\
& \left.-\lambda_{2}(\vec{q}) b_{2_{+}}(\vec{q}) b_{2_{-}}(\vec{q})\right] .
\end{aligned}
$$

$L_{1}$ acts on the eigenfunction $\psi_{n_{1}, n_{2}}^{(V, R)}$,

$$
L_{1}(V, R) \psi_{n_{1}, n_{2}}^{(V, R)}(\vec{q})=-\lambda_{n_{1}, n_{2}}(\vec{q}) \psi_{n_{1}, n_{2}}^{(V, R)}(\vec{q})
$$

where

$$
\lambda_{n_{1}, n_{2}}(\vec{q})=n_{1} \lambda_{1}(\vec{q})+n_{2} \lambda_{2}(\vec{q})
$$

and

$$
\psi_{n_{1}, n_{2}}^{(V, R)}(\vec{q})=\left(n_{1} ! n_{2} !\right)^{-\frac{1}{2}}\left[b_{1_{+}}(\vec{q})\right]^{n_{1}}\left[b_{2_{+}}(\vec{q})\right]^{n_{2}}|0\rangle_{V, R} .
$$

Following a similar procedure, we obtain expressions for $L_{1}(Z, \Phi)$ and $L_{1}(\Pi, \phi)$,

$$
\begin{aligned}
L_{1}(Z, \Phi)= & \sum_{a, b} \sum_{\vec{q}}\left\{-\bar{a}_{2} A_{z}^{\dagger}(\vec{q}) A_{z}(\vec{q})\right. \\
& \left.+\sqrt{\bar{a}_{3}}\left[A_{p}^{\dagger}(\vec{q}) A_{z}(\vec{q})-A_{z}^{\dagger}(\vec{q}) A_{p}(\vec{q})\right]\right\}, \\
L_{1}(\Pi, \phi)= & \sum_{a, b} \sum_{\vec{q}}\left\{-2 v \Lambda_{a b} A_{r}^{\dagger}(\vec{q}) A_{r}(\vec{q})\right. \\
& \left.+\sqrt{\bar{a}_{4}}\left[A_{s}^{\dagger}(\vec{q}) A_{r}(\vec{q})-A_{r}^{\dagger}(\vec{q}) A_{s}(\vec{q})\right]\right\},
\end{aligned}
$$

where $\left[A_{z}^{\dagger}(\vec{q}), A_{p}^{\dagger}(\vec{q})\right]$ are creation operators acting on the $|0\rangle_{Z, \Phi}$ vacuum state, while $\left[A_{r}^{\dagger}(\vec{q}), A_{s}^{\dagger}(\vec{q})\right]$ act on the $|0\rangle_{\Pi, \phi}$ vacuum state. These vacuum or ground states are defined by their respective stationary probability distributions,

$$
\begin{aligned}
P_{S}(Z, \Phi)= & \mathscr{N} \exp \left[-\frac{2 \ell^{2}}{\Gamma_{Z}} \bar{a}_{2} Z_{a b}(\vec{q}) Z_{a b}(-\vec{q})\right] \\
& \times \exp \left[-\frac{2 \ell^{2}}{\Gamma_{Z}} \bar{a}_{2} \bar{a}_{3} \Phi_{a b}(\vec{q}) \Phi_{a b}(-\vec{q})\right] \equiv|0\rangle_{Z, \Phi}, \\
P_{S}(\Pi, \phi)= & \mathscr{N} \exp \left[-\frac{2 \ell^{2}}{\Gamma_{\Pi}} 2 v \Lambda_{a b} \Pi_{a b}(\vec{q}) \Pi_{a b}(-\vec{q})\right] \\
& \times \exp \left[-\frac{2 \ell^{2}}{\Gamma_{\Pi}} 2 \bar{a}_{4} v \Lambda_{a b} \phi_{a b}(\vec{q}) \phi_{a b}(-\vec{q})\right] \equiv|0\rangle_{\Pi, \phi} .
\end{aligned}
$$

In summary, the stationary solution of

$$
L_{1}=L_{1}(V, R)+L_{1}(Z, \Phi)+L_{1}(\Pi, \phi)
$$

is

$$
L_{1} P_{s}=0
$$

where

$$
P_{s}=P_{s}(V, R) P_{s}(Z, \Phi) P_{s}(\Pi, \phi)
$$

is the Fock-space ground state for the complete cortical model,

$$
|0\rangle=|0\rangle_{V, R}|0\rangle_{Z, \Phi}|0\rangle_{\Pi, \phi} .
$$

Finally, we note that the operators defined in (B15) and (B16) may be recast into forms corresponding to those given in (B12)-(B14).

\section{APPENDIX C: EVALUATION OF EXPECTATION$$
\left\langle\mathscr{L}_{3} L_{1}^{-1} L_{2}\right\rangle
$$

Complete evaluation of the RHS of (A2) involves manipulation of a large number of terms, since each of $L_{2}, L_{3}$ must be reexpressed in terms of creation and annihilation operators. To indicate the essential method to the reader, we focus here on the evaluation of a single exemplar, namely $\left\langle\mathscr{L}_{3} L_{1}^{-1} L_{2}\left(A_{b}^{\dagger}\right)\right\rangle$.

The rightmost component of the expectation is written as the sum of the five subcomponents $L_{2, j}$

$$
\begin{aligned}
L_{2}\left(A_{b}^{\dagger}\right)= & L_{2,1}\left(\vec{q}, \vec{q}_{1}, \vec{q}_{2}\right) A_{b}^{\dagger}(-\vec{q}) A_{b}\left(-\vec{q}_{1}\right) A_{b}^{\dagger}\left(\vec{q}_{2}\right) \delta_{\vec{q}, \vec{q}_{1}+\vec{q}_{2}} \\
& +L_{2,2}(\vec{q}) A_{b}^{\dagger}(-\vec{q}) \delta_{q, \vec{Q}_{1}+\vec{Q}_{2}} \\
& +L_{2,3}\left(\vec{q}, \vec{q}_{1}, \vec{q}_{2}\right) A_{b}^{\dagger}(-\vec{q}) A_{b}\left(-\vec{q}_{1}\right) A_{b}^{\dagger}\left(\vec{q}_{2}\right) \delta_{\vec{q}, \vec{q}_{1}+\vec{q}_{2}+\vec{Q}_{3}} \\
& +L_{2,4}(\vec{q}) A_{b}^{\dagger}(-\vec{q}) \delta_{\vec{q}, \vec{Q}_{1}+\vec{Q}_{2}+\vec{Q}_{3}} \\
& +L_{2,5}(\vec{q}) A_{b}^{\dagger}(-\vec{q}) \delta_{\vec{q}, \vec{Q}_{1}+\vec{Q}_{2} .}
\end{aligned}
$$

Noting that when $L_{2,1}$ and $L_{2,3}$ act on the vacuum, they produce

$$
\delta_{-\vec{q}_{1}, \vec{q}_{2}} \delta_{\vec{q}, \vec{q}_{1}+\vec{q}_{2}}=\delta_{\vec{q}, 0}=0
$$

so we need only consider

$$
\begin{aligned}
& L_{2}\left(A_{b}^{\dagger}\right)
\end{aligned}
$$

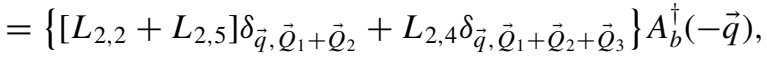

where

$$
\begin{aligned}
L_{2,2} & =\frac{a_{3, b}}{2 n+1} C_{b}(\vec{q}) V_{b}\left(\vec{Q}_{1}\right) V_{b}\left(\vec{Q}_{2}\right), \\
L_{2,4} & =\frac{a_{2, b}}{(2 n+1)^{2}} C_{b}(\vec{q}) V_{b}\left(\vec{Q}_{1}\right) V_{b}\left(\vec{Q}_{2}\right) V_{b}\left(\vec{Q}_{3}\right), \\
L_{2,5} & =\frac{g_{R, b}}{2 n+1} C_{b}(\vec{q}) R_{b}\left(\vec{Q}_{1}\right) V_{b}\left(\vec{Q}_{2}\right),
\end{aligned}
$$

with

$$
C_{b}(\vec{q})=-\ell \sqrt{\frac{2 \Lambda_{b}(\vec{q})}{\Gamma_{b}}} .
$$

Using (B10), we find

$$
L_{1}^{-1} A_{b}^{\dagger}|0\rangle=-\frac{A_{R}^{\dagger}(\vec{q})}{\omega_{0}}|0\rangle,
$$

with $\omega_{0}^{2}=g_{0, R} \Lambda_{R}(\vec{q})$. Thus, the only term from $\mathscr{L}_{3}$ to contribute to the $\langle\cdot\rangle$ expectation is $\mathscr{L}_{3}\left(A_{R}\right)$, which, omitting explicit summations, is

$$
\mathscr{L}_{3}\left(A_{R}\right)=\mathscr{L}_{3,1}\left(\vec{Q}, \vec{Q}_{2}, \vec{q}_{1}\right) A_{R}\left(-\vec{q}_{1}\right) \delta_{\vec{Q}, \vec{q}_{1}+\vec{Q}_{2}},
$$

where

$$
\mathscr{L}_{3,1}\left(\vec{Q}, \vec{Q}_{2}, \vec{q}_{1}\right)=\frac{g_{R, b}}{(2 n+1) \ell} \sqrt{\frac{\Gamma_{b} \Lambda_{R}\left(\vec{q}_{1}\right)}{2 g_{0, R} \Lambda_{b}\left(\vec{q}_{1}\right)}} \frac{\partial V_{b}\left(\vec{Q}_{2}\right)}{\partial V_{b}(\vec{Q})} .
$$


Thus,

$$
\begin{aligned}
& \left\langle\mathscr{L}_{3} L_{1}^{-1} L_{2}\left(A_{b}^{\dagger}\right)\right\rangle \\
& =-\sum_{[\vec{Q}, \vec{q}, b]} \mathscr{L}_{3,1}\left(\vec{Q}, \vec{Q}_{2}, \vec{q}_{1}\right) \\
& \times \frac{\left\langle A_{R}\left(-\vec{q}_{1}\right) A_{R}^{\dagger}\left(\vec{q}^{\prime}\right)\right\rangle}{\omega_{0}\left(\vec{q}^{\prime}\right)}\left\{\left[L_{2,2}\left(\vec{q}^{\prime}\right)+L_{2,5}\left(\vec{q}^{\prime}\right)\right]\right. \\
& \left.\times \delta_{\vec{q}^{\prime}, \vec{Q}_{1}^{\prime}+\vec{Q}_{2}^{\prime}}+L_{2,4}\left(\vec{q}^{\prime}\right) \delta_{\vec{q}^{\prime}, \vec{Q}_{1}^{\prime}+\vec{Q}_{2}^{\prime}+\vec{Q}_{3}^{\prime}}\right\} \delta_{\vec{Q}, \vec{q}_{1}+\vec{Q}_{2}} .
\end{aligned}
$$

Summing over $\vec{q}$ gives

$$
\begin{aligned}
& \langle\cdot\rangle=\sum_{[\vec{Q}, b]} \frac{g_{R, b}}{g_{0, R}}\left\{\frac{1}{(2 n+1)^{2}} \frac{\partial}{\partial V_{b}(\vec{Q})} V_{b}\left(\vec{Q}_{2}\right)\right. \\
& \times\left[a_{3, b} V_{b}\left(\vec{Q}_{1}^{\prime}\right) V_{b}\left(\vec{Q}_{2}^{\prime}\right)+g_{R, b} R_{b}\left(\vec{Q}_{1}^{\prime}\right) V_{b}\left(\vec{Q}_{2}^{\prime}\right)\right] \delta_{\vec{Q}, \vec{Q}_{1}^{\prime}+\vec{Q}_{2}^{\prime}+\vec{Q}_{2}} \\
& +\frac{a_{2, b}}{(2 n+1)^{3}} \frac{\partial}{\partial V_{b}(\vec{Q})} V_{b}\left(\vec{Q}_{2}\right) V_{b}\left(\vec{Q}_{1}^{\prime}\right) V_{b}\left(\vec{Q}_{2}^{\prime}\right) V_{b}\left(\vec{Q}_{3}^{\prime}\right)
\end{aligned}
$$

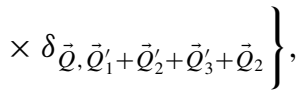

where the square-bracketed indexing notation $[\vec{Q}, b]$ signals that all possible sums (over $\vec{Q}, \vec{Q}_{1}^{\prime}, e$, $i$, etc.) are to be considered.

Fourier inversion from wave-number space back to physical space cannot be done using the generic methods outlined in Appendix D; instead, special handling is required because of the implicit restrictions on the $\vec{Q}$ summations.

We demonstrate with the first term of (C3),

$$
\begin{aligned}
& \sum_{[\vec{Q}, b]} \frac{a_{3, b} g_{R, b}}{g_{0, R}} \frac{1}{(2 n+1)^{2}} \frac{\partial}{\partial V_{b}(\vec{Q})} V_{b}\left(\vec{Q}_{2}\right) V_{b}\left(\vec{Q}_{1}^{\prime}\right) V_{b}\left(\vec{Q}_{2}^{\prime}\right) \\
& \quad \times \delta_{\vec{Q}, \vec{Q}_{1}^{\prime}+\vec{Q}_{2}^{\prime}+\vec{Q}_{2} .}
\end{aligned}
$$

From the $\delta$ functions embedded in (C2) we know that

$$
\vec{q}^{\prime}=\vec{Q}_{1}^{\prime}+\vec{Q}_{2}^{\prime}, \quad \vec{q}_{1}=\vec{Q}-\vec{Q}_{2} .
$$

After applying Fourier mappings (62c) and (62e) and summing over $\vec{Q}$, we find that expression (C4) becomes

$$
\begin{aligned}
& \sum_{[\vec{Q}, \vec{J}, b]} \frac{a_{3, b} g_{R, b} / g_{0, R}}{(2 n+1)^{2}(2 N+1)^{4}} \frac{\partial}{\partial V_{b, \vec{J}}}\left[V_{b, \vec{J}_{2}} V_{b, \vec{J}_{1}^{\prime}} V_{b, \vec{J}_{2}^{\prime}}\right] \\
& \quad \times e^{i\left(\vec{J}-\vec{J}_{2}\right) \cdot \vec{Q}_{2} L} e^{i\left(\vec{J}-\vec{J}_{1}^{\prime}\right) \cdot \vec{Q}_{1}^{\prime} L} e^{i\left(\vec{J}-\vec{J}_{2}^{\prime}\right) \cdot \vec{Q}_{2}^{\prime} L} .
\end{aligned}
$$

We evaluate the sums over $\vec{Q}$ as

$$
\begin{aligned}
\sum_{\vec{Q}_{2}} e^{i\left(\vec{J}-\vec{J}_{2}\right) \cdot \vec{Q}_{2} L} & =\left[\frac{(2 n+1) \ell}{2 \pi}\right]^{2} \int e^{i\left(\vec{J}-\vec{J}_{2}\right) \cdot \vec{Q}_{2} L} d \vec{Q}_{2} \\
& \approx \delta_{\vec{J}, \vec{J}_{2}}\left[\frac{(2 n+1) \ell}{2 \pi}\right]^{2} \int d \vec{Q}_{2} \\
& =S_{1} \cdot(2 N+1)^{2} \delta_{\vec{J}, \vec{J}_{2}},
\end{aligned}
$$

where $S_{1} \approx 0.4375$ is the dilution factor on $\int d \vec{Q}_{2}$ arising from the second wave-number restriction $\vec{q}_{1}=\vec{Q}-\vec{Q}_{2}$ of (C5), namely that the difference of two vectors in $\vec{Q}$ space must lie in $\vec{q}$ space, i.e., must lie outside the $\vec{Q}$-space square.
In view of the first restriction $\vec{q}^{\prime}=\vec{Q}_{1}^{\prime}+\vec{Q}_{2}^{\prime}$ of (C5), the two remaining $\vec{Q}_{1}^{\prime}$ and $\vec{Q}_{2}^{\prime}$ summations are paired, giving

$$
\begin{aligned}
& \sum_{\vec{Q}_{1}^{\prime}, \vec{Q}_{2}^{\prime}} e^{i\left(\vec{J}-\vec{J}_{1}^{\prime}\right) \cdot \vec{Q}_{1}^{\prime} L} e^{i\left(\vec{J}-\vec{J}_{2}^{\prime}\right) \cdot \vec{Q}_{2}^{\prime} L} \\
& \quad \approx \delta_{\vec{J}, \vec{J}_{1}^{\prime}} \delta_{\vec{J}, \vec{J}_{2}^{\prime}}\left[\frac{(2 n+1) \ell}{2 \pi}\right]^{4} \iint d \vec{Q}_{1}^{\prime} d \vec{Q}_{2}^{\prime} \\
& \quad=S_{1}(2 N+1)^{4} \delta_{\vec{J}, \vec{J}_{1}^{\prime}} \delta_{\vec{J}, \vec{J}_{2}^{\prime}} ;
\end{aligned}
$$

thus, expression (C4) becomes

$$
\sum_{[\vec{J}, b]} \frac{a_{3, b} g_{R, b}}{g_{0, R}} \frac{S_{1}^{2}}{B^{2}} \frac{\partial}{\partial V_{b, \vec{J}}} V_{b, \vec{J}}^{3} \cdot
$$

Using similar methods, we complete the Fourier inversion of (C3) to coarse-grained $\vec{J}$ space so that $\left\langle\mathscr{L}_{3} L_{1}^{-1} L_{2}\left(A_{b}^{\dagger}\right)\right\rangle$ transforms to

$$
\begin{aligned}
& \sum_{[\vec{J}, b]} \frac{\partial}{\partial \widetilde{V}_{b, \vec{J}}}\left\{\frac{a_{3, b} g_{R, b} S_{1}^{2}}{g_{0, R}} \widetilde{V}_{b, \vec{J}}^{3}+\frac{g_{R, b}^{2} S_{1}^{2}}{g_{0, R}} \widetilde{V}_{b, \vec{J}}^{2} \widetilde{R}_{b, \vec{J}}\right. \\
& \left.\quad+\frac{a_{2, b} g_{R, b} S_{1} S_{2}}{g_{0, R}} \widetilde{V}_{b, \vec{J}}^{4}\right\},
\end{aligned}
$$

where $S_{2} \approx 0.5555$ is the scaling factor arising from an integration that requires a triple-vector restriction $\vec{q}=\vec{Q}_{1}+$ $\vec{Q}_{2}+\vec{Q}_{3}$.

\section{APPENDIX D: INVERSION OF GENERIC DRIFT AND DIFFUSION TERMS}

We now describe the standard techniques used to invert so-called "generic" drift and diffusion terms, that is, terms that do not have any $\delta$-function restrictions in $\vec{Q}$ space.

\section{Inversion of drift terms}

In $\vec{Q}$ space, generic drift terms take the form

$$
\sum_{[\vec{Q}]} \frac{\partial}{\partial \chi(\vec{Q})} \prod_{j=1}^{M} \chi^{\prime}\left(\vec{Q}_{j}\right) \delta_{\left[\vec{Q}, \sum_{j=1}^{M} \vec{Q}_{j}\right]},
$$

with $\left\{\chi, \chi^{\prime}\right\} \in\{V, R, Z, \Phi, \Pi, \phi\}$. Using the inverse transformations (62c) and (62e), drift expression (D1) becomes

$$
\begin{aligned}
\sum_{[\vec{J}, \vec{Q}]} \frac{\partial}{\partial \chi_{\vec{J}}} \widetilde{f}_{\vec{J}}(\vec{Q}) \prod_{j=1}^{M}\left\{\tilde{f}_{\vec{J}_{j}}^{*}\left(\vec{Q}_{j}\right) \chi_{\vec{J}_{j}}^{\prime}\right\} \delta_{\left[\vec{Q}, \sum_{j=1}^{M} \vec{Q}_{j}\right]} \\
=\frac{1}{(2 N+1)^{M+1}} \sum_{[\vec{J}, \vec{Q}]} \frac{\partial}{\partial \chi_{\vec{J}}} e^{i \vec{J} \cdot \vec{Q} L} e^{-i \sum_{j} \vec{J}_{j} \cdot \vec{Q}_{j} L} \\
\quad \times \prod_{j=1}^{M} \chi_{\vec{J}_{j}}^{\prime} \delta_{\left[\vec{Q}, \sum_{j=1}^{M} \vec{Q}_{j}\right]}
\end{aligned}
$$

We invoke the $\delta$-function property and note that

$$
\sum_{\vec{Q}_{j}} e^{i\left(\vec{J}-\vec{J}_{j}\right) \cdot \vec{Q}_{j} L}=(2 N+1)^{2} \delta_{\vec{J}, \vec{J}_{j}},
$$


so that (D2) becomes

$$
\begin{aligned}
& \frac{(2 N+1)^{2 M}}{(2 N+1)^{M+1}} \sum_{\vec{J}} \frac{\partial}{\partial \chi_{\vec{J}}}\left[\chi_{\vec{J}}^{\prime}\right]^{M} \\
& =(2 N+1)^{M-1} \sum_{\vec{J}} \frac{\partial}{\partial \tilde{\chi}_{\vec{J}}}\left[\tilde{\chi}_{\vec{J}}^{\prime}\right]^{M},
\end{aligned}
$$

where we have mapped $\left\{\chi, \chi^{\prime}\right\} \rightarrow\left\{\tilde{\chi}, \tilde{\chi}^{\prime}\right\}$ using the coarsegrain scaling definition of Eq. (61).

\section{Inversion of diffusion terms}

In $\vec{Q}$ space, the generic diffusion term takes the form

$$
\begin{aligned}
& \sum_{[\vec{Q}]} \frac{\partial}{\partial \chi(\vec{Q})}\left\{\prod_{j=1}^{M} \chi^{\prime}\left(\vec{Q}_{j}\right)\right\} \frac{\partial}{\partial W\left(\vec{Q}^{\prime}\right)}\left\{\prod_{j^{\prime}=1}^{M^{\prime}} W_{j^{\prime}}^{\prime}\left(\vec{Q}_{j}^{\prime}\right)\right\} \\
& \quad \times \delta_{\left[\vec{Q}, \sum_{j=1}^{M} \vec{Q}_{j}+\sum_{j^{\prime}=1}^{M^{\prime}} \vec{Q}_{j}^{\prime}-\vec{Q}^{\prime}\right]},
\end{aligned}
$$

with $\left\{\chi, \chi^{\prime}, W, W^{\prime}\right\} \in\{V, R, Z, \Pi\}$. Following the procedures outlined above for the (D1) drift, this diffusion maps to coarsegrained $\vec{J}$ space as

$$
(2 N+1)^{\left(M+M^{\prime}\right)} B^{\left(M+M^{\prime}-2\right)} \sum_{\vec{J}} \frac{\partial}{\partial \widetilde{\chi}_{\vec{J}}}\left[\widetilde{\chi}_{\vec{J}}^{\prime}\right]^{M} \frac{\partial}{\partial \widetilde{W}_{\vec{J}}}\left[\widetilde{W}_{\vec{J}}^{\prime}\right]^{M^{\prime}}
$$

where $B=(2 n+1) /(2 N+1)$ is the blocking ratio linking the fine and coarse spatial scales.

\section{APPENDIX E: DEFINITIONS FOR WAVE-NUMBER INTEGRALS}

This Appendix itemizes the $\bar{c}_{i}$ wave-number integrals referenced in the $d_{i}$ drift coefficients of Eq. (59) and $g_{i}$ diffusion coefficients of Eq. (60). The integrals are evaluated numerically, taking into account any restrictions on the wavenumber ranges as flagged by labels R0, R1 and R2; these restrictions are defined below [we list the SI units for each integral to aid checking of model equations for dimensional consistency, thus reducing occurrences of typographical and other errors]:

$$
\begin{array}{llr}
\bar{c}_{0}=\int \frac{d \vec{q}}{\Lambda_{b}(\vec{q})} & {\left[\mathrm{m}^{-2} \mathrm{~s}\right],} \\
\bar{c}_{1}=-\int \frac{d \vec{q}}{2\left[\Lambda_{b}(\vec{q})\right]^{2}} & {\left[\mathrm{~m}^{-2} \mathrm{~s}^{2}\right],} \\
\bar{c}_{2}=-\int \frac{d \vec{q}}{2\left[\Lambda_{b}(\vec{q})\right]^{3}} & {\left[\mathrm{~m}^{-2} \mathrm{~s}^{3}\right],} \\
\bar{c}_{3}=\iint \frac{g\left(\vec{q}_{1}, \vec{q}_{2}\right) d \vec{q}_{1} d \vec{q}_{2}}{\Lambda_{b}\left(\vec{q}_{1}\right) \Lambda_{b}\left(\vec{q}_{2}\right)} \mathrm{R} 0 & {\left[\mathrm{~m}^{-4} \mathrm{~s}^{3}\right],} \\
\bar{c}_{4}=\iint \frac{d \vec{Q}_{1} d \vec{Q}_{2}}{\Lambda_{R}\left(\vec{Q}_{1}+\vec{Q}_{2}\right)} \mathrm{R} 1 & {\left[\mathrm{~m}^{-4} \mathrm{Vs}\right],} \\
\bar{c}_{5}=\iint \frac{\Lambda_{b}\left(\vec{Q}_{1}+\vec{Q}_{2}\right) d \vec{Q}_{1} d \vec{Q}_{2}}{\Lambda_{R}\left(\vec{Q}_{1}+\vec{Q}_{2}\right)} \mathrm{R} 1 & {\left[\mathrm{~m}^{-4} \mathrm{~V}\right],} \\
\bar{c}_{6 a}=\iint \frac{h\left(\vec{q}_{1}, \vec{q}_{2}\right) \sqrt{\Lambda_{R}\left(\vec{q}_{1}+\vec{q}_{2}\right)} d \vec{q}_{1} d \vec{q}_{2}}{\Lambda_{b}\left(\vec{q}_{1}\right) \Lambda_{b}\left(\vec{q}_{1}+\vec{q}_{2}\right)} & \mathrm{R} 0 & {\left[\frac{\mathrm{s}^{5 / 2}}{\left.\mathrm{~m}^{4} \mathrm{~V}^{1 / 2}\right]},\right.}
\end{array}
$$

$$
\begin{aligned}
& \bar{c}_{6 b}=\iint \frac{h\left(\vec{q}_{1}, \vec{q}_{2}\right) d \vec{q}_{1} d \vec{q}_{2}}{\Lambda_{b}\left(\vec{q}_{1}\right) \Lambda_{b}\left(\vec{q}_{2}\right) \sqrt{\Lambda_{R}\left(\vec{q}_{1}+\vec{q}_{2}\right)}} \mathrm{R} 0 \quad\left[\frac{\mathrm{V}^{1 / 2} \mathrm{~s}^{7 / 2}}{\mathrm{~m}^{4}}\right], \\
& \bar{c}_{7}=\bar{c}_{1} \\
& \bar{c}_{8}=-\int \frac{d \vec{q}}{2 \Lambda_{b}(\vec{q}) \Lambda_{R}(\vec{q})} \\
& \bar{c}_{9}=\frac{\bar{c}_{0}}{\sqrt{g_{0, R}}} \\
& \bar{c}_{10}=\frac{1}{\sqrt{g_{0, R}}} \int \frac{d \vec{q}}{\Lambda_{R}(\vec{q})} \\
& \bar{c}_{11}=\bar{c}_{2} \\
& \bar{c}_{12}=\iint \frac{g\left(\vec{q}_{1}, \vec{q}_{2}\right) d \vec{q}_{1} d \vec{q}_{2}}{\Lambda_{b}\left(\vec{q}_{1}\right) \Lambda_{b}\left(\vec{q}_{2}\right) \Lambda_{b}\left(\vec{q}_{1}+\vec{q}_{2}\right)} \mathrm{R} 0 \quad\left[\mathrm{~m}^{-4} \mathrm{~s}^{4}\right], \\
& \bar{c}_{15}=\iint \frac{d \vec{Q}_{1} d \vec{Q}_{2}}{\Lambda_{b}\left(\vec{Q}_{1}+\vec{Q}_{2}\right)} \mathrm{R} 1 \quad\left[\mathrm{~m}^{-4} \mathrm{~s}\right] \text {, } \\
& \bar{c}_{16}=\iiint \frac{d \vec{Q}_{1} d \vec{Q}_{2} d \vec{Q}_{3}}{\Lambda_{b}\left(\vec{Q}_{1}+\vec{Q}_{2}+\vec{Q}_{3}\right)} \mathrm{R} 2 \quad\left[\mathrm{~m}^{-6} \mathrm{~s}\right] \text {, } \\
& {\left[\mathrm{m}^{-2} \mathrm{~s}^{2}\right],} \\
& {\left[\mathrm{m}^{-2} \mathrm{Vs}^{2}\right],} \\
& {\left[\mathrm{m}^{-2} \mathrm{~V}^{-1 / 2} \mathrm{~s}^{3 / 2}\right],} \\
& {\left[\mathrm{m}^{-2} \mathrm{~V}^{1 / 2} \mathrm{~s}^{3 / 2}\right],} \\
& {\left[\mathrm{m}^{-2} \mathrm{~s}^{3}\right],}
\end{aligned}
$$

where the wave-number restrictions are stipulated as

$$
\begin{array}{lll}
\mathrm{R} 0: & \vec{q}_{1}+\vec{q}_{2}=\vec{q} & \left(\bar{c}_{3}, \bar{c}_{6 a}, \bar{c}_{6 b}, \bar{c}_{12}\right), \\
\mathrm{R} 1: & \vec{Q}_{1}+\vec{Q}_{2}=\vec{q} & \left(\bar{c}_{4}, \bar{c}_{5}, \bar{c}_{15}\right), \\
\mathrm{R} 2: & \vec{Q}_{1}+\vec{Q}_{2}+\vec{Q}_{3}=\vec{q} & \left(\bar{c}_{16}\right),
\end{array}
$$

and where the $\Lambda_{b}$ and $\Lambda_{R}$ definitions are given in (B2b) and (B2c), respectively. The two-argument functions $g(\cdot)$ (appearing in the $\bar{c}_{3}$ and $\bar{c}_{12}$ integrals) and $h(\cdot)$ (in $\bar{c}_{6 a}$ and $\left.\bar{c}_{6 b}\right)$ depend on the wave-number sum $\vec{q}_{1}+\vec{q}_{2}=\vec{q}_{3}$ and are defined as

$$
\begin{aligned}
g\left(\vec{q}_{1}, \vec{q}_{2}, \vec{q}_{3}\right)= & \Delta_{1}^{2} \\
& \times\left\{-\frac{L_{11} L_{12} L_{13}}{L_{11}+L_{12}+L_{13}}+\frac{L_{11} L_{12} L_{23}}{L_{11}+L_{12}+L_{23}}\right. \\
& +\frac{L_{11} L_{22} L_{13}}{L_{11}+L_{22}+L_{13}}-\frac{L_{11} L_{22} L_{23}}{L_{11}+L_{22}+L_{23}} \\
& +\frac{L_{21} L_{12} L_{13}}{L_{21}+L_{12}+L_{13}}-\frac{L_{21} L_{12} L_{23}}{L_{21}+L_{12}+L_{23}} \\
& \left.-\frac{L_{21} L_{22} L_{13}}{L_{21}+L_{22}+L_{13}}+\frac{L_{21} L_{22} L_{23}}{L_{21}+L_{22}+L_{23}}\right\}
\end{aligned}
$$

and

$$
\begin{aligned}
h\left(\vec{q}_{1}, \vec{q}_{2}, \vec{q}_{3}\right)= & \Delta_{1}^{2} \sqrt{L_{11} L_{21}} \\
& \times\left\{-\frac{L_{12} L_{13}}{L_{11}+L_{12}+L_{13}}+\frac{L_{12} L_{23}}{L_{11}+L_{12}+L_{23}}\right. \\
& +\frac{L_{22} L_{13}}{L_{11}+L_{22}+L_{13}}-\frac{L_{22} L_{23}}{L_{11}+L_{22}+L_{23}} \\
& +\frac{L_{12} L_{13}}{L_{21}+L_{12}+L_{13}}-\frac{L_{12} L_{23}}{L_{21}+L_{12}+L_{23}} \\
& \left.-\frac{L_{22} L_{13}}{L_{21}+L_{22}+L_{13}}+\frac{L_{22} L_{23}}{L_{21}+L_{22}+L_{23}}\right\},
\end{aligned}
$$


where the $L_{m n}$ represent

$$
L_{m n}=\lambda_{m}\left(\vec{q}_{n}\right),
$$

with $\lambda_{m} \in\left\{\lambda_{1}, \lambda_{2}\right\}$ and $\vec{q}_{n} \in\left\{\vec{q}_{1}, \vec{q}_{2}, \vec{q}_{3}\right\}$, and where

$$
\Delta_{1}^{2}=\frac{1}{\delta\left(\vec{q}_{1}\right) \delta\left(\vec{q}_{2}\right) \delta\left(\vec{q}_{3}\right)} .
$$

We recall that $\lambda_{m}(\vec{q})$ and $\delta(\vec{q})$ have been defined in (B11a) and (B11b) of Appendix B.

\section{APPENDIX F: GAP-JUNCTION COUPLING AND $L_{1}$ DOMINANCE}

Convergence of our perturbative expansion requires sufficient GJ diffusion $D_{b}>0$ such that the Laplacian terms of $L_{1}$ dominate the denominator of Eq. (50d). We estimate the minimum coupling strength required for convergence by comparing the relative sizes of the Laplacian and $\mathrm{Na}^{+}$-flux terms in the equation of motion (20) for $V_{b}$. The largest possible wave number supported by the micro-scale cortical grid (and the first to be eliminated in the reblocking) is $\vec{q}^{\max }$ whose $x$ and $y$ components are $q_{x}=q_{y}=\pi / \ell$ (see Fig. 4). We impose a standing wave on the $2 \mathrm{D}$ cortical grid,

$$
V(\vec{r})=V_{0}+V_{1} e^{i \vec{q} \cdot \vec{r}},
$$

with $V_{0}=-50 \mathrm{mV}$ and $V_{1}=8 \mathrm{mV}$ which might correspond to the $\sim 16-\mathrm{mV}$ peak-to-peak variation between the "up" and "down" states of slow-wave sleep. For this voltage range, maximum $\mathrm{Na}^{+}$flux occurs at $V=-42 \mathrm{mV}$,

$$
-g(V)\left(V-E_{\mathrm{K}}\right) \approx 350 \mathrm{~V} / \mathrm{s},
$$

while the Laplacian term generates a flux

$$
-D_{b} \nabla^{2} V(\vec{r})=\left(q_{x}^{2}+q_{y}^{2}\right) D_{b} V_{1} e^{i \vec{q} \cdot \vec{r}}
$$

of amplitude

$$
2(\pi / \ell)^{2} D_{b} V_{1} \approx 1.04 \times 10^{6} \mathrm{~V} / \mathrm{m}
$$

Here $\ell=10 \mu \mathrm{m}$ and $D_{b}=6.60 \times 10^{-4} \mathrm{~m}^{2} / \mathrm{s}$ is the (large) value for GJ diffusivity used earlier in the exemplar of Sec. III. The Laplacian flux is about 3000 times larger than the maximal $\mathrm{Na}^{+}$flux, so we can safely lower $D_{b}$ by three orders of magnitude to establish a minimum "safe" level for the GJ coupling coefficient between pairs of electrically connected neurons. Working with the Ref. [41] analysis of the Fukuda et al. [48] measurements of interneuronal GJ abundances,

$$
10^{-3} D_{b}=\frac{a N}{4 T} \frac{R_{m}}{R_{g}} \Rightarrow \frac{R_{m}}{R_{g}}=0.011,
$$

where $a=0.16 \mathrm{~mm}^{2}$ is the area of a diffusive "Fukuda square," $N=60$ is the average number of GJ connections per neuron (divided by four to give the number of connections per side), and $T=40 \mathrm{~ms}$ is the neuron time constant. $R_{g}$ is the interneuron gap-coupling resistance, and $R_{m}$ is the membrane (or input) resistance of the target cell; the ratio $R_{m} / R_{g}$ determines the voltage coupling coefficient $c$ (see Fig. 2 of Ref. [79]),

$$
c=\frac{R_{m}}{R_{g}+R_{m}}=\frac{R_{m} / R_{g}}{1+R_{m} / R_{g}}=0.0109 \approx 1 \% .
$$

Wang et al. [53] report coupling coefficients for inhibitoryinhibitory pairings ranging from $6 \%$ to $17 \%$, well above the $1 \%$ minimum we require for convergence of our perturbative theory for spatial reblocking.

Because GJ connections between pairs of excitatory neurons are very sparse, we expect that the dominant terms of $L_{1}$ will be determined exclusively by the inhibitory diffusive contribution alone. The practical outcome of this will be that the sum over index $b$ in Eq. (B8) will collapse to the single instance $b=i$. Note that the blocking procedure will still be valid, producing the coarse-grained Fokker-Planck (FP) equation (57) in which the first term contains the spatially rescaled FP equation. However, the additional correction terms defined in Eqs (59) for drift and (60) for diffusion will now only contain inhibitory contributions, so once again the sum over $b$ will collapse to $b=i$.

In effect, the presence of ubiquitous inhibitory GJ connectivity supports spatial coarse-graining across both populations.
[1] A. L. Hodgkin and A. F. Huxley, A quantitative description of membrane current and its application to conduction and excitation in nerve, J. Physiol. 117, 500 (1952).

[2] R. FitzHugh, Impulses and physiological states in models of nerve membrane, Biophys. J. 1, 445 (1961).

[3] J. S. Nagumo, S. Arimoto, and S. Yoshizawa, An active pulse transmission line simulating a nerve axon, Proc. IRE 50, 2061 (1962).

[4] H. R. Wilson, Spikes, Decisions and Actions: The Dynamical Foundations of Neuroscience (Oxford University Press, New York, 1999).

[5] H. R. Wilson, Simplified dynamics of human and mammalian neocortical neurons, J. Theor. Biol. 200, 375 (1999).

[6] R. F. Fox and Y. Lu, Emergent collective behavior in large numbers of globally coupled independently stochastic ion channels, Phys. Rev. E 49, 3421 (1994).
[7] Elad Schneidman, Barry Freedman, and Idan Segev, Ion channel stochasticity may be critical in determining the reliability and precision of spike timing, Neural Comput. 10, 1679 (1998).

[8] D. A. Steyn-Ross, M. L. Steyn-Ross, M. T. Wilson, and J. W. Sleigh, White-noise susceptibility and critical slowing in neurons near spiking threshold, Phys. Rev. E 74, 051920 (2006).

[9] Joshua H. Goldwyn, Nikita S. Imennov, Michael Famulare, and Eric Shea-Brown, Stochastic differential equation models for ion channel noise in Hodgkin-Huxley neurons, Phys. Rev. E 83, 041908 (2011)

[10] A. Destexhe and M. Rudolph-Lilith, Neuronal Noise, Springer Series in Computational Neuroscience Vol. 8 (Springer, New York, 2012).

[11] Alex Bukoski, D. A. Steyn-Ross, and Moira L. Steyn-Ross, Channel-noise-induced critical slowing in the subthreshold Hodgkin-Huxley neuron, Phys. Rev. E 91, 032708 (2015). 
[12] Gustavo Deco, Viktor K. Jirsa, Peter A. Robinson, Michael Breakspear, and Karl Friston, The dynamic brain: from spiking neurons to neural masses and cortical fields, PLoS Comput. Biol. 4, e1000092 (2008).

[13] Javier Baladron, Diego Fasoli, Olivier Faugeras, and Jonathan Touboul, Mean-field description and propagation of chaos in networks of Hodgkin-Huxley and FitzHugh-Nagumo neurons, J. Math. Neurosci. 2, 10 (2012).

[14] Henry Markram, The blue brain project, Nat. Rev. Neurosci. 7, 153 (2006).

[15] Eric R. Kandel, Henry Markram, Paul M. Matthews, Rafael Yuste, and Christof Koch, Neuroscience thinks big (and collaboratively), Nat. Rev. Neurosci. 14, 659 (2013).

[16] H. R. Wilson and J. D. Cowan, Excitatory and inhibitory interactions in localized populations of model neurons, Biophys. J. 12, 1 (1972).

[17] V. K. Jirsa and H. Haken, A Field Theory of Electromagnetic Brain Activity, Phys. Rev. Lett. 77, 960 (1996).

[18] P. A. Robinson, C. J. Rennie, and J. J. Wright, Propagation and stability of waves of electrical activity in the cerebral cortex, Phys. Rev. E 56, 826 (1997).

[19] Moira L. Steyn-Ross, D. A. Steyn-Ross, J. W. Sleigh, and D. T. J. Liley, Theoretical electroencephalogram stationary spectrum for a white-noise-driven cortex: Evidence for a general anesthetic-induced phase transition, Phys. Rev. E 60, 7299 (1999).

[20] D. T. J. Liley, P. J. Cadusch, and J. J. Wright, A continuum theory of electro-cortical activity, Neurocomputing 26-27, 795 (1999).

[21] D. A. Steyn-Ross, M. L. Steyn-Ross, J. W. Sleigh, M. T. Wilson, I. P. Gillies, and J. J. Wright, The sleep cycle modelled as a cortical phase transition, J. Biol. Phys. 31, 547 (2005).

[22] Moira L. Steyn-Ross, D. A. Steyn-Ross, and J. W. Sleigh, Interacting Turing-Hopf Instabilities Drive Symmetry-Breaking Transitions in a Mean-Field Model of the Cortex: A Mechanism for the Slow Oscillation, Phys. Rev. X 3, 021005 (2013).

[23] M. T. Wilson, P. A. Robinson, B. O'Neill, and D. A. Steyn-Ross, Complementarity of spike- and rate-based dynamics of neural systems, PLoS Comput. Biol. 8, e1002560 (2012).

[24] N. Brunel and V. Hakim, Fast global oscillations in networks of integrate-and-fire neurons with low firing rates, Neural Comput. 11, 1621 (1999).

[25] Michael A. Buice and Jack D. Cowan, Field-theoretic approach to fluctuation effects in neural networks, Phys. Rev. E 75, 051919 (2007).

[26] Sami El Boustani and Alain Destexhe, A master equation formalism for macroscopic modeling of asynchronous irregular activity states, Neural Comput. 21, 46 (2009).

[27] Paul C. Bressloff, Stochastic neural field theory and the systemsize expansion, SIAM J. Appl. Math. 70, 1488 (2009).

[28] Michael A. Buice and Carson C. Chow, Beyond mean field theory: Statistical field theory for neural networks, J. Stat. Mech. (2013) P03003.

[29] C. W. Gardiner, Handbook of Stochastic Methods for Physics, Chemistry, and the Natural Sciences, 3rd ed., Springer Series in Synergetics Vol. 13 (Springer-Verlag, Berlin, Heidelberg, New York, 2004).

[30] K. G. Wilson and J. Kogut, The renormalization group and the $\epsilon$-expansion, Phys. Rep. 12, 75 (1974).
[31] C. W. Gardiner, Adiabatic elimination in stochastic systems. I. Formulation of methods and application to few-variable systems, Phys. Rev. A 29, 2814 (1984).

[32] C. W. Gardiner and Moira L. Steyn-Ross, Adiabatic elimination in stochastic systems. II. Application to reactiondiffusion and hydrodynamic-like systems, Phys. Rev. A 29, 2823 (1984).

[33] Moira L. Steyn-Ross and C. W. Gardiner, Adiabatic elimination in stochastic systems. III. Application to renormalisation group transformations of time-dependent Ginsburg-Landau model, Phys. Rev. A 29, 2834 (1984).

[34] P. A. Robinson, H. Wu, and J. W. Kim, Neural rate equations for bursting dynamics derived from conductance-based equations, J. Theor. Biol. 250, 663 (2008).

[35] P. A. Robinson and J. W. Kim, Spike, rate, field, and hybrid methods for treating neuronal dynamics and interactions, J. Neurosci. Methods 205, 283 (2012).

[36] H. C. Tuckwell, Introduction to Theoretical Neurobiology: Linear Cable Theory and Dendritic Structure (Cambridge University Press, Cambridge, 1988), Vol. 1.

[37] E. M. Izhikevich, Dynamical Systems in Neuroscience: The Geometry of Excitability and Bursting (MIT Press, Cambridge, MA, 2007).

[38] J. Rinzel, Excitation dynamics: Insights from simplified membrane models, Fed. Proc. 44, 2944 (1985).

[39] A. Destexhe, Z. F. Mainen, and T. J. Sejnowski, Synthesis of models for excitable membranes, synaptic transmission and neuromodulation using a common kinetic formalism, J. Comput. Neurosci. 1, 195 (1994).

[40] C. J. Rennie, J. J. Wright, and P. A. Robinson, Mechanisms for cortical electrical activity and emergence of gamma rhythm, J. Theor. Biol. 205, 17 (2000).

[41] Moira L. Steyn-Ross, D. A. Steyn-Ross, M. T. Wilson, and J. W. Sleigh, Gap junctions mediate large-scale Turing structures in a mean-field cortex driven by subcortical noise, Phys. Rev. E 76, 011916 (2007).

[42] A. Melkikh and M. Sutormina, Developing Synthetic Transport Systems (Springer, Dordrecht, 2013).

[43] L. Heginbotham and R. MacKinnon, Conduction properties of the cloned Shaker K ${ }^{+}$channel, Biophys. J. 65, 2089 (1993).

[44] M. LeMasurier, L. Heginbotham, and C. Miller, KcsA: It's a potassium channel, J. Gen. Physiol. 118, 303 (2001).

[45] D. Andreucci, D. Bellaveglia, E. N. M. Cirillo, and S. Marconi, Effect of intracellular diffusion on current-voltage curves in potassium channels, Discrete Contin. Dynam. Syst. - Ser. B 19, 1837 (2014).

[46] A. Aldo Faisal, Luc P. J. Selen, and Daniel M. Wolpert, Noise in the nervous system, Nat. Rev. Neurosci. 9, 292 (2008).

[47] H. Risken, The Fokker-Planck Equation: Methods of Solution and Applications, Springer Series in Synergetics Vol. 18 (Springer-Verlag, Berlin, 1984).

[48] Takaichi Fukuda, Toshio Kosaka, Wolf Singer, and Ralf A. W. Galuske, Gap junctions among dendrites of cortical GABAergic neurons establish a dense and widespread intercolumnar network, J. Neurosci. 26, 3434 (2006).

[49] Paul E. Hand and Boyce E. Griffith, Adaptive multiscale model for simulating cardiac conduction, Proc. Natl. Acad. Sci. USA 107, 14603 (2010).

[50] Francisco J. Martinez-Wittinghan, Caterina Sellitto, Thomas W. White, Richard T. Mathias, David Paul, and Daniel A. 
Goodenough, Lens gap junctional coupling is modulated by connexin identity and the locus of gene expression, Invest. Ophthalmol. Visual Sci. 45, 3629 (2004).

[51] Maiken Nedergaard, Bruce Ransom, and Steven A. Goldman, New roles for astrocytes: Redefining the functional architecture of the brain, Trends Neurosci. 26, 523 (2003).

[52] M. Galarreta and S. Hestrin, Electrical synapses between GABA-releasing interneurons, Nat. Rev. Neurosci. 2, 425 (2001).

[53] Yun Wang, Amey Barakat, and Hongwei Zhou, Electrotonic coupling between pyramidal neurons in the neocortex, PLoS One 5, e10253 (2010).

[54] V. B. Mountcastle, Modality and topographic properties of single neurons of cat's somatic sensory cortex, J. Neurophysiol. 20, 408 (1957).

[55] D. H. Hubel, T. N. Wiesel, and M. P. Stryker, Orientation columns in macaque monkey visual cortex demonstrated by the 2-deoxyglucose autoradiographic technique, Nature (London) 269, 328 (1977).

[56] Nuno Maçarico da Costa and Kevan A. C. Martin, Whose cortical column would that be? Front. Neuroanat. 4, 16 (2010).

[57] Jon H. Kaas, Evolution of columns, modules, and domains in the neocortex of primates, Proc. Natl. Acad. Sci. USA 109, 10655 (2012).

[58] Jonathan C. Horton and Daniel L. Adams, The cortical column: A structure without a function, Philos. Trans. R. Soc., B 360, 837 (2005).

[59] M. T. Wilson, Moira L. Steyn-Ross, D. A. Steyn-Ross, and J. W. Sleigh, Predictions and simulations of cortical dynamics during natural sleep using a continuum approach, Phys. Rev. E 72, 051910 (2005).

[60] A. J. K. Phillips and P. A. Robinson, A quantitative model of sleep-wake dynamics based on the physiology of the brainstem ascending arousal system, J. Biol. Rhythms 22, 167 (2007).

[61] B. A. Lopour, S. Tasoglu, H. E. Kirsch, J. W. Sleigh, and A. J. Szeri, A continuous mapping of sleep states through association of EEG with a mesoscale cortical model, J. Comput. Neurosci. 30, 471 (2011).

[62] Moira L. Steyn-Ross, D. A. Steyn-Ross, and J. W. Sleigh, Modelling general anaesthesia as a first-order phase transition in the cortex, Prog. Biophys. Mol. Biol. 85, 369 (2004).

[63] I. Bojak and D. T. J. Liley, Modeling the effects of anesthesia on the electroencephalogram, Phys. Rev. E 71, 041902 (2005).

[64] B. Molaee-Ardekani, L. Senhadji, M. B. Shamsollahi, B. Vosoughi-Vahdat, and E. Wodey, Brain activity modeling in general anesthesia: Enhancing local mean-field models using a slow adaptive firing rate, Phys. Rev. E 76, 041911 (2007).

[65] Axel Hutt and Andre Longtin, Effects of the anesthetic agent propofol on neural populations, Cognit. Neurodyn. 4, 37 (2010).

[66] J. W. Sleigh, Moira L. Steyn-Ross, D. A. Steyn-Ross, L. J. Voss, and M. T. Wilson, Anesthesia-induced state transitions in neuronal populations, in Suppressing the Mind: Anesthetic Modulation of Memory and Consciousness, edited by A. G.
Hudetz and R. Pearce, Springer Series in Contemporary Clinical Neuroscience (Springer, Berlin, 2010), Chap. 7, p. 139.

[67] D. T. J. Liley, B. L. Foster, and I. Bojak, Mesoscopic modeling approach to anaesthetic action on brain electrical activity, in Sleep and Anesthesia: Neural Correlates in Theory and Experiment, edited by Axel Hutt, Springer Series in Computational Neuroscience Vol. 15 (Springer, Berlin, 2011), Chap. 7, p. 139.

[68] B. L. Foster, I. Bojak, and D. T. Liley, Understanding the effects of anesthetic agents on the EEG through neural field theory, Conf. Proc. IEEE Eng. Med. Biol. Soc. 2011, 4709 (2011).

[69] D. A. Steyn-Ross, M. L. Steyn-Ross, J. W. Sleigh, and M. T. Wilson, Progress in modeling EEG effects of general anesthesia: Biphasic response and hysteresis, in Sleep and Anesthesia: Neural Correlates in Theory and Experiment, edited by Axel Hutt, Springer Series in Computational Neuroscience Vol. 15 (Springer, Berlin, 2011), Chap. 8, p. 167.

[70] Rikkert Hindriks and Michel J. A. M. van Putten, Meanfield modeling of propofol-induced changes in spontaneous EEG rhythms, NeuroImage 60, 2323 (2012).

[71] A. Hutt, The anesthetic propofol shifts the frequency of maximum spectral power in EEG during general anesthesia: Analytical insights from a linear model, Front. Comput. Neurosci. 7, 2 (2013).

[72] D. A. Steyn-Ross, Moira L. Steyn-Ross, and J. W. Sleigh, Equilibrium and nonequilibrium phase transitions in a continuum model of an anesthetized cortex, in Neural Fields: Theory and Applications, edited by P. beim Graben, S. Coombes, R. Potthast, and J. J. Wright (Springer-Verlag, Berlin, 2014), Chap. 15, p. 393.

[73] P. A. Robinson, C. J. Rennie, and D. L. Rowe, Dynamics of large-scale brain activity in normal arousal states and epileptic seizures, Phys. Rev. E 65, 041924 (2002).

[74] M. Breakspear, J. A. Roberts, J. R. Terry, S. Rodrigues, N. Mahant, and P. A. Robinson, A unifying explanation of primary generalized seizures through nonlinear brain modeling and bifurcation analysis, Cereb Cortex 16, 1296 (2005).

[75] D. T. J. Liley and I. Bojak, Understanding the transition to seizure by modeling the epileptiform activity of general anesthetic agents, J. Clin. Neurophysiol. 22, 300 (2005).

[76] M. T. Wilson, J. W. Sleigh, D. A. Steyn-Ross, and Moira L. Steyn-Ross, General anesthetic-induced seizures can be explained by a mean-field model of cortical dynamics, Anesthesiology 104, 588 (2006).

[77] Mark A. Kramer, Andrew J. Szeri, James W. Sleigh, and Heidi E. Kirsch, Mechanisms of seizure propagation in a cortical model, J. Comput. Neurosci. 22, 63 (2007).

[78] Moira L. Steyn-Ross, D. A. Steyn-Ross, and J. W. Sleigh, Gap junctions modulate seizures in a mean-field model of general anesthesia for the cortex, Cognit. Neurodyn. 6, 215 (2012).

[79] Alberto E. Pereda, Sebastian Curti, Gregory Hoge, Roger Cachope, Carmen E. Flores, and John E. Rash, Gap junctionmediated electrical transmission: Regulatory mechanisms and plasticity, Biochim. Biophys. Acta 1828, 134 (2013). 